

\section{TurbSim User's Guide: Version 1.50}

B.J. Jonkman

Revised August 26, 2009 for

TurbSim version 1.50

Prepared under Task No. WER8.2102

\section{September 2009}

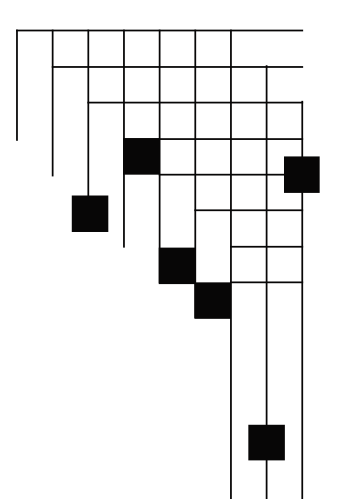

National Renewable Energy Laboratory

1617 Cole Boulevard, Golden, Colorado 80401-3393

303-275-3000 • www.nrel.gov

NREL is a national laboratory of the U.S. Department of Energy

Office of Energy Efficiency and Renewable Energy

Operated by the Alliance for Sustainable Energy, LLC

Contract No. DE-AC36-08-GO28308 


\section{NOTICE}

This report was prepared as an account of work sponsored by an agency of the United States government. Neither the United States government nor any agency thereof, nor any of their employees, makes any warranty, express or implied, or assumes any legal liability or responsibility for the accuracy, completeness, or usefulness of any information, apparatus, product, or process disclosed, or represents that its use would not infringe privately owned rights. Reference herein to any specific commercial product, process, or service by trade name, trademark, manufacturer, or otherwise does not necessarily constitute or imply its endorsement, recommendation, or favoring by the United States government or any agency thereof. The views and opinions of authors expressed herein do not necessarily state or reflect those of the United States government or any agency thereof.

Available electronically at http://www.osti.gov/bridge

Available for a processing fee to U.S. Department of Energy and its contractors, in paper, from:

U.S. Department of Energy

Office of Scientific and Technical Information

P.O. Box 62

Oak Ridge, TN 37831-0062

phone: 865.576 .8401

fax: 865.576 .5728

email: mailto:reports@adonis.osti.gov

Available for sale to the public, in paper, from:

U.S. Department of Commerce

National Technical Information Service

5285 Port Royal Road

Springfield, VA 22161

phone: 800.553.6847

fax: 703.605.6900

email: orders@ntis.fedworld.gov

online ordering: http://www.ntis.gov/ordering.htm 


\section{Acknowledgements}

TurbSim was written by Bonnie Jonkman at the National Wind Technology Center (NWTC). Funding for TurbSim came from the U.S. Department of Energy under contract No. DE-AC3608-GO28308 to NREL.

Analysis of coherent events was performed by Neil Kelley, Bonnie Jonkman, and George Scott of the National Wind Technology Center, and Professor Jan Bialasiewicz, and Lisa Redmond of the University of Colorado at Denver.

The turbulence modeling scaling parameters for the GP_LLJ and NWTCUP spectral models were developed by Neil Kelley and Bonnie Jonkman. Neil Kelley developed scaling parameters for the National Renewable Energy Laboratory wind farm models. 


\section{List of Abbreviations}

$\begin{array}{ll}\text { ART } & \text { Advanced Research Turbine } \\ \text { BLAS } & \text { Basic Linear Algebra Subprograms } \\ \text { CoRA } & \text { Colorado Research Associates } \\ \text { CTKE } & \text { coherent turbulent kinetic energy } \\ \text { CXML } & \text { Compaq Extended Math Library } \\ \text { DNS } & \text { direct numerical simulation } \\ \text { ETM } & \text { Extreme Turbulence Model } \\ \text { EWM } & \text { Extreme Wind Model } \\ \text { FF } & \text { full field } \\ \text { FFT } & \text { Fast Fourier Transform } \\ \text { FFTPACK } & \text { FFT Package } \\ \text { HH } & \text { hub height } \\ \text { IEC } & \text { International Electrotechnical Commission } \\ \text { LAPACK } & \text { Linear Algebra Package } \\ \text { LES } & \text { large-eddy simulation } \\ \text { LIST } & \text { Long-Term Inflow and Structural Testing } \\ \text { LLLJP } & \text { Lamar Low-Level Jet Project } \\ \text { NCAR } & \text { National Center for Atmospheric Research } \\ \text { NREL } & \text { National Renewable Energy Laboratory } \\ \text { NTM } & \text { Normal Turbulence Model } \\ \text { NWTC } & \text { National Wind Technology Center } \\ \text { pRNG } & \text { pseudorandom number generator } \\ \text { SONIC } & \text { sonic detection and ranging } \\ \text { TI } & \text { turbulence intensity } \\ \text { TKE } & \text { turbulent kinetic energy } \\ & \end{array}$




\section{Table of Contents}

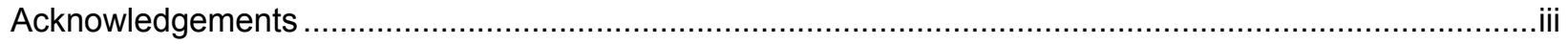

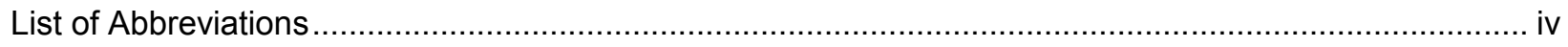

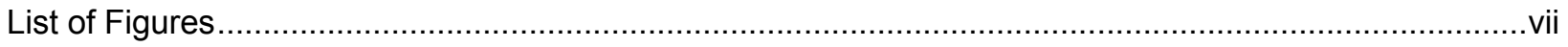

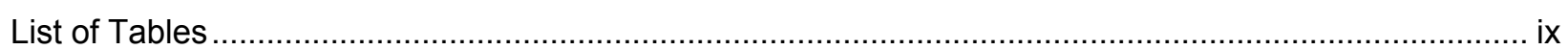

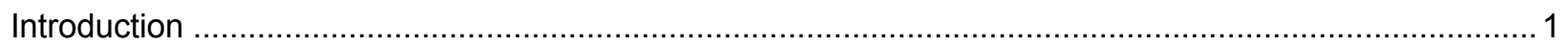

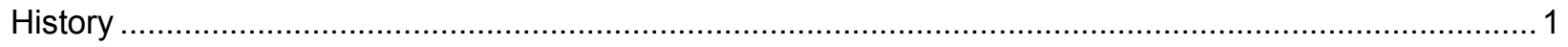

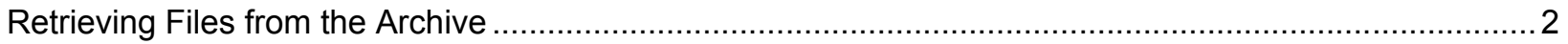

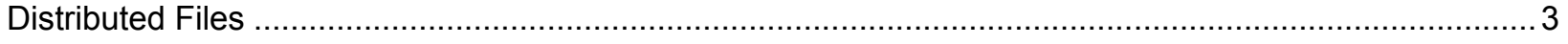

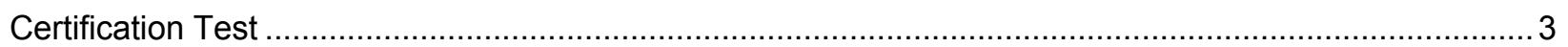

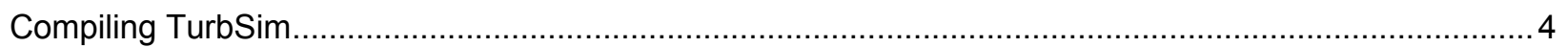

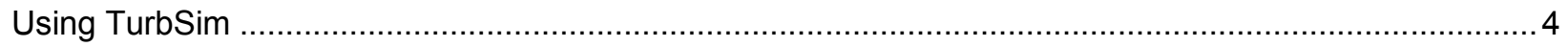

Input File

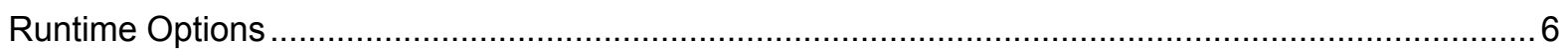

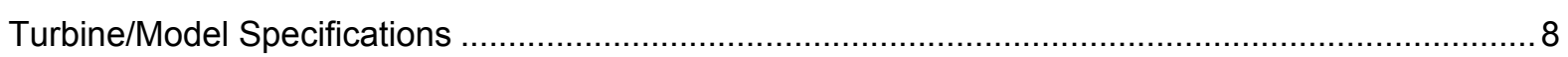

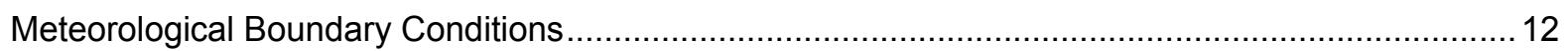

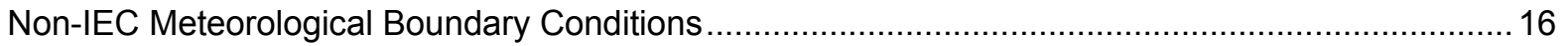

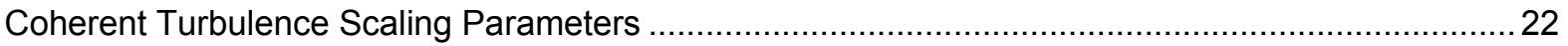

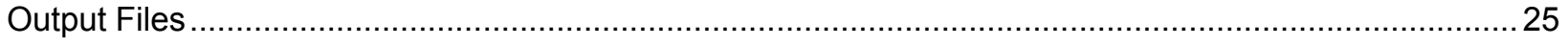

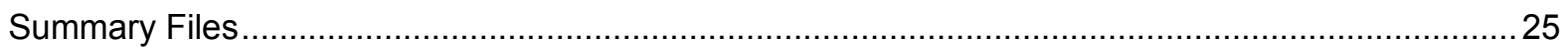

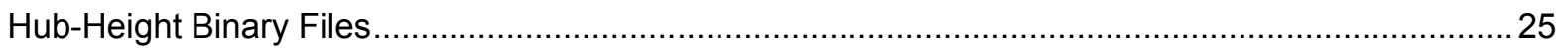

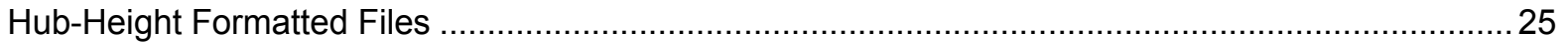

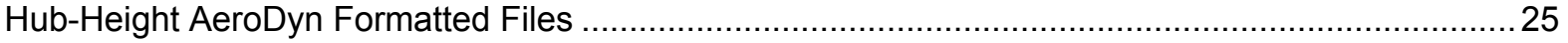

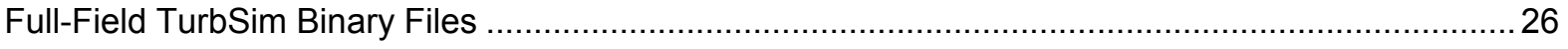

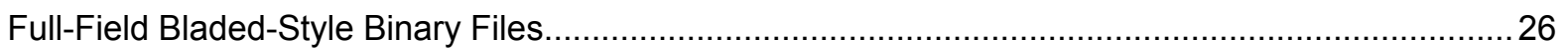

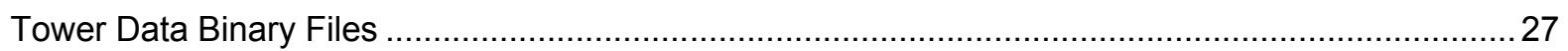

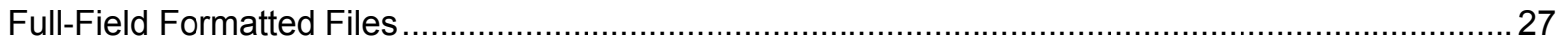

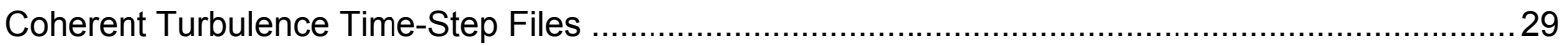

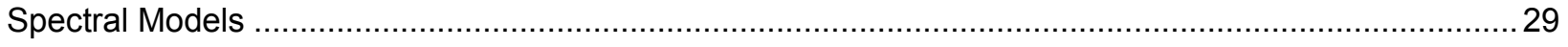

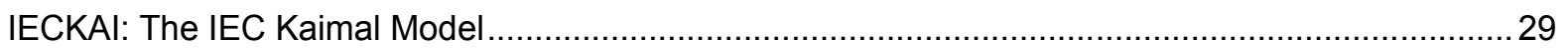

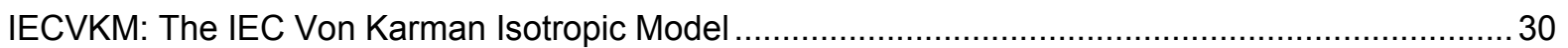

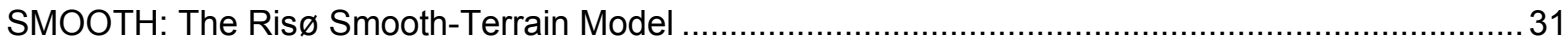

NWTCUP: The NREL National Wind Technology Center Model .................................................... 33

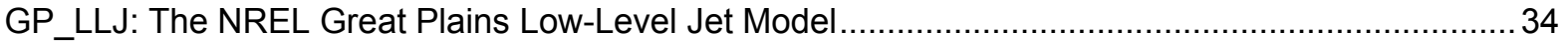

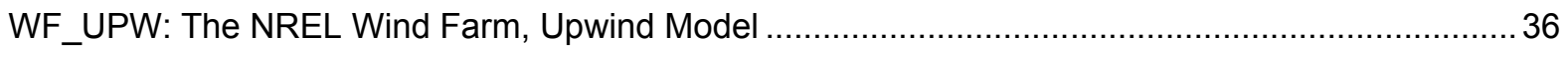

WF_14D: The NREL Wind Farm, Downwind Model (14 Rotor Diameters) ..................................... 36 
WF_07D: The NREL Wind Farm, Downwind Model (7 Rotor Diameters)..................................... 38

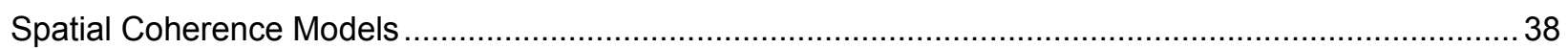

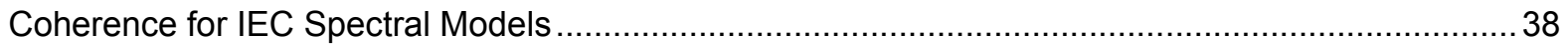

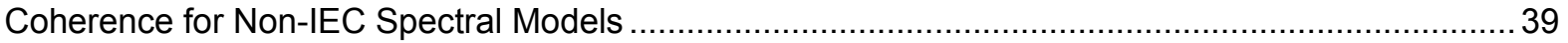

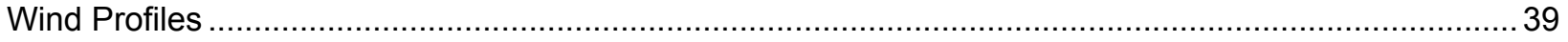

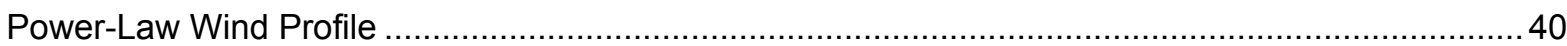

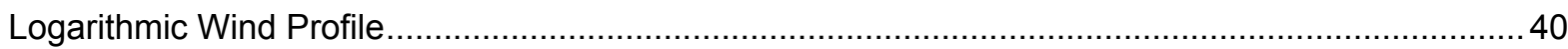

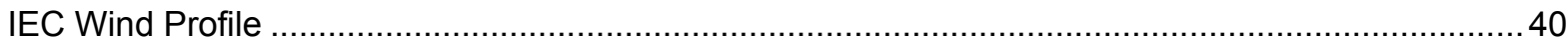

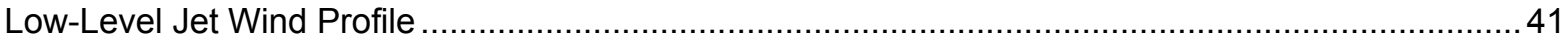

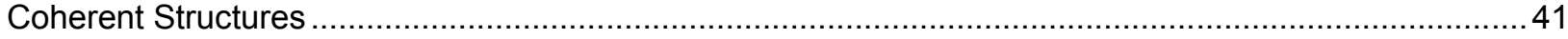

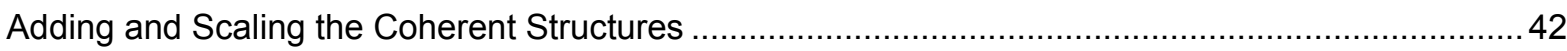

Using Coherent Turbulence Time-Step Files with AeroDyn........................................................... 44

Suggestions for Generating Coherent Turbulent Structures ............................................................. 45

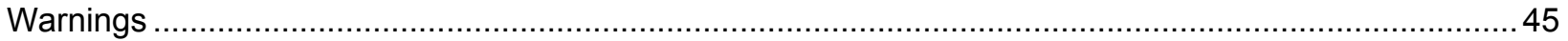

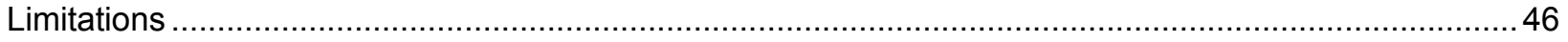

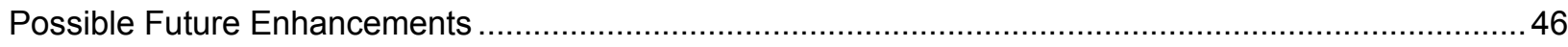

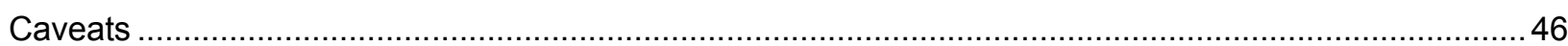

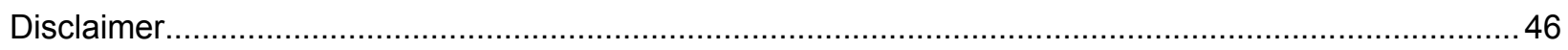

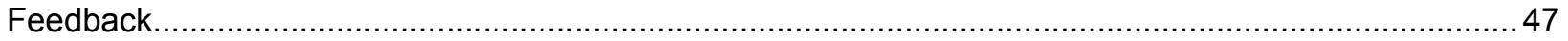

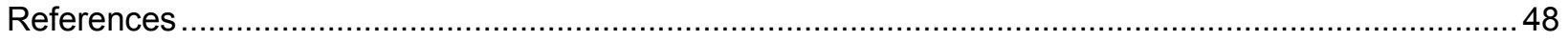

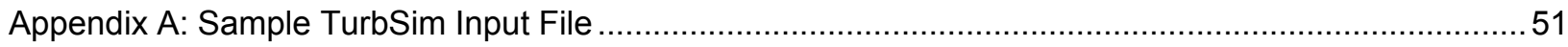

Appendix B: TurbSim Quick-Start Guidelines for IEC Turbulence ......................................................52

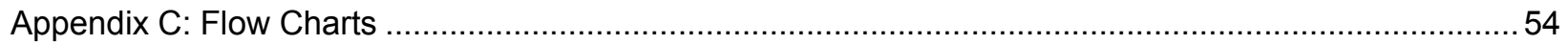

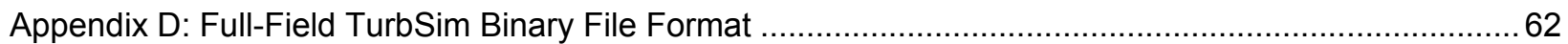

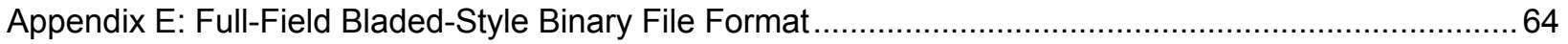

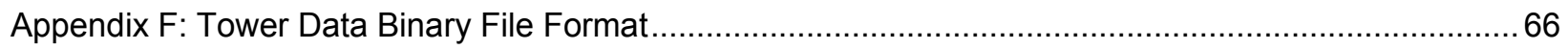

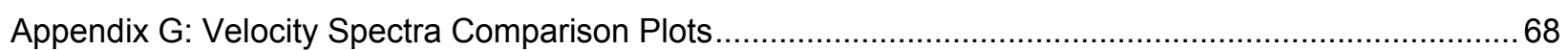

Appendix H: Sample AeroDyn Coherent Turbulence Parameter Input File ...........................................74 


\section{List of Figures}

Figure 1. TurbSim simulation method: a transformation from the frequency domain to time domain producing wind output compatible with AeroDyn; optional coherent structures are written to a separate file and superimposed in AeroDyn (they require a full-field background wind file) ................ 1

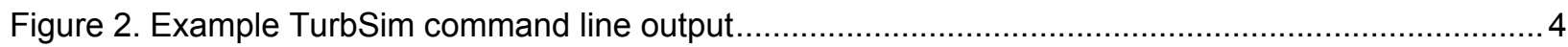

Figure 3. Coordinates of a TurbSim wind field with $15^{\circ}$ horizontal and $8^{\circ}$ vertical mean flow angles ..........5

Figure 4. Example of tower points below a rectangular grid............................................................... 7

Figure 5. Example grid and rotor placements: the circles pictured here are the rotor diameters assumed by TurbSim; the actual rotor diameter(s) will be smaller than pictured ......................................... 10

Figure 6. Example of TurbSim grids as implemented in AeroDyn: (a) The inertial frame coordinate systems and planes "marching" along positive $X$, regardless of flow angles, (b) wind field with both flow angles $0^{\circ}$, (c) the same wind field with VFlowAng $=8^{\circ}$ and HFlowAng $=15^{\circ}$

Figure 7. Longitudinal wind-speed standard deviation and TI for IEC turbulence categories as functions of

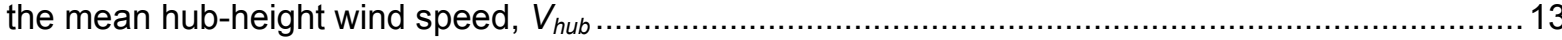

Figure 8. Coherent turbulent kinetic energy (CTKE) of an example simulation using KHTEST: the coherent structure placement in the middle of the time series is shown by the red lines....

Figure 9. Default jet wind speed for URef calculation: error bars indicate the range of random variate, $N$; dotted lines mark the tenth and ninetieth percentiles

Figure 10. Default jet height, ZJetMax, without random variates (the random variation range is approximately $\pm 50 \mathrm{~m}$ )

Figure 11. Diabatic friction velocity, $u_{*_{0}}$, normalized by URef and calculated using RefHt $=80 \mathrm{~m}$ and $\mathrm{ZO}=0.01 \mathrm{~m}$

Figure 12. Default UStar as a function of diabatic friction velocity, $u_{*_{0}}$ (left: SMOOTH, GP_LLJ, and NWTCUP models, right: wind farm models).

Figure 13. Default $u$-component coherence parameters, IncDec1, ( $a_{u}$ left, $b_{u}$ right) as a function of wind speed, using RICH_NO $=0$ and $H u b H t=80 \mathrm{~m}$ (IEC values are plotted for comparison)

Figure 14. Default $v$-component coherence parameters, IncDec2, ( $a_{v}$ left, $b_{v}$ right) as a function of wind speed, using RICH_NO=0 and $\mathrm{HubHt}=80 \mathrm{~m}$ (IEC does not define $v$-component coherence parameters)

Figure 15. Default $w$-component coherence parameters, IncDec3, ( $a_{w}$ left, $b_{w}$ right) as a function of wind speed, using RICH_NO=O and $\mathrm{HubHt}=80 \mathrm{~m}$ (IEC does not define $w$-component coherence parameters)

Figure 16. Coherent structure scaling (looking downwind): CTLy is the tower location, CTLz is the hub (height) location, and DistScl determines the size $(\mathrm{m})$ relative to the rotor disk

Figure 17. Example TurbSim FF and $\mathrm{HH}$ wind files as implemented in AeroDyn.

Figure 18. SMOOTH-model stable/neutral turbulence as a function of $R I C H \_N O$ : left: standard deviation normalized by UStar, right: Relationships between components' standard deviations.

Figure 19. NWTCUP-model stable/neutral turbulence as a function of $\mathrm{RICH} N \mathrm{NO}$ : left: standard deviation normalized by UStar, right: relationships between components' standard deviations

Figure 20. GPLLJ-model stable/neutral turbulence as a function of local stability and shear velocities .... 35 
Figure 21. WF_UPW-model stable/neutral turbulence as a function of $R I C H \_N O$ : left: standard deviation normalized by UStar, right: relationships between components' standard deviations

Figure 22. WF_07D- and WF_14D-model stable/neutral turbulence as a function of RICH_NO: left: standard deviation normalized by UStar, right: ratios of standard deviations ....

Figure 23. Example wind-speed profiles generated in TurbSim for the GP_LLJ model using a 90-m hub-

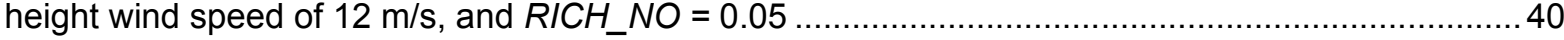

Figure 24. Example jet wind profiles with a $12 \mathrm{~m} / \mathrm{s}$ wind speed at $80 \mathrm{~m}$ and $\mathrm{R} / \mathrm{CH}$ _NO $=0.05 \ldots \ldots \ldots \ldots . . . .41$

Figure 25. Coherent structures in a 10-minute period from the NWTC LIST dataset: the time series shows the 3-s mean CTKE (solid black line) crossing the dashed threshold line, which indicates the location of the coherent structures (indicated in blue).

Figure 26. Example time series from the NWTCUP model: the black line indicates the background wind file; the green shows the addition of events in a coherent time-step file (".cts") ................................ 43

Figure A-1. Sample TurbSim input file

Figure B-1. Sample TurbSim input file for IEC turbulence: parameters shown in blue should be changed based on the turbine configuration; parameters shown in red should be changed for each load case and simulation.

Figure C-1. Overview of the TurbSim simulation method; blue lines indicate processes influenced by input-file parameters; black lines indicate internal variables and processes ...................................5 54

Figure C-2. Parameters in the Runtime Options section of the input file ................................................ 55

Figure C-3. Parameters in the Turbine/Model Specifications section of the TurbSim input file ................56

Figure C-4. Parameters in the Meteorological Boundary Conditions section of the TurbSim input file (for

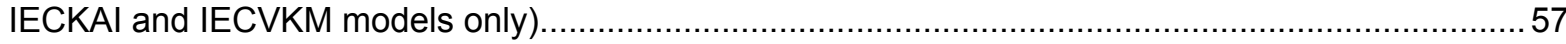

Figure C-5. Parameters in the Meteorological Boundary Conditions section of the TurbSim input file (for models other than IECKAI and IECVKM).

Figure C-6. Parameters in the Non-IEC Meteorological Boundary Conditions section of the TurbSim input file

Figure C-7. Default input values for the for the Meteorological Boundary Conditions and Non-IEC Meteorological Boundary Conditions sections of the TurbSim input file

Figure C-8. Parameters for coherent structures and the Coherent Turbulence Scaling Parameters section of the TurbSim input file; the SMOOTH model uses the GP_LLJ scaling....

Figure G-1. Neutral velocity spectra for the 8 spectral models available in TurbSim, using a $15 \mathrm{~m} / \mathrm{s}$ wind speed at $80 \mathrm{~m}$; IECKAI and IECVKM use NTM category "B" and 61400-1 $3^{\text {rd }}$ ed. scaling; the non-IEC models use RICH_NO $=0$ and UStar = "default"....

Figure G-2. Neutral velocity spectra for the 8 spectral models available in TurbSim, using a $15 \mathrm{~m} / \mathrm{s}$ wind speed at $80 \mathrm{~m}$; IECKAI and IECVKM use NTM category "B" and 61400-1 $3^{\text {rd }}$ ed. scaling; the non-IEC models use RICH_NO $=0$ and UStar $=1.1 \mathrm{~m} / \mathrm{s}$.

Figure G-3. Stable velocity spectra using a $15 \mathrm{~m} / \mathrm{s}$ wind speed at $80 \mathrm{~m}$; the non-IEC models use RICH_NO $=0.05$ and UStar = "default"; The IEC models, which are neutral $\left(R I C H \_N O=0\right)$, were added for reference; they use NTM category "B" and 61400-1 $3^{\text {rd }}$ ed. scaling .....

Figure G-4. Stable velocity spectra using a $15 \mathrm{~m} / \mathrm{s}$ wind speed at $80 \mathrm{~m}$; the non-IEC models use $\mathrm{RICH} \mathrm{NO}=0.05$ and UStar $=1.1 \mathrm{~m} / \mathrm{s}$; the IEC models, which are neutral $(\mathrm{RICH} N \mathrm{NO}=0)$, were added for reference; they use NTM category "B" and 61400-1 $3^{\text {rd }}$ ed. scaling .....

Figure G-5. Unstable velocity spectra using a $15 \mathrm{~m} / \mathrm{s}$ wind speed at $80 \mathrm{~m}$; the non-IEC models use $R I C H \_N O=-0.05$ and UStar = "default"; the IEC models, which are neutral $\left(R I C H \_N O=0\right)$, were added for reference; they use NTM category "B" and 61400-1 $3^{\text {rd }}$ ed. scaling ..... 
Figure G-6. Unstable velocity spectra using a $15 \mathrm{~m} / \mathrm{s}$ wind speed at $80 \mathrm{~m}$; the non-IEC models use $R I C H \_N O=-0.05$ and $U S t a r=1.1 \mathrm{~m} / \mathrm{s}$; the IEC models, which are neutral $\left(R / C H \_N O=0\right)$, were added for reference; they use NTM category "B" and 61400-1 $3^{\text {rd }}$ ed. scaling .......

Figure H-1. Sample AeroDyn coherent turbulence parameter input file.....

\section{List of Tables}

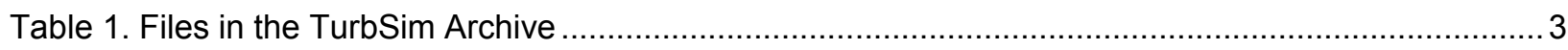

Table 2. Definitions of TurbSim Wind-Component Coordinate Systems ............................................. 5

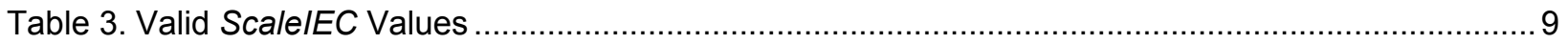

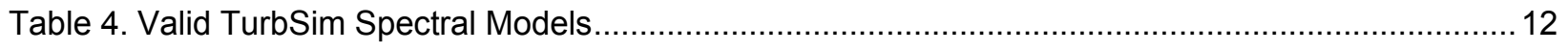

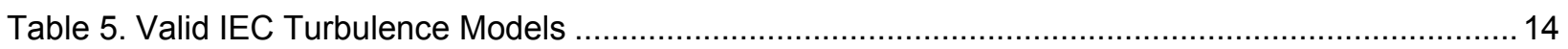

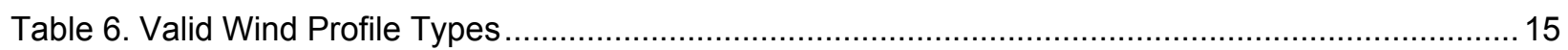

Table 7. Default Inputs for Meteorological Boundary Conditions......................................................... 17

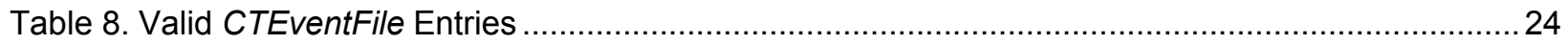

Table 9. Parameters in Hub-Height Binary and Formatted Files ....................................................... 26

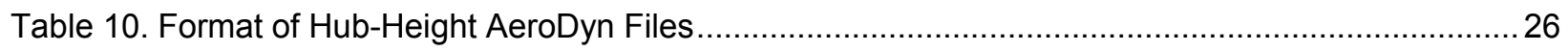

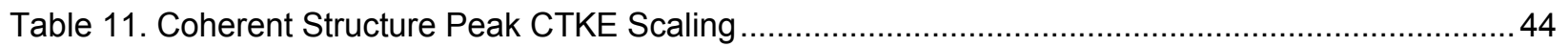

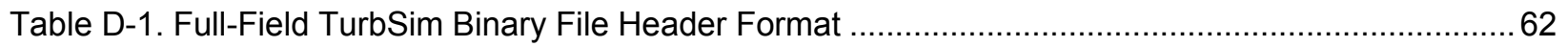

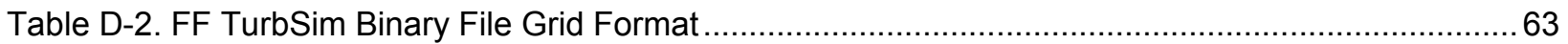

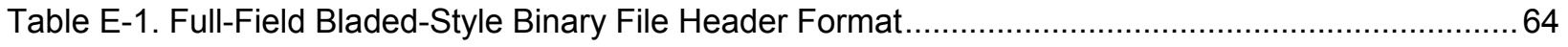

Table E-2. Format of Grid Velocities in Full-Field Bladed-Style Binary File Format .................................65

Table F-1: Format of Header in TurbSim Binary Tower-Data File ........................................................... 66

Table F-2: Format of Grid Velocities in TurbSim Binary Tower-Data File ............................................ 66 


\section{Introduction}

TurbSim is a stochastic, full-field, turbulent-wind simulator. It uses a statistical model (as opposed to a physics-based model) to numerically simulate time series of three-component windspeed vectors at points in a two-dimensional vertical rectangular grid that is fixed in space. TurbSim output can be used as input into AeroDyn-based [1] codes such as FAST [2], YawDyn [3], or MSC.ADAMS ${ }^{\circledR}$ [4]. AeroDyn uses Taylor's frozen turbulence hypothesis to obtain local wind speeds, interpolating the TurbSim-generated fields in both time and space.

Spectra of velocity components and spatial coherence are defined in the frequency domain, and an inverse Fourier transform produces time series. The underlying theory behind this method of simulating time series assumes a stationary process. To simulate non-stationary components, TurbSim - used with AeroDyn - can superimpose coherent turbulent structures onto the time series it generates. The basic simulation method is summarized in Figure 1.

\section{History}

In 1988, Paul Veers of Sandia National Laboratories wrote a program called SNLWIND [5] that could generate full-field turbulent wind for the streamwise $(u)$ component only. In 1992, Neil Kelley of the National Renewable Energy Laboratory (NREL) added several spectral models to SNLWIND and modified it to generate the $v$ and $w$ components [6]. SNLWIND-3D was the result. During the next five years NREL researchers modified the program further, including adding the Kaimal and von Karman spectral models specified by the International Electrotechnical Commission (IEC). Gary Desroachers modified it to run on many different platforms by including C-preprocessor directives for conditional compilation. In 1997, Marshall

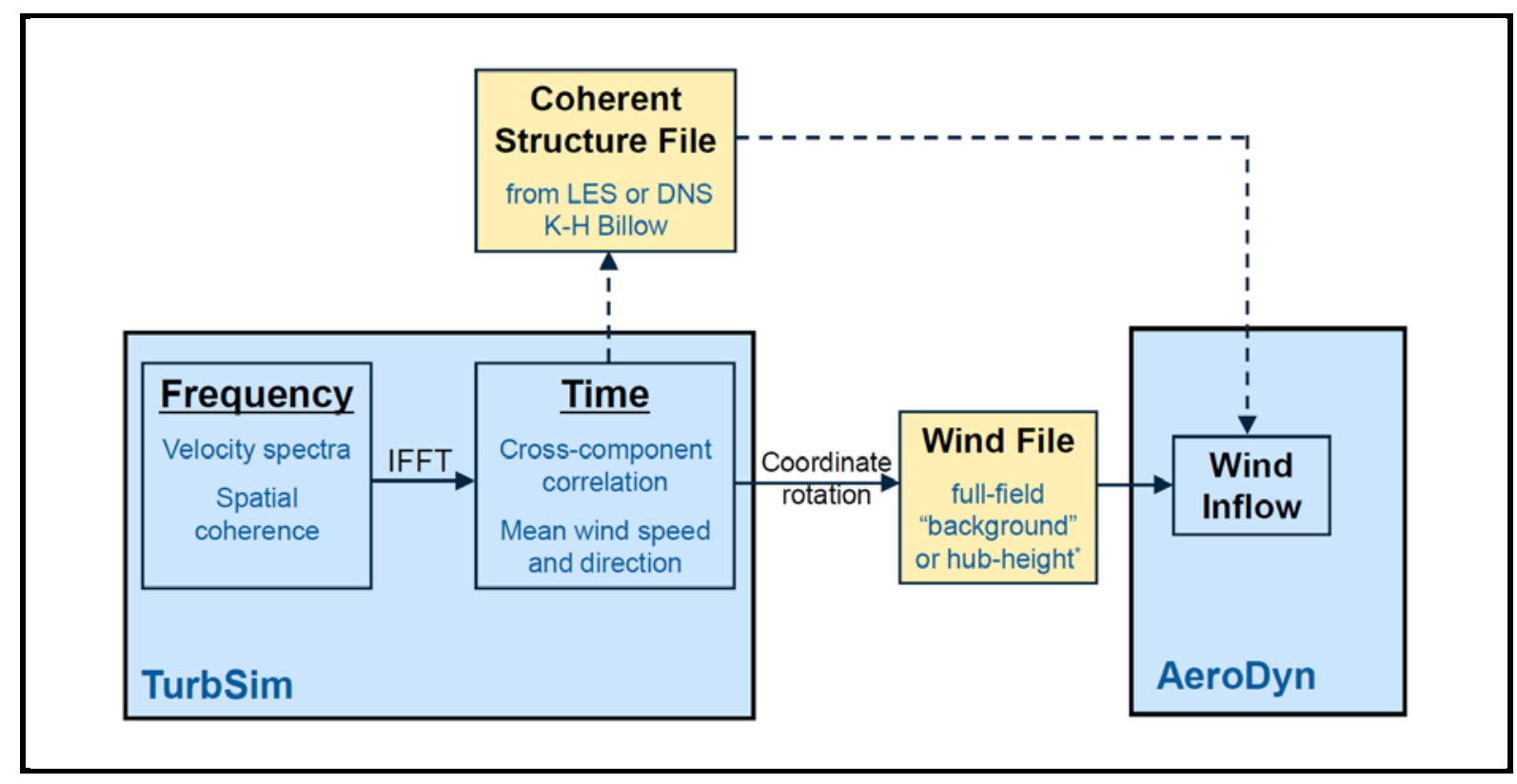

Figure 1. TurbSim simulation method: a transformation from the frequency domain to time domain producing wind output compatible with AeroDyn; optional coherent structures are written to a separate file and superimposed in AeroDyn (they require a full-field background wind file) 
Buhl added the ability to generate binary files that are compatible with Garrad Hassan's "GH Bladed" turbine design code [7].

Both SNLWIND and SNLWIND-3D were written in FORTRAN 77 and required recompilation for different grid densities and run lengths. This made using and supporting the programs difficult.

In January 2000, Neil Kelley removed all of the spectral models except the two defined by the IEC, and Marshall Buhl modernized the remaining code by rewriting all but the Fast Fourier Transform (FFT) routines in Fortran 95 and eliminating the C-preprocessor directives. Buhl's changes included using dynamic-memory allocation for the big arrays, which eliminated the need to recompile for different grid sizes and run lengths. He modified the input and output file format, streamlined processes, and added the ability to generate hub-height files in AeroDyn format. Because the changes were substantial, Buhl renamed the program SNwind (Sandia/NREL Wind) [8].

In 2003, NREL researchers updated the code to add results from the Lamar Low-Level Jet Project (LLLJP) and from the National Wind Technology Center (NWTC) Long-Term Inflow and Structural Testing (LIST) project. Bonnie Jonkman also added the spectral models from SNLWIND-3D that were removed from SNwind, and she replaced the FORTRAN 77 FFT routines with more modern routines from the Compaq Extended Math Library (CXML). Jonkman made changes to the Cholesky factorization algorithm, which sped up the code and allowed for a significant reduction in the memory required to run the program. She eliminated the requirement that the grid be an even number of points in each direction, and allowed the grid height to be different from its width. After these enhancements were in place, the code was modified to generate coherent structures with realistic temporal and spatial scaling, and coherent turbulence time-step files became another output option. The code was then renamed TurbSim (turbulence simulator) because of its ability to generate coherent turbulence.

In 2005, Bonnie Jonkman replaced the CXML FFT routines with routines from FFTPACK [9] so that TurbSim could be compiled on the Intel ${ }^{\circledR}$ Visual Fortran compiler. This made the code run much faster and also made it more portable. In 2009, Jonkman updated algorithms in the code to enable users to create much larger grids than were possible in earlier versions.

Neil Kelley has written a companion document, Overview of the TurbSim Stochastic Inflow Turbulence Simulator [10], which discusses the development of TurbSim and includes some of the theory behind that development.

\section{Retrieving Files from the Archive}

The TurbSim archive can be downloaded from the NREL Web server page at http://wind.nrel.gov/designcodes/. The downloaded file will have a name like "TurbSim_v150.exe." Create a TurbSim folder somewhere on your file system and put this file there. You can double click on it from Windows Explorer or type "TurbSim_v150" (or the exact file name) at a command prompt, using the TurbSim folder as the current directory. Running this executable file creates some files and folders. Please see Marshall Buhl's paper Installing NWTC Design Codes [11] for information on how to set up TurbSim to run in any folder. 
To be able to generate coherent structures with TurbSim, users will also need to download the coherent structures archive from NREL's Web server page. The file is named

"TSM_structures.exe." Create a folder on your file system and put this file there. Execute the program by double clicking on it or by typing "TSM_structures" at a command prompt with the folder you created as the current directory. When executed, this archive creates the files and folders used to define coherent structures. It is necessary to type the name of the path to these folders in TurbSim and AeroDyn input files.

\section{Distributed Files}

The archive contains the TurbSim executable program for both 32- and 64-bit Windows ${ }^{\circledR}$ platforms. See Table 1 for a complete list of the files included in the TurbSim archive.

\section{Certification Test}

Before using TurbSim for the first time, run the certification testing program. It is a batch file called "CertTest.bat" and is located in the "Test" folder. To test the installation, edit "CertTest.bat" and set the environment variables found near the top of the file to settings that are compatible with your system. You probably will have to change only the "Editor" variable. Then open a command window, go to the Test folder, and type "CertTest" or - if you have

MATLAB ${ }^{\circledR}[12]$ installed on your computer and would like to see plots of the data-type "CertTest MATLAB."

Table 1. Files in the TurbSim Archive

\begin{tabular}{|c|c|}
\hline File(s) & Description \\
\hline ArcFiles.txt & The list of files that are written to the archive \\
\hline Archive.bat & The batch file that creates the archive \\
\hline ChangeLog.txt & The list of changes to TurbSim \\
\hline Disclaimer.txt & The software disclaimer \\
\hline RunTurbSim.pl & A sample Perl script used to run TurbSim, using a different seed each time \\
\hline TurbSim.exe & The TurbSim program for 32 -bit Windows $®$ platforms \\
\hline TurbSim64.exe & The TurbSim program for 64 -bit Windows $®$ platforms \\
\hline TurbSim.inp & A sample input file \\
\hline TurbSim.pdf & The user's guide in PDF format \\
\hline TurbSim_AD.ctp & A sample AeroDyn coherent turbulence parameter input file \\
\hline TurbSimOverview.pdf & Kelley \& Jonkman's overview of TurbSim, in PDF format [10] \\
\hline Sourcel** & The Fortran source code for TurbSim \\
\hline Testl** & $\begin{array}{l}\text { Files used to run and manage the certification tests and MATLAB® scripts for } \\
\text { reading TurbSim data }\end{array}$ \\
\hline TestlEventDatal*.* & Files used to run the certification tests with coherent turbulence \\
\hline Test|TstFiles $\left.\right|^{*}{ }^{*}$ & NREL results for the certification tests \\
\hline
\end{tabular}


When the certification testing program is run, TurbSim executes several times. The test procedure compares the new results to those stored in the "TestlTstFiles" folder, and it writes the differences between the output files to a file called "CertTest.out." If you have specified the "MATLAB" option, MATLAB opens and plots many results. It might be necessary to close the MATLAB program before the test procedure can continue. Before finishing, the test procedure automatically opens the "CertTest.out" file with the editor you specified with the "Editor" variable. Scan through the file; the only differences should be the date and time stamps in the headers of the files and the CPU time in the summary files. If you recompiled TurbSim with another compiler, some slight differences could appear in the last digit of many of the numbers.

\section{Compiling TurbSim}

It should not be necessary to compile TurbSim unless you want to make changes to the code or want to run TurbSim using a different operating system. The archive contains Fortran code specific to TurbSim. It also contains the Fortran FFTPACK version 4.1 [9], LAPACK version 3.0 [13], BLAS [14], [15], and RanLux [16] routines that TurbSim uses. Users must also download the NWTC Subroutine Library version 1.01.09, [17], which TurbSim uses. It can be found under Miscellaneous Software on the NREL Web server page at http://wind.nrel.gov/designcodes/.

The code has been written primarily for the Intel® Visual Fortran compiler. To port TurbSim to another platform or compiler, it might be necessary to make changes in the NWTC Subroutine Library's SysVF.f90 file and possibly the BLAS LSAME() function. If you have access to an optimized BLAS library, you are encouraged to link your code with it instead of using the reference BLAS routines included in the TurbSim archive.

\section{Using TurbSim}

To begin using TurbSim, a text input file is required. (Sample input files-which can be modified - are contained in the TurbSim archive and in Appendix A.) Next, to run TurbSim enter "turbsim $[/ \mathrm{h}]$ $[<$ RootName.ext $>$ ]" at a command prompt; /h and $<$ RootName.ext $>$ are optional. The $/ \mathrm{h}$ switch generates a help message, and $<$ RootName.ext $>$ is the name of the TurbSim input file. Following are two examples:

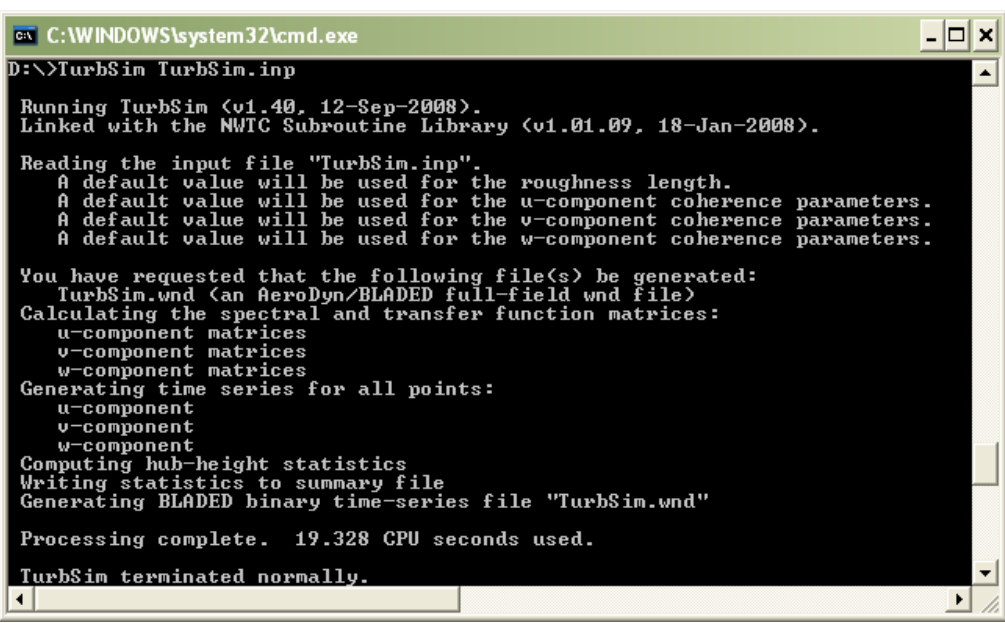

Figure 2. Example TurbSim command line output 
- turbsim

This starts TurbSim and opens the input file "turbsim.inp." It is equivalent to entering

"turbsim turbsim.inp."

- turbsim myroot.tsm

This starts TurbSim and opens the input file "myroot.tsm."

All output files have the specified root file name and different extensions.

Wind components are defined in two separate coordinate systems as described in Table 2 and pictured in Figure 3. TurbSim computes winds in a coordinate system aligned with the direction of the mean velocity vector at each point in space. The velocities are rotated to the inertial reference frame coordinate system before they are written to output files. A quick-start guide for using the most basic turbulence is included in Appendix B.

\section{Input File}

TurbSim reads a text input file to set the parameters required for the program to execute. The parameters are divided into several sections (discussed below). The heading for each parameter contains its name, a description, and the units for that parameter. The names of the parameters are provided for reference, but TurbSim does not read those names from the input file. Note that other programs or scripts that generate TurbSim input files, however, can and do use these parameter names.

TurbSim assumes that parameters are located on specific lines, so do not add or remove lines from the sample input files included in the archive. None of the parameters are case sensitive. The text of an example TurbSim input file is included in Appendix A of this guide.

Table 2. Definitions of TurbSim WindComponent Coordinate Systems

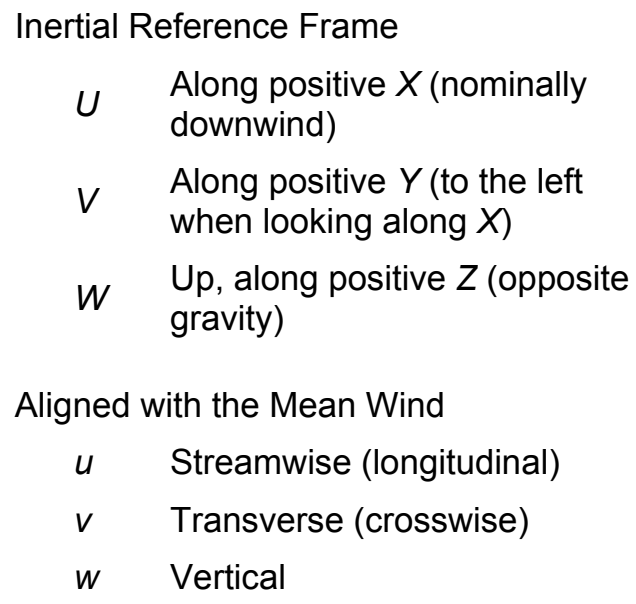

W Up, along positive $Z$ (opposite gravity)

Aligned with the Mean Wind

$u \quad$ Streamwise (longitudinal)

$v \quad$ Transverse (crosswise)

w Vertical

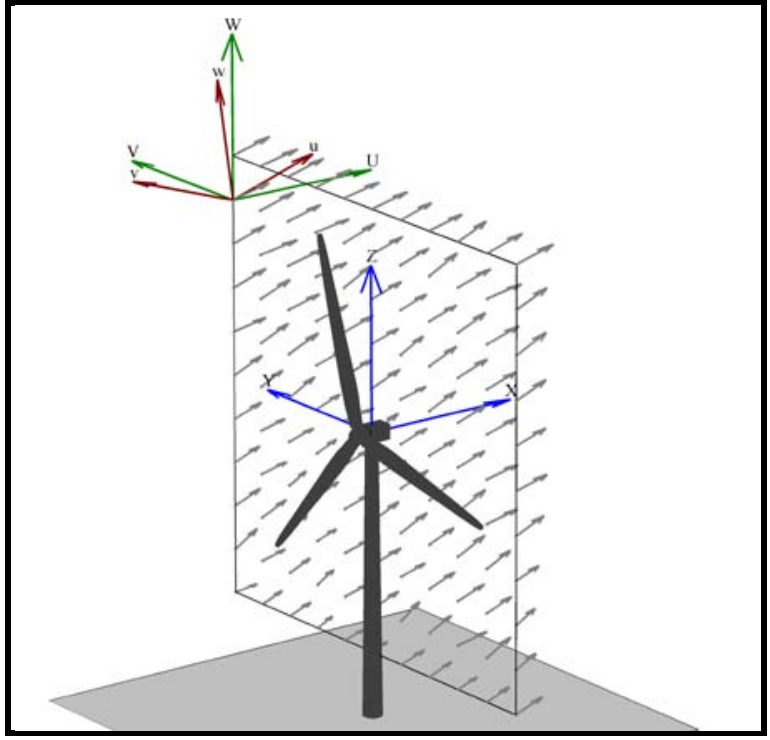

Figure 3. Coordinates of a TurbSim wind field with $15^{\circ}$ horizontal and $8^{\circ}$ vertical mean flow angles 


\section{Runtime Options}

The Runtime Options section initializes the pseudorandom number generator (pRNG) and tells TurbSim what type of output to generate. Appendix C contains a flow chart showing the function of the input parameters from this section. Users can choose any combination of output types listed in this section, but at least one output file must be generated to successfully run the code. The Output Files section of this guide provides more complete descriptions of these file types.

\section{RandSeed1: The First Random Seed [-]}

This input parameter is used in conjunction with the next parameter, RandSeed2; it tells TurbSim how to initialize the pRNG. This random seed must be an integer between -2147483648 and 2147483647 (inclusive).

The random numbers generated by the pRNG are used to create random phases (one per frequency per grid point per wind component) for the velocity time series. When the pRNG is initialized in the same way (i.e., RandSeed 1 and RandSeed 2 are not changed), the user can reproduce the same random phases between runs, which is useful in comparing the effects of changes to other input parameters. Random numbers also are used to generate some default input values and the superimposed coherent structures for the non-IEC spectral models.

\section{RandSeed2: The Second Random Seed or pRNG to Use [-]}

This input parameter indicates which of three available pRNGs to use. This value is a second random seed or the strings "RNSNLW" or "RanLux." Using RanLux is recommended because initial tests show that it seems to be the best behaved of the three generators.

If RandSeed 2 is a random seed, it must be an integer between -2147483648 and 2147483647 (inclusive). TurbSim then uses the two seeds to initialize the intrinsic pRNG-which uses two separate congruential generators together to produce a period of about $10^{18}$. This intrinsic pRNG is based on an algorithm developed by Pierre L'ecuyer [18] and is identical to the one found in SNwind.

If RandSeed 2 is the string "RNSNLW," TurbSim generates random numbers using the algorithm found in SNLWIND and SNLWIND-3D. It is initialized with only one seed.

If RandSeed 2 is the string "RanLux," TurbSim uses Lüscher's level 3 "Luxury Pseudorandom Numbers" [19], [20]. This pRNG is based on a subtract-and-borrow algorithm with a period on the order of about $10^{171}$ and is modified by throwing numbers away to destroy correlations. This pRNG is initialized with only one seed.

\section{WrBHHTP: Write Binary Hub-Height Turbulence Parameters? [T/F]}

This parameter must be either "true" or "false." Setting this output option to "true" generates a binary file with a ".bin" extension. The file contains time series of wind data and turbulence parameters from the center grid point at hub height $(\mathrm{HH})$.

\section{WrFHHTP: Write Formatted Hub-Height Turbulence Parameters? [T/F]}

This "true" or "false" parameter is similar to the previous parameter, WrBHHTP. When set to "true," it generates a file containing time series of wind data and turbulence parameters from the center grid point at hub height. This file is formatted (i.e., human-readable text) and has a ".dat" extension. 


\section{WrADHH: Write AeroDyn hub-height files? [T/F]}

This "true" or "false" parameter provides an option to generate time series in the AeroDyn hubheight format. These files have an ".hh" extension.

\section{WrADFF: Write AeroDyn Full-Field Files? [T/F]}

This parameter must be either "true" or "false." It provides the option to generate binary, fullfield (FF) time series in a format designed to be read by AeroDyn. This format is unique to TurbSim and provides an encoding scheme designed to give maximum resolution. It contains all the information necessary to decode the time series in one file, but it is not compatible with $\mathrm{GH}$ Bladed. This file format will be supported in AeroDyn v12.60 and later versions. Continue to use the Bladed-compatible FF files (parameter $W r B L F F$ ) with all other AeroDyn versions. These files have a ".bts" extension.

\section{WrBLFF: Write Bladed-Style Full-Field Files? [T/F]}

Like the $W r A D F F$ parameter, the $W r B L F F$ "true" or "false" parameter also provides the option to generate binary, full-field (FF) time series in a format designed to be read by AeroDyn. This format is compatible with GH Bladed, but in some cases the encoding scheme provides less resolution than the files generated by $W r A D F F$. These files have a ".wnd" extension.

\section{WrADTWR: Generate AeroDyn Tower Points? [T/F]}

The WrADTWR "true" or "false" parameter determines whether TurbSim generates binary tower time series, which contain points in a line at the tower centerline from the bottom of the rectangular grid to the ground. Figure 4 shows an example of the location of tower points. If a user chooses to output FF time series in the format unique to TurbSim using WrADFF (".bts" files), these tower points are added to the FF binary output file. Otherwise, a separate towerpoints binary file is created using an encoding scheme similar to the Bladed-style FF file format used in the ".wnd" files (generated with parameter $W R B L F F$ ). This extra binary file for the tower points has a ".twr" extension.

\section{WrFMTFF: Write Formatted Full-Field Files? [T/F]}

This "true" or "false" output option determines whether TurbSim generates FF time series in SNLWIND-3D human-readable format. One file is generated for each wind component, and the three files have extensions ".u," ".v," and ".w," respectively. Please note that AeroDyn cannot read these text files.

\section{WrACT: Write Coherent Turbulence Files? [T/F]}

This "true" or "false" parameter indicates whether coherent turbulence should be generated, creating time-step files in AeroDyn format. The coherent turbulence feature works only with non-IEC spectral models when the gradient Richardson number (input parameter $\mathrm{RICH}_{-} \mathrm{NO}$ ) is greater than -0.05 . The mean wind speed at the top of the coherent structure also must be greater than the mean wind speed at the bottom of the coherent structure. The Coherent Turbulence Scaling Parameters section of the input file discusses how to set the coherent structure location.

The coherent turbulence time-step files, which have a ".cts" extension, are intended to be superimposed on background FF

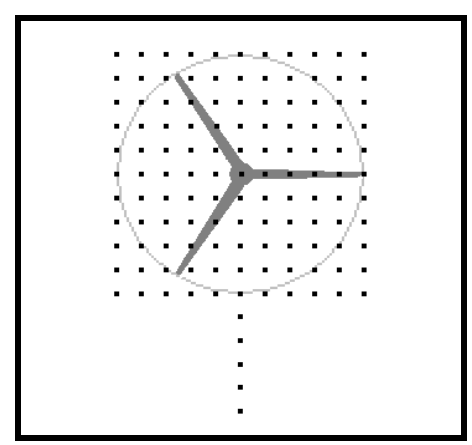

Figure 4. Example of tower points below a rectangular grid 
turbulence files. As a result, TurbSim also creates binary FF time series ( $W r B L F F$ or $W r A D F F)$ when a coherent turbulence time-step file is requested. If no FF time series format has been specified, TurbSim creates a GH Bladed-style binary FF file ( $W r B L F F$ “.wnd" file). For more information on using these ".cts" files, see the Using Coherent Turbulence Time-Step Files with AeroDyn section in this guide.

\section{Clockwise: Does the Turbine Rotate Clockwise? [T/F]}

This true" or "false" parameter is a flag to indicate whether the turbine rotates in a clockwise direction when looking downwind. This feature determines the order in which the horizontal grid points of the Bladed-style FF files are written (the parameter $W r B L F F$ must be "true"). Because AeroDyn also reads the Bladed-style FF files based on the direction of rotation, this flag does not affect the results when used with AeroDyn. This parameter probably is useful only for comparing FF results between Bladed and AeroDyn.

\section{ScaleIEC: Scale IEC Turbulence to Exact Standard Deviations? [0, 1, or 2]}

The ScaleIEC parameter is a switch to tell how to scale the time-domain velocity output of the IEC spectral models and is applicable to only the IECKAI and IECVKM spectral models. For numerical reasons, the turbulence intensity (TI) of the IEC spectral models-without this scaling - usually is slightly less than the specified value. Increasing the time series length and/or decreasing the size of the time step results in values closer to the specified TI. Different random seeds produce a Gaussian distribution of TI in the longitudinal wind component, due to the spatial coherence. To get the exact specified value of TI, the time series are multiplied by a scaling factor determined by the ratio of the target to the actual calculated standard deviation.

When ScaleIEC is set to " 0 ," no scaling takes place in the time domain. The result is the variation in TI discussed above. When the ScaleIEC switch has a value of "1," the time series at each simulated point use the same scaling factor with a different factor for each wind component. Those three scaling factors (one each for $u, v$, and $w$ ) are determined so that the standard deviations in wind speed (and thus TI) at the hub point are the exact value specified for the AnalysisTime-length time series that is generated. The TI at the other simulated points will vary. When ScaleIEC is " 2 ," the time series at each simulated point in space is scaled independently (i.e., each point and each component has its own scaling factor) so that the TI is the exact specified value at each point. This scaling method alters the coherence between points. Table 3 summarizes the valid input values.

\section{Turbine/Model Specifications}

The Turbine/Model Specifications section of the TurbSim input file determines the size and shape of the grid where time series is generated. It also determines the time/frequency content of the resulting time series and sets the mean flow angles. Appendix $\mathrm{C}$ contains a flow chart showing the function of the input parameters from this section.

\section{NumGrid_Z: Number of Vertical Grid Points [-]}

This input parameter is the number of grid points to generate in the vertical direction. It must be an integer greater than 1 . Unlike SNwind, which accepted only even numbers, TurbSim allows both even and odd grid-point sizes. TurbSim always generates a point at the hub, regardless of where the other grid points are located. (Note that this "extra" hub point is not contained the in binary FF files generated when $W r B L F F$ or $W r A D F F$ are set.) 
Table 3. Valid ScaleIEC Values

\begin{tabular}{ll}
\hline Input Value & Description \\
\hline 0 & No scaling: time series will remain as generated. \\
& $\begin{array}{l}\text { Scaling by HH value: all time series will be modified, using the same scaling } \\
\text { factor for each point (each component has separate scale). The hub point will } \\
\text { have the exact specified Tl; other points will not. }\end{array}$ \\
& $\begin{array}{l}\text { Independent scaling: all time series will be modified independently; scaling } \\
\text { factors vary by point and component. Each point will have the exact specified TI. }\end{array}$ \\
\hline
\end{tabular}

\section{NumGrid_Y: Number of Horizontal Grid Points [-]}

This parameter indicates the number of grid points in the horizontal direction, and it must be an integer greater than 1. If NumGrid_Y is an odd number, points fall along the undeflected tower centerline.

\section{TimeStep: Time Step [s]}

The TimeStep parameter is the time step in seconds (i.e., $\Delta t$ ). It is set to 0.05 seconds in the sample input files, and that value is recommended for most simulations. The time step determines the maximum frequency, $f_{\max }$, used in the inverse FFT:

$$
f_{\max }=1 / \Delta t
$$

\section{AnalysisTime: Length of Analysis [s]}

The AnalysisTime parameter is the length in seconds of the data to be analyzed (i.e., $\left.t_{\max }\right)$. This number dictates the frequencies which are used to generate the output time series. The following equations relate AnalysisTime to the frequency, $f$, and the number of frequencies, NumFreq:

$$
\begin{gathered}
\Delta f=\frac{1}{\text { AnalysisTime }} \\
\text { NumFreq }=\frac{\text { AnalysisTime }}{\text { TimeStep }}
\end{gathered}
$$

It is recommended that AnalysisTime be at least 600 seconds. To speed up the inverse FFT computations, TurbSim might add a few extra time steps to ensure that the number of analysis time steps is a product of small prime numbers. Extra time steps also are added if the length of the output time series is less than the AnalysisTime (see the discussion of the UsableTime parameter below).

\section{UsableTime: Usable Time Series Length [s]}

This parameter is the usable length (in seconds) of the data to output. This number differs slightly from the actual amount of data that TurbSim outputs. Because AeroDyn requires that there be data both upwind and downwind of the tower in case the turbine is yawed, it mandates that there be extra data in the FF files to shift the data enough to ensure that the turbine resides 
entirely within the wind-data domain. TurbSim always adds the amount of time equal to the grid width divided by the mean $\mathrm{HH}$ wind speed, $\bar{u}_{h u b}$, to the requested amount of usable time:

$$
\text { OutputTime }=\text { UsableTime }+\frac{\text { GridWidth }}{\bar{u}_{h u b}} .
$$

The analysis time must be at least as large as the output time:

$$
\text { AnalysisTime } \geq \text { OutputTime } \text {. }
$$

If necessary, TurbSim increases AnalysisTime to satisfy this relationship.

\section{HubHt: Turbine Hub Height [m]}

The HubHt parameter is hub height of the turbine for which the inflow is being generated. TurbSim uses the metric system so enter the value in meters. This parameter is used as a reference height for determining the grid location.

\section{GridHeight: Height of the Grid [m]}

This parameter is the distance (in meters) between the top and bottom of the grid. The top of the grid is assumed to be aligned with the top of the rotor disk (see Figure 5), and because all points of the grid must be above ground level, $\frac{1}{2}$ GridHeight $<H u b H t$.

When choosing a value for GridHeight, keep in mind that AeroDyn does not allow any part of the blade - including all system displacements - to lie outside the FF grid. The grid height must be large enough to encompass the entire rotor disk of FF files. See the parameter GridWidth for further discussion.

\section{GridWidth: Width of the Grid [m]}

This parameter is the width of the grid in meters. The rotor is assumed to be centered horizontally on the grid. If you are generating FF files for AeroDyn, the grid width-like the height — must be large enough to ensure that no part of the blade lies outside the grid, even when the system is displaced.

TurbSim assumes that the diameter of the rotor disk is the smaller of the GridHeight and GridWidth values. Because AeroDyn must interpolate within the grid for any point at which it

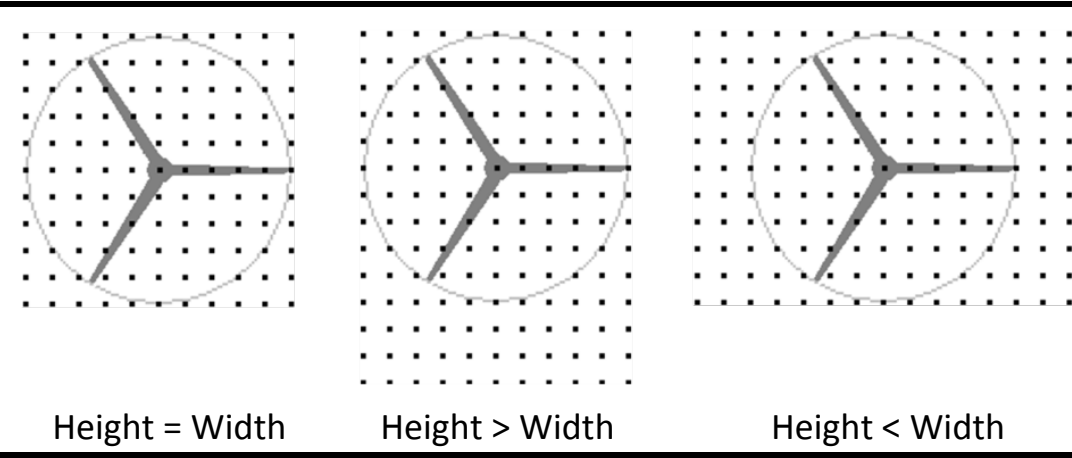

Figure 5. Example grid and rotor placements: the circles pictured here are the rotor diameters assumed by TurbSim; the actual rotor diameter(s) will be smaller than pictured 
needs wind speeds (i.e., AeroDyn cannot extrapolate), GridHeight and GridWidth should be larger than the rotor diameter. In fact, AeroDyn warns users if the grid width and height are not at least 10\% larger than the rotor diameter. For turbines that move a lot during simulation (e.g., floating wind turbines), the grid might have to be even larger.

As pictured in Figure 5, the hub is in the horizontal center of the grid, and the turbine hub height plus assumed rotor radius determines the top of the grid.

\section{VFlowAng: Mean Vertical Flow Angle [ $\left.{ }^{\circ}\right]$}

This parameter is the mean vertical angle of the wind, which is constant across the entire grid. Enter the angle in degrees, and do not exceed $45^{\circ}$ in magnitude. A positive value means that the wind is blowing uphill; a negative value indicates that the wind is blowing downhill. See HFlowAng and Figure 6 for more details.

\section{HFlowAng: Mean Horizontal Flow Angle $\left[{ }^{\circ}\right]$}

This parameter is the mean horizontal (crosswise) angle of the wind in degrees. In all cases except the GP_LLJ model, the horizontal flow angle is constant across the entire grid. For the GP LLJ model, which introduces direction shear with height, HFlowAng is the horizontal angle at hub height.

The mean flow angles VFlowAng and HFlowAng are used to rotate the wind from its alignment with the mean flow to the inertial reference frame. Users should be cautious, however, because AeroDyn - in its implementation of Taylor's frozen turbulence hypothesis - marches FF grids through the turbine along the positive $X$ axis at the mean hub-height wind speed, without regard to the flow angles (see Figure 6). This could give strange results if the mean flow angles are not small (for example, if HFlowAng $=180^{\circ}$, the grids move through the turbine in the opposite direction the wind is blowing). We recommend using a yaw error in the turbine simulation
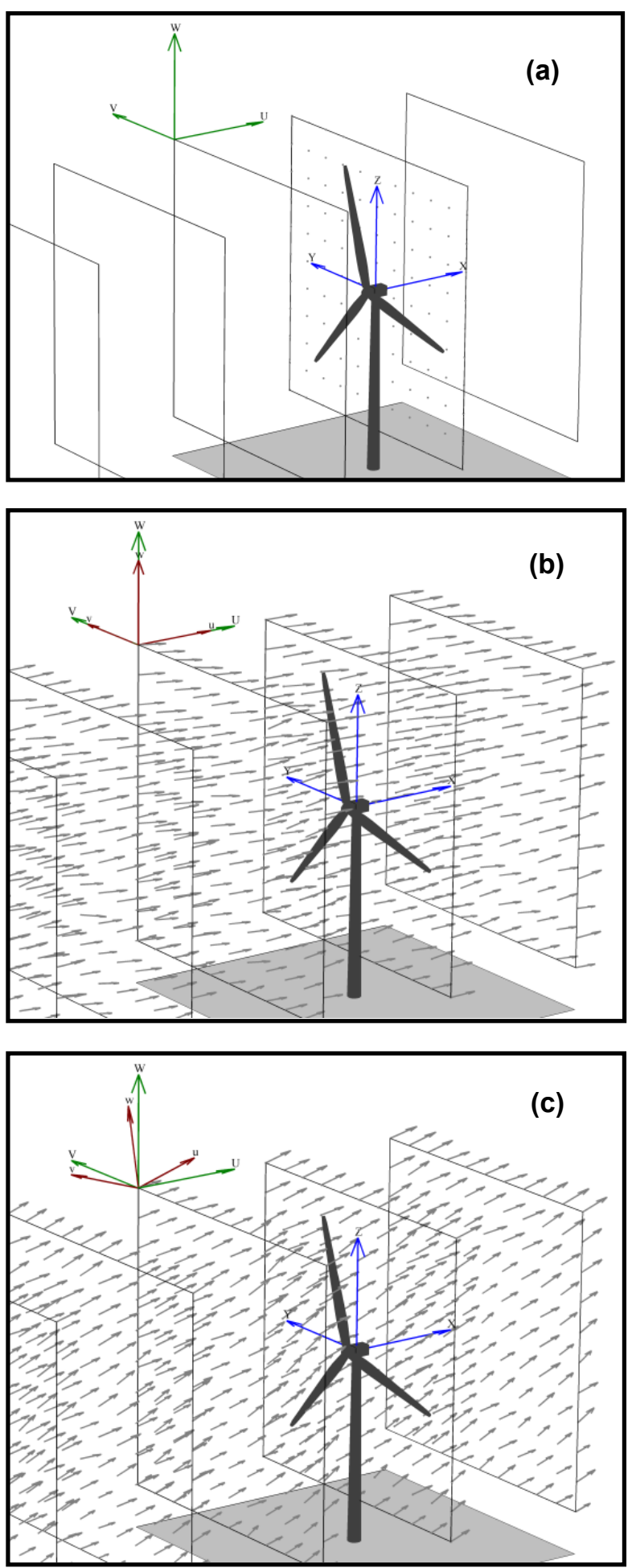

Figure 6. Example of TurbSim grids as implemented in AeroDyn: (a) The inertial frame coordinate systems and planes "marching" along positive $X$, regardless of flow angles, (b) wind field with both flow angles $0^{\circ}$, (c) the same wind field with VFlowAng $=8^{\circ}$ and HFlowAng $=15^{\circ}$ 
rather than using the HFlowAng parameter and using only small angles (e.g., less than $10^{\circ}$ ) for VFlowAng.

\section{Meteorological Boundary Conditions}

The Meteorological Boundary Conditions section of the TurbSim input file sets the spectral model to simulate, determines the mean wind speeds, and sets the boundary conditions for the spectral models defined in the IEC standards. Appendix C contains flow charts showing the function of the input parameters from this section.

\section{TurbModel: Turbulence Model [-]}

The TurbModel parameter tells TurbSim which spectral model it should use. Enter the six-character input value of the desired spectral model. Valid values are found in Table 4. For more information on these models, see the Spectral Models section in this document.

\section{IECstandard: IEC Standard [-]}

This input parameter tells TurbSim which IEC standard to use. Enter "1" to use the scaling from the IEC 61400-1 [21] standard or enter " 2 " or " 3 " to use the scaling from the IEC 61400-2 (small wind turbine) [22] or -3 (offshore wind turbine) [23] standards. To use the scaling parameters from the second edition of the IEC 61400-1 standard [24], follow the input with the string “-ED2" (i.e., "1-ED2"). Likewise, to use the scaling parameters from IEC 61400-1, 3 rd ed. [21], input the string "1-ED3". If the 61400-1 edition number is not specified, TurbSim uses the scaling from the third edition of IEC 61400-1 for the Kaimal model and scaling from the standard's second edition for the von Karman model (which is not defined in the newer edition). This input parameter is used only if the spectral model is IECKAI or IECVKM.

\section{IECturbc: IEC Turbulence [\%]}

The IECturbc parameter tells TurbSim what turbulence intensity you want to use with the IEC Kaimal or von Karman spectral models. Input values of " $A$," "B," or " $C$ " correspond to the standard IEC categories of turbulence characteristics, with " $\mathrm{A}$ " being the most turbulent. Figure 7 contains the relationship between wind speed and standard deviation for the standard IEC categories and turbulence types. You can also specify the TI in percent instead of choosing the turbulence categories. In this case, the standard deviation of the longitudinal wind speed, $\sigma_{1}$, is

Table 4. Valid TurbSim Spectral Models

\begin{tabular}{ll}
\hline 6-Character Input Value & Description \\
\hline GP_LLJ & NREL Great Plains low-level jet \\
IECKAI & IEC Kaimal \\
IECVKM & IEC von Karman \\
\hline NWTCUP & NREL National Wind Technology Center \\
SMOOTH & Risø smooth terrain \\
WF_07D & NREL wind farm: 7 rotor-diameters downwind \\
\hline WF_14D & NREL wind farm: 14 rotor-diameters downwind \\
WF_UPW & NREL wind farm: upwind \\
\hline
\end{tabular}


calculated using the following equation:

$$
\sigma_{1}=\frac{I E C t u r b c}{100} \bar{u}_{h u b} .
$$

If you use the NWTCUP spectral model and enter the string "KHTEST" for the IECturbc parameter, TurbSim creates a test wind field that can be used to see the effects of a KH billow. With this test function, TurbSim overrides the inputs for Richardson number (0.02); power-law coefficient (0.3); and billow type, size, and location. An LES-type billow centered on the rotor disk is scaled so that the billow achieves a bandwidth of at least $25 \mathrm{~Hz}$ and so that the expected maximum coherent turbulent kinetic energy (CTKE), defined as

$$
C T K E=\frac{1}{2} \sqrt{\left(u^{\prime} w^{\prime}\right)^{2}+\left(u^{\prime} v^{\prime}\right)^{2}+\left(v^{\prime} w^{\prime}\right)^{2}}
$$

is at least $30 \mathrm{~m}^{2} / \mathrm{s}^{2}$. This billow lasts at least half of the usable length of the output time series, and starts a quarter of the way through the time series. An example of KHTEST is presented in Figure 8.

The IECturbc parameter is not used for any other spectral model.

\section{IEC_WindType: IEC Turbulence Model [-]}

This parameter indicates which IEC wind model will be used. Valid entries, which are found in Table 5, include the Normal Turbulence Model (NTM), Extreme Turbulence Model (ETM), and Extreme Wind Speed Model (EWM) using the 10-minute average wind speed with a recurrence period of 1 year or 50 years. Note that the EWM scaling parameters in TurbSim are valid only for 10-minute simulations. The definitions of these models and of the wind turbine classes can be found in the IEC 61400-1 standard ( $3^{\text {rd }}$ ed.) [21]. If the IECturbc parameter was specified as a percentage instead of as a standard turbulence category, the wind model must be "NTM." This input is used only with the IEC spectral models.

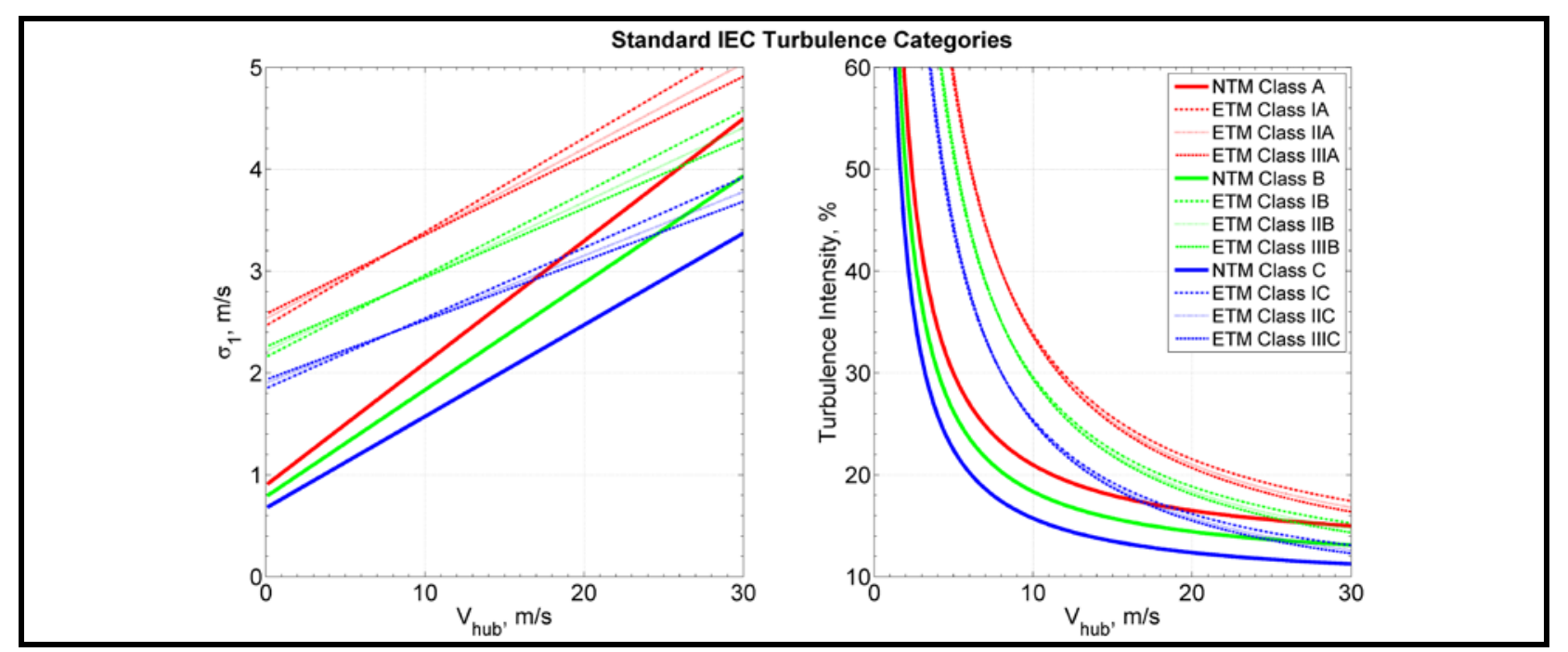

Figure 7. Longitudinal wind-speed standard deviation and TI for IEC turbulence categories as functions of the mean hub-height wind speed, $V_{\text {hub }}$ 


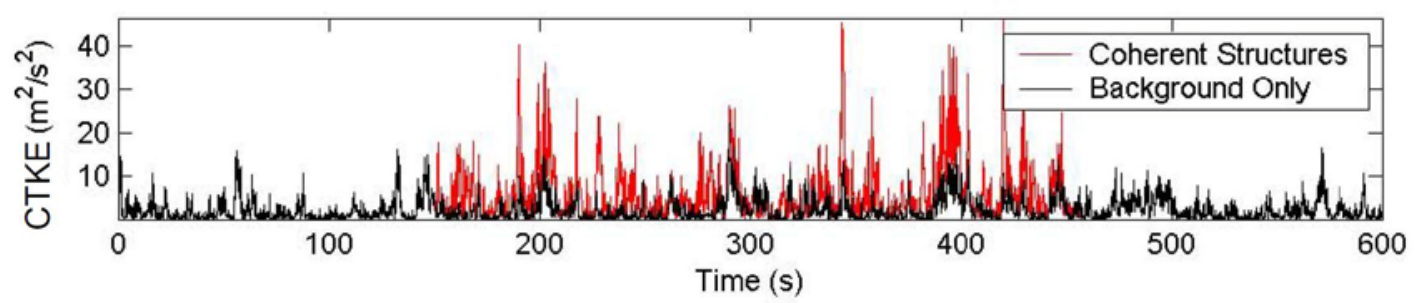

Figure 8. Coherent turbulent kinetic energy (CTKE) of an example simulation using KHTEST: the coherent structure placement in the middle of the time series is shown by the red lines

\section{ETMc: Extreme Turbulence Model Parameter c [m/s]}

The ETMc input parameter is the value of the variable $c$ in the equation for the longitudinal component standard deviation, $\sigma_{1}$, in the ETM (see Eq. 19 in section 6.3.2.3 of IEC 61400-1 $3^{\text {rd }}$ ed. [21]):

$$
\sigma_{1}=c I_{\text {ref }}\left(0.072\left(\frac{V_{\text {ave }}}{c}+3\right)\left(\frac{\bar{u}_{h u b}}{c}-4\right)+10\right) \text {. }
$$

The values for the variables $I_{\text {ref }}$ and $V_{\text {ave }}$-defined respectively as the expected value of turbulence intensity and $20 \%$ of the reference wind-speed average - are determined by the wind turbine class. Enter a value for $c$ in meters per second, or enter "default" for TurbSim to use $c=2 \mathrm{~m} / \mathrm{s}$, as defined in the standard. This parameter is used only with the Extreme Turbulence Model (i.e., when IEC_WindType $=x \mathrm{ETM}$ ).

\section{WindProfileType: Type of Wind Profile [-]}

The WindProfileType parameter tells TurbSim how to calculate the mean wind profile. Valid entries are found in Table 6. Users can enter the string "default" here for TurbSim to pick a wind profile based on the spectral model. The GP_LLJ model defaults to the JET profile and all others

Table 5. Valid IEC Turbulence Models

\begin{tabular}{ll}
\hline IEC_WindType & Description \\
\hline NTM & Normal Turbulence Model \\
1ETM & Class I Extreme Turbulence Model \\
2ETM & Class II Extreme Turbulence Model \\
3ETM & Class III Extreme Turbulence Model \\
\hline 1EWM1 & Class I turbulent Extreme Wind Speed Model, 1-yr recurrence \\
2EWM1 & Class II turbulent Extreme Wind Speed Model, 1-yr recurrence \\
3EWM1 & Class III turbulent Extreme Wind Speed Model, 1-yr recurrence \\
\hline 1EWM50 & Class I turbulent Extreme Wind Speed Model, 50-yr recurrence \\
2EWM50 & Class II turbulent Extreme Wind Speed Model, 50-yr recurrence \\
3EWM50 & Class III turbulent Extreme Wind Speed Model, 50-yr recurrence \\
\hline
\end{tabular}


default to the IEC profile. Please see the Wind Profiles section of this guide for more details about the different wind profile types.

\section{RefHt: Reference Height [m]}

The RefHt parameter specifies the height (in meters) of the corresponding reference wind speed (parameter URef). This parameter enables users to specify the mean wind speed at a height other than the hub height. TurbSim uses this reference height and wind speed with the wind profile type to calculate the HH mean wind speed. The reference height also is used with URef and the surface roughness (parameter Z0) to compute default input values for parameters UStar and ZI.

\section{URef: Reference Wind Speed [m/s]}

The URef parameter is the mean streamwise wind speed at the reference height. It is the mean value over the entire AnalysisTime length of the simulation of the $u$-component wind speed. It must be a positive value in units of meters per second. If you use "JET" for the WindProfileType parameter, you can enter the string "default" here for TurbSim to calculate a default wind speed in two steps: (1) TurbSim calculates the maximum speed of the jet wind profile, $\bar{u}_{\text {JetMax }}$, based on the jet height, ZJetMax, and a random variate (shown in Figure 9) then (2) it calculates the wind speed at RefHt using $\bar{u}_{\text {JetMax }}$ along with parameters ZJetMax, RICH_NO, and UStar. The calculations of the low-level jet wind speed profile are discussed further in the Wind Profiles section of this guide.

\section{ZJetMax: Height of the Jet [m]}

The ZJetMax parameter is the height in meters of the low-level jet. Enter the approximate height at which the low-level jet wind profile reaches its maximum wind speed, or enter the string "default" to have TurbSim calculate a jet height. The default height is a function of parameters RICH_NO and Ustar with a random component based on LLLJP measurements. The default height - without the random component—is plotted in Figure 10. ZJetMax, which must be a value between $70 \mathrm{~m}$ and $490 \mathrm{~m}$, is used to calculate the mean wind speed and direction profiles. It is used only when WindProfileType is "JET."

\section{PLExp: Power-Law Exponent [-]}

The PLExp parameter is used to compute the mean $u$-component wind speeds across the rotor disk when WindProfileType is "IEC" or "PL." It is the exponent used to define the power-law wind profile,

Table 6. Valid Wind Profile Types

\begin{tabular}{ll}
\hline WindProfileType & Description \\
\hline PL & Power-law wind profile \\
LOG & Diabatic (logarithmic) wind profile; not valid with KHTEST \\
JET & Low-level jet wind profile, valid only with GP_LLJ model \\
IEC & Power-law profile on the rotor disk; logarithmic profile elsewhere \\
Default & Uses a default: JET for the GP_LLJ model; IEC for all other models \\
\hline
\end{tabular}




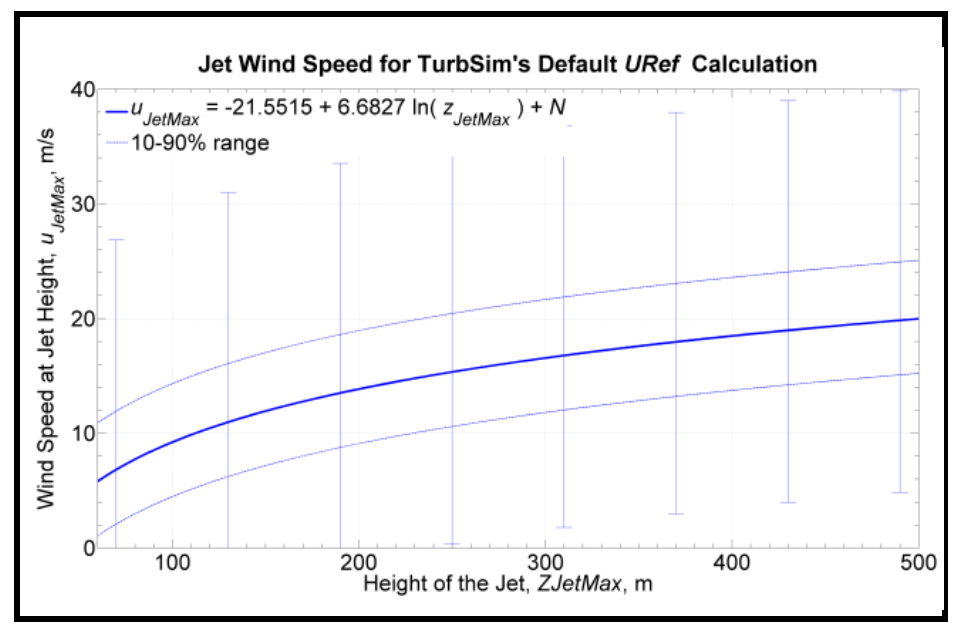

Figure 9. Default jet wind speed for URef calculation: error bars indicate the range of random variate, $N$; dotted lines mark the tenth and ninetieth percentiles

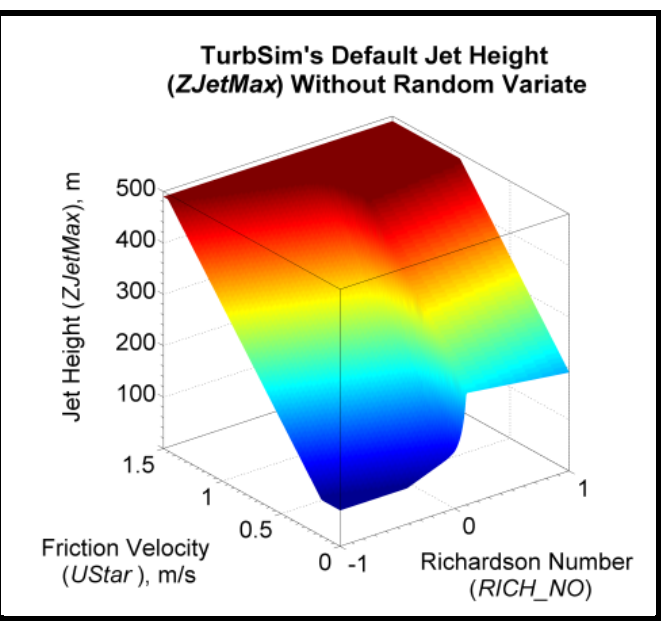

Figure 10. Default jet height, ZJetMax, without random variates (the random variation range is approximately $\pm 50 \mathrm{~m}$ )

$$
\bar{u}(z)=\bar{u}_{h u b}\left(\frac{z}{H u b H t}\right)^{\text {PLExp }}
$$

where $\mathrm{z}$ is the height above ground level. The exponent can be positive, negative, or zero (for no shear). Enter the string "default" to have TurbSim use a default value based on the specified spectral model, as shown in Table 7. If KHTEST is specified for parameter IECturbc, the PLExp parameter is overwritten to 0.3 .

\section{Z0: Surface Roughness Length [m]}

The surface roughness length, Z0, is the last parameter in this section. This length - a measure of the roughness of the surface terrain - is the extrapolated height at which the mean wind speed becomes zero in a neutral atmosphere, assuming a logarithmic vertical wind profile:

$$
\bar{u}(z)=U R e f \frac{\ln (z / z 0)}{\ln (\operatorname{RefH} t / z 0)} .
$$

Enter the length in meters, or enter the string "default" to have TurbSim use a default value based on the specified spectral model. The default values are listed in Table 7.

\section{Non-IEC Meteorological Boundary Conditions}

If you have specified either the Kaimal or von Karman spectral model, TurbSim does not read this section of the input file. The other (non-IEC) spectral models require the additional meteorological boundary conditions contained in this section. All of the inputs in this section, with the exception of the gradient Richardson number, can be replaced with the string "default." Appendix $\mathrm{C}$ contains flow charts showing the function of the input parameters from this section and how the default values are chosen. 
Table 7. Default Inputs for Meteorological Boundary Conditions

\begin{tabular}{lll}
\hline TurbModel & PLExp & Z0 (m) \\
\cline { 2 - 3 } & $\begin{array}{l}0.11 \text { for EWM } \\
\text { IECKAI, IECVKM }\end{array}$ & $\begin{array}{l}0.14 \text { for offshore }(61400-3) \text { NTM, } \\
0.2 \text { otherwise }\end{array}$ \\
SMOOTH & 0.143 & 0.03 \\
GP_LLJ & 0.143 & 0.01 \\
NWTCUP & $0.08-0.15$, increasing with RICH_NO, & 0.005 \\
WF_UPW & 0.3 for KHTEST option & 0.021 \\
WF_07D & same as NWTCUP & 0.018 \\
WF_14D & $0.13-0.18$, increasing with RICH_NO & 0.064 \\
\hline
\end{tabular}

\section{Latitude: Site Latitude [ $\left.{ }^{\circ}\right]$}

The first parameter in this section is the site latitude in degrees. The latitude is used only to calculate a Coriolis term in the default mixing layer depth (parameter $Z I$ ). The magnitude of the latitude must be between $5^{\circ}$ and $90^{\circ}$; the default value is $45^{\circ}$.

\section{RICH_NO: Gradient Richardson Number [-]}

The $R \bar{I} C H \_N O$ parameter is the turbine-layer vertical stability given by the dimensionless gradient Richardson number, which is defined as

$$
R I C H_{-} N O=\frac{\frac{g}{\bar{\theta}} \frac{\partial \bar{\theta}}{\partial z}}{\left(\frac{\partial \bar{u}}{\partial z}\right)^{2}} .
$$

In this equation, $g$ is the gravitational acceleration, $z$ is the height above ground, and $u$ is the wind speed. The variable $\theta$ represents potential temperature, which is calculated using the mean absolute air temperature, $T$, and atmospheric pressure, $p$ :

$$
\theta=T\left(\frac{1000}{p}\right)^{0.286}
$$

The RICH_NO parameter is used to calculate the velocity spectra and the JET and LOG wind profiles, scale coherent structures, and determine default values for many input parameters.

Enter zero for neutral conditions, a negative value for unstable conditions, or a positive number for stable atmospheric conditions. The GP_LLJ and NWTCUP models limit this input to $-1 \leq \mathrm{RICH}_{-} N O \leq 1$. If KHTEST is specified for parameter IECturbc, the RICH_NO parameter is overwritten to 0.02 . The $R I C H \_N O$ parameter does not accept the value "default." 
UStar: Rotor-Disk Average Friction Velocity [m/s]

The parameter UStar is the friction or shear velocity, $u_{*}$, averaged over the rotor disk:

$$
\begin{aligned}
\text { UStar } & =\overline{u_{*}} \\
& =\frac{1}{n_{p}} \sum_{i=1}^{n_{p}} \sqrt{\left|\overline{u^{\prime} w^{\prime}}\right|_{i}},
\end{aligned}
$$

where the prime quantities indicate the fluctuating (zero-mean) longitudinal $(u)$ and vertical $(w)$ wind components at $n_{p}$ measurement points on the rotor disk. The GP_LLJ model, which scales the velocity spectra with local friction velocities ( $u_{*}$ values varying with height), assumes that UStar is the average friction velocity of three points on the $u_{*}$ profile: one at the hub, one at the top of the rotor, and one at the bottom of the rotor.

UStar is used to scale the velocity spectra of non-IEC spectral models, to scale the JET windspeed and wind-direction profiles, and to calculate the default values of many input parameters. Enter UStar in units of meters per second or enter "default" to have TurbSim calculate an appropriate value. The default value is calculated using the diabatic $u_{* 0}$ (near the surface), which is predicted by Panofsky and Dutton's modified logarithmic profile [25] using

$$
u_{*_{0}}=\frac{0.4 U R e f}{\ln \left(\operatorname{RefHt} / \mathrm{ZO}_{0}\right)-\Psi_{M}\left(\mathrm{RICH}_{-} N O\right)},
$$

where $\Psi_{M}$ is a function that depends on the $\mathrm{RICH}_{-} N O$ stability parameter. The relationship between RICH_NO and $u_{* 0}$, normalized by URef, at RefHt $=80 \mathrm{~m}$ is plotted in Figure 11 . The relationship between $u_{*_{0}}$ and the default UStar is shown in Figure 12.

If "default" is entered for the reference wind speed, URef, the string "default" cannot be entered for the UStar parameter, because the default values for the two parameters are interdependent.

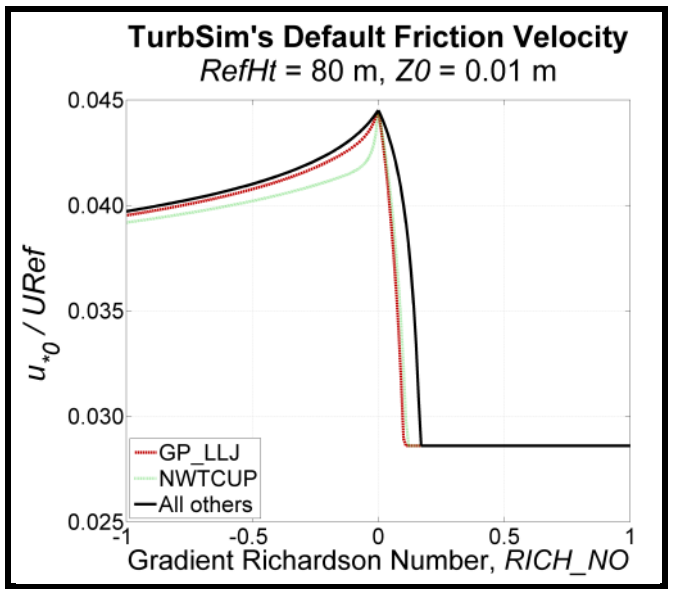

Figure 11. Diabatic friction velocity, $u_{*_{0}}$, normalized by URef and calculated using RefHt $=80 \mathrm{~m}$ and $\mathrm{ZO}=0.01 \mathrm{~m}$

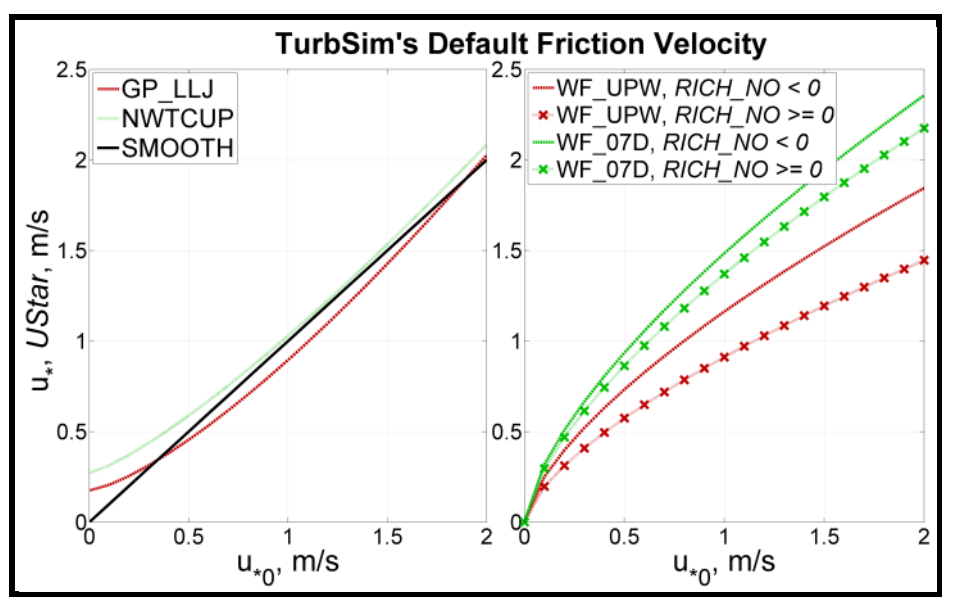

Figure 12. Default UStar as a function of diabatic friction velocity, $u_{*_{0}}$ (left: SMOOTH, GP_LLJ, and NWTCUP models, right: wind farm models) 


\section{ZI: Depth of the Mixing Layer [m]}

The parameter $Z I$ is the depth of the mixing layer (in meters). This parameter scales the velocity spectra in unstable atmospheric conditions and is not used for stable atmospheric conditions. The default mixing layer depth is calculated using

$$
Z I= \begin{cases}\frac{400 \text { URef }}{\log _{10}\left(\frac{\text { RefHt }}{Z 0}\right)}, & \text { UStar }<u_{*_{0}} \\ \frac{U \text { Star }}{12.0 \omega \sin \left(\frac{\pi}{180} \mid \text { Latitude } \mid\right)}, & \text { UStar } \geq u_{*_{0}},\end{cases}
$$

where $\omega=7.292116 \times 10^{-5} \mathrm{rad} / \mathrm{s}$ is the Earth's angular speed of rotation. This equation combines the work of Dutton et al. [26] with the ESDU [27].

\section{PC_UW: Average u'w' Reynolds Stress at the $\mathrm{Hub}\left[\mathrm{m}^{2} / \mathrm{s}^{2}\right]$}

The $P C$ C $U W$ parameter is the desired average $u^{\prime} w^{\prime}$ Reynolds stress $\left(\mathrm{in} \mathrm{m}^{2} / \mathrm{s}^{2}\right)$ at the simulated hub point. It is used in conjunction with the next two inputs, parameters $P C_{-} U V$ and $P C_{-} V W$, to create some correlation between the wind-speed components.

TurbSim modifies the $v$ - and $w$-component wind speeds (for non-IEC models only) by computing a linear combination of the time series of the three independent wind-speed components to obtain the mean Reynolds stresses $P_{C} U W, P C_{-} U V$, and $P C_{-} V W$ at the hub point. The linear combinations are computed for each point, $j$, using the equations

$$
\begin{aligned}
u_{j, \text { correlated }}^{\prime} & =u_{j, \text { independent }}^{\prime} \\
v_{j, \text { correlated }}^{\prime} & =\alpha_{u v} u_{j, \text { independent }}^{\prime}+v_{j, \text { independent }}^{\prime}+\alpha_{v w} w_{j, \text { independent }}^{\prime} \\
w_{j, \text { correlated }}^{\prime} & =\alpha_{u w} u_{j, \text { independent }}^{\prime}+w_{j, \text { independent }}^{\prime}
\end{aligned}
$$

The three $\alpha$ variables are coefficients chosen to generate the desired Reynolds stresses for the correlated wind components at the hub:

$$
\begin{gathered}
P C_{-} U W=\overline{u_{\text {hub,correlated }}^{\prime} w_{\text {hub,correlated }}^{\prime}} \\
P C_{-} U V=\overline{u_{\text {hub,correlated }}^{\prime} v_{\text {hub,correlated }}^{\prime}} \\
P C_{-} V W=\overline{v_{\text {hub,correlated }}^{\prime} w_{\text {hub,correlated }}^{\prime}} .
\end{gathered}
$$

Because this method affects the frequency domain somewhat, we have placed the following limit on the coefficients: $|\alpha| \leq 1$. This limit can cause the actual hub Reynolds stresses to differ from the desired values.

Enter the string "default" for TurbSim to compute an appropriate Reynolds stress for PC_UW. The default value for the SMOOTH model is the same as that for the WF_UPW and WF_07D models: PC_UW $=-U S t a r^{2}$. The default value for the WF_14D model has the same magnitude, but is positive $1 \%$ of the time (randomly). The magnitudes of the defaults for the NWTCUP and GP_LLJ models are functions of UStar, RICH_NO, height, mean hub-height wind speed, and shear across the rotor disk. The signs of the defaults are determined randomly, 
with the probability that $P C_{-} U W$ is negative increasing with the magnitude of the default. Users can also enter the string "none" to set $\alpha_{u w}=0$ and disable the correlation between the $u$ and $w$ components.

\section{PC_UV: Average u'v' Reynolds Stress at the Hub $\left[\mathrm{m}^{2} / \mathrm{s}^{2}\right]$}

The $P C \_U V$ parameter is the desired average $u^{\prime} v^{\prime}$ Reynolds stress (in $\mathrm{m}^{2} / \mathrm{s}^{2}$ ) at the simulated hub point. It is used in conjunction with the parameters $P C_{-} U W$ and $P C_{-} V W$ to create cross-

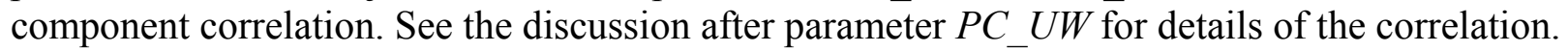

To set $\alpha_{u v}=0$ and disable the correlation between the $u$ and $v$ components, enter the string "none." Users also can enter the string "default" if you would like TurbSim to compute a default value for $P C_{-} U V$. The magnitudes of the defaults for site-specific models (GP_LLJ, NWTCUP, WF_UPW, WF_07D, and WF_14D) are functions of UStar, RICH_NO, height, mean hub-height wind speed, and shear across the rotor disk. The signs of the defaults are determined randomly. The default for the SMOOTH model is "none."

\section{PC_VW: Average v'w' Reynolds Stress at the Hub $\left[\mathrm{m}^{2} / \mathrm{s}^{2}\right]$}

The $P C_{-} V W$ parameter is the desired average $v^{\prime} w^{\prime}$ Reynolds stress (in $\mathrm{m}^{2} / \mathrm{s}^{2}$ ) at the simulated hub point. It is used in conjunction with the parameters $P C_{-} U W$ and $P C_{-} U V$ to create crosscomponent correlation. See the discussion after parameter $P C_{-} U W \overline{\text { for details. }}$

Users can enter the string "none" to set $\alpha_{v w}=0$ and disable the correlation between the $v$ and $w$ components. To have TurbSim compute a default value for $P C_{-} V W$, enter the string "default." The magnitudes of the defaults for site-specific models are functions of UStar, RICH_NO, height, mean hub-height wind speed, and shear across the rotor disk. The signs of the defaults are determined randomly. The default for the SMOOTH model is "none."

\section{IncDec1: Spatial Coherence for the u-Component Wind Speed [-, $\left.\mathrm{m}^{-1}\right]$}

The IncDecl parameter defines the spatial coherence decrement, $a$, and offset parameter, $b$, for the $u$-component wind speed $(K=u)$. These two values are used to define the degree of spatial coherence between points on the grid using the definition

$$
\operatorname{Coh}_{i, j}=\exp \left(-a_{K}\left(\frac{r}{z_{m}}\right)^{\operatorname{CohExp}} \sqrt{\left(\frac{f r}{\bar{u}_{m}}\right)^{2}+\left(b_{K} r\right)^{2}}\right),
$$

where $r$ is the distance between points $i$ and $j, f$ is the cyclic frequency, CohExp is the coherence exponent input parameter, and $z_{m}$ and $u_{m}$ are the mean height and wind speed of points $i$ and $j$. Please see the Spatial Coherence Models section of this document for more information.

The IncDecl decrement, $a$, must be a positive number. Users can enter "default" or both the $a$ and $b$ coherence parameters in quotation marks on the same line. For example, "10.0 0.1E-02" uses a coherence decrement of $a=10.0$ and an offset parameter of $b=0.1 \mathrm{E}-02 \mathrm{~m}^{-1}$. Omitting the quotation marks around the two input parameters causes TurbSim to use $b=0$.

The default $a$ parameter for the $u$-component is $a_{u}=\bar{u}_{h u b}$ for the SMOOTH model. The other non-IEC models base this default value on measured vertical coherence spectra from their 
respective datasets. The default $a$ parameter for these models is a function of $\mathrm{HubHt}$ and RICH_NO parameters, as well as the mean hub-height wind speed.

The default $b$ parameter for the $u$-component is $b_{u}=0$ for the SMOOTH model. The other nonIEC models calculate the default $b$ parameter as a function of mean hub-height wind speed. The GP_LLJ and NWTCUP models also use the RICH_NO parameter to calculate the default $b$.

Figure 13 shows the default parameters for neutral conditions (i.e., $R I C H \_N O=0$ ) using a value of $80 \mathrm{~m}$ for the HubHt parameter.

\section{IncDec2: Spatial Coherence for the v-Component Wind Speed [-, $\left.\mathrm{m}^{-1}\right]$}

The IncDec2 parameter defines the spatial coherence decrement, $a$, and offset parameter, $b$, for the $v$-component wind speed using the coherence definition of Eq. (18) (with $K=v$ ). Users can enter "default" for TurbSim to pick appropriate values for both $a$ and $b$, or enter both $a$ and $b$ parameters in one set of quotation marks on the same line. See the discussion for IncDecl (above) for further details.

The default $a$ parameter for the $v$-component is $a_{v}=0.75 \bar{u}_{h u b}$ for the SMOOTH model. The other non-IEC models calculate the default value as a function of $\mathrm{HubHt}$ and $\mathrm{RICH}_{-} \mathrm{NO}$ parameters, as well as the mean hub-height wind speed.

The default $b$ parameter for the $v$-component is $b_{v}=0$ for the SMOOTH model. The other nonIEC models calculate the default $b$ parameter as a function of mean hub-height wind speed. The GP_LLJ and NWTCUP models also use the RICH_NO parameter to calculate the default $b$.

Figure 14 shows the default parameters for neutral conditions using an 80-m HubHt.

\section{IncDec3: Spatial Coherence for the w-Component Wind Speed [-, $\left.\mathrm{m}^{-1}\right]$}

The IncDec 3 parameter defines the spatial coherence decrement, $a$, and offset parameter, $b$, for the $w$-component wind speed using the coherence definition of Eq. (18) (with $K=w$ ). Users can enter "default" for TurbSim to pick appropriate values for both $a$ and $b$, or enter both $a$ and $b$

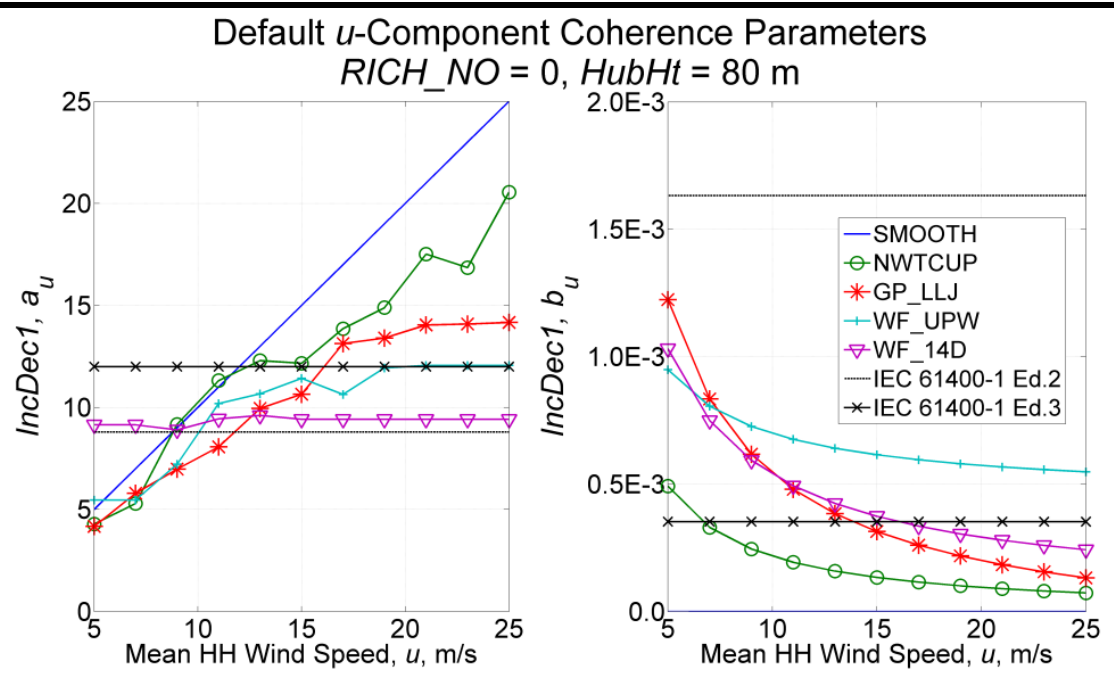

Figure 13. Default $u$-component coherence parameters, IncDec1, ( $a_{u}$ left, $b_{u}$ right) as a function of wind speed, using $\mathrm{RICH} \mathrm{NO}=0$ and $\mathrm{HubHt}=80 \mathrm{~m}$ (IEC values are plotted for comparison) 
parameters in one set of quotes on the same line. See the discussion for IncDecl for further details.

The default $a$ parameter for the $w$-component is $a_{w}=0.75 \bar{u}_{h u b}$ for the SMOOTH model and $a_{w}=0.4 a_{u}$ for the three wind farm models (WF_UPW, WF_07D, and WF_14D). The GP_LLJ and NWTCUP models calculate the default value as a function of the $\mathrm{HubHt}$ and RICH_NO parameters, as well as the mean hub-height wind speed.

The default $b$ parameter for the $w$-component is $b_{w}=0$ for the SMOOTH model and $b_{w}=10 b_{u}$ for the three wind farm models. The GP_LLJ and NWTCUP models calculate the default $b$ parameter as a function of mean hub-height wind speed and the $R I C H$ NO parameter. Figure 15 shows the default parameters for neutral conditions using an 80-m Hub $\overline{b t}$.

\section{CohExp: Coherence Exponent [-]}

The CohExp parameter is the exponent in the coherence definition of Eq. (18). The same value of CohExp is used for all three wind components; enter a non-negative number or "default" to use the default value of 0 .

\section{Coherent Turbulence Scaling Parameters}

The coherent turbulence scaling parameters found in this section are used with non-IEC spectral models when the gradient Richardson number $\left(\mathrm{RICH}_{-} \mathrm{NO}\right)$ is greater than -0.05 and the option to create coherent turbulence time-step files has been selected (i.e., $W r A C T=$ true). Appendix $\mathrm{C}$ contains a flow chart showing the functions of the input parameters from this section.

TurbSim uses empirical values to calculate when and how coherent events - pieces (sections in time) of a Kelvin-Helmholtz (KH) billow simulated using either direct numerical simulation (DNS) or large-eddy simulation (LES) — should be added to the background turbulence. It creates a coherent turbulence time-step file that AeroDyn can read. The super-positioning of

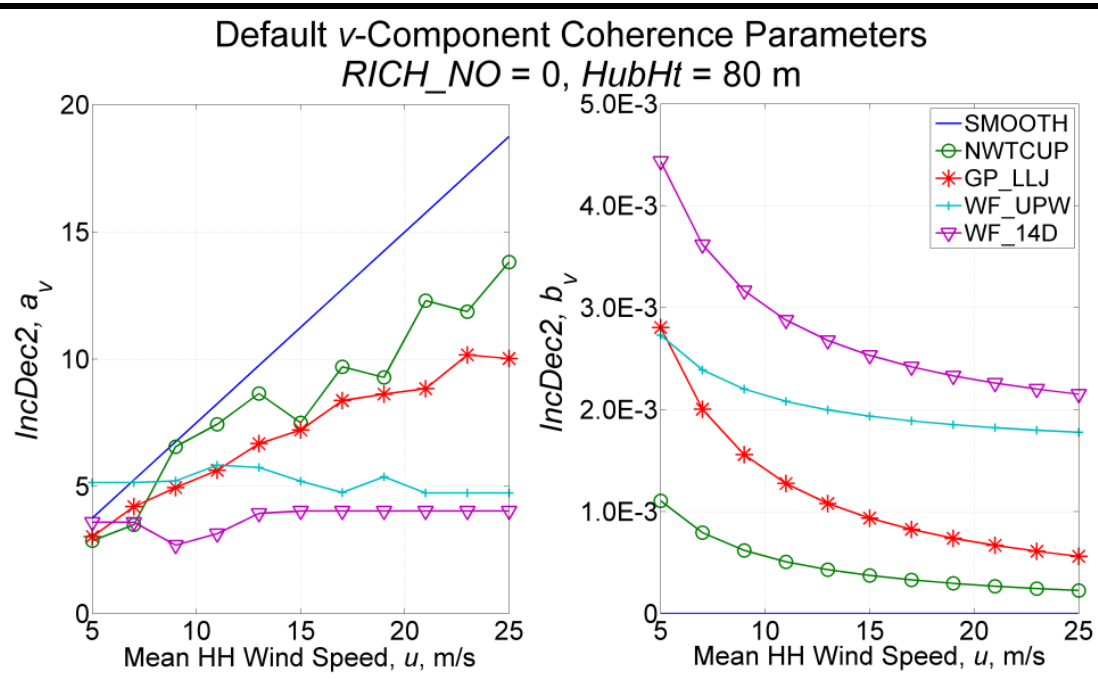

Figure 14. Default $v$-component coherence parameters, IncDec2, ( $a_{v}$ left, $b_{v}$ right) as a function of wind speed, using $\mathrm{RICH}$ NO $=0$ and $\mathrm{HubHt}=80 \mathrm{~m}$ (IEC does not define $v$ component coherence parameters) 


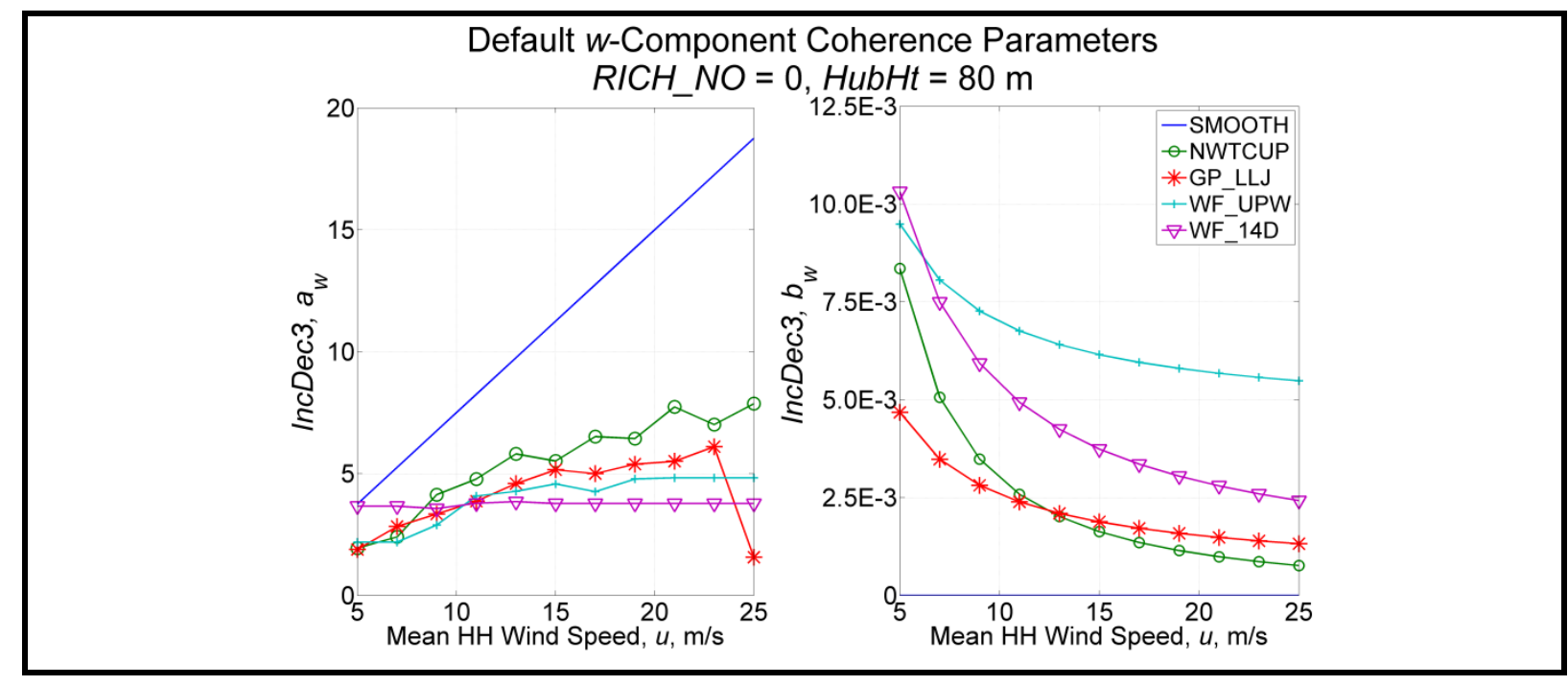

Figure 15. Default w-component coherence parameters, IncDec3, ( $a_{w}$ left, $b_{w}$ right) as a function of wind speed, using RICH_NO=0 and $\mathrm{HubHt}=80 \mathrm{~m}$ (IEC does not define $\mathbf{w}$ component coherence parameters)

coherent events on the background turbulence occurs in AeroDyn in the inertial reference frame coordinate system that AeroDyn uses. The Coherent Structures section of this document discusses this topic further.

\section{CTEventPath: Name of Coherent Turbulence Events path [-]}

The CTEventPath parameter is the name of the path that contains the coherent event definition files. Use quotation marks around the path name. This directory should contain files named "Events.les," "Events.dns," and "Events.xtm" as well as one or more files named "Event $x x x x x$.dat" (with digits replacing the $x x x x x$ ). These event definition files and the associated binary data files that AeroDyn reads are provided in the coherent structure archive on the TurbSim Web site (in folder "EventData").

\section{CTEventFile: Type of Coherent Events [-]}

This parameter tells TurbSim which type of coherent event files to use. Valid entries are found in Table 8. In each individual simulation, all events are of the same type (either all LES or all DNS). TurbSim automatically uses LES events when KHTEST is specified for parameter IECturbc.

\section{Randomize: Randomize Size and Location of KH Billow Pieces? [T/F]}

Set the Randomize parameter to "true" to randomize the size and location of the coherent structures in the rotor disk or to "false" to specify these values yourself. A value of "true" overrides the next three input parameters (DistScl, CTLy, and CTLz). Instead, the coherent structures are centered laterally and randomly chosen to cover either (1) the full rotor disk (75\% of the time), (2) only the lower half of the disk (12.5\% of the time), or (3) only the upper half $(12.5 \%$ of the time).

\section{DistScl: Disturbance Scale [-]}

The DistScl parameter is the disturbance scale, which determines the size of the coherent event data set relative to the rotor disk. It is the ratio of the height of the coherent dataset to the 
Table 8. Valid CTEventFile Entries

\begin{tabular}{ll}
\hline Input Value & Description \\
\hline DNS & Reads DNS event files (CTEventPath\Events.dns) \\
LES & Reads LES event files (CTEventPath $\backslash$ Events.les) \\
Random & Randomly chooses between LES and DNS (equal probability) \\
\hline
\end{tabular}

(assumed) rotor diameter. A value of 1.0 makes the coherent structures the height of the rotor disk; 0.5 makes them half the height of the rotor disk. If parameter IECturbc is KHTEST, TurbSim overrides DistScl with a value of 1.0. When Randomize is "true," the value of this input is overridden (as discussed above).

\section{CTLy: Lateral Location of Coherent Turbulence [-]}

This parameter laterally positions the coherent structures from the KH billow on the rotor disk. CTLy is the fractional location of the tower centerline from the right to left side (looking downwind) of the coherent event dataset. A value of 0.5 puts the tower centerline in the center of the billow. The coherent structures are periodic in the lateral direction so they cover the grid horizontally, regardless of the location of the tower centerline. Figure 16 shows coherent structure scaling with CTLy on the abscissa. If parameter IECturbc is KHTEST, TurbSim overrides CTLy with a value of 0.5 . When Randomize is "true," the value of this input is overridden (see the discussion of Randomize).

\section{CTLz: Vertical Location of Coherent Turbulence [-]}

This parameter positions the coherent structures vertically on the rotor disk. CTLz is the fractional location of hub height from the bottom of the dataset. A value of 0.5 places the vertical center of the billow at hub height. The structures are constant above and below the top and bottom of the dataset. Figure 16 shows how the structures are scaled. If parameter IECturbc is KHTEST, TurbSim overrides $C T L z$ with a value of 0.5 . When Randomize is "true," the value of this input also is overridden (see the discussion of Randomize).

\section{CTStartTime: Minimum Start Time for Coherent Turbulence [s]}

The CTStartTime parameter is used to determine where the first coherent structure will be placed in the time-step file. TurbSim ensures that the first event in the coherent time-step file does not occur before the time entered here (in seconds). This feature can be useful if you do not want a turbine to encounter coherent structures during the startup transient of a simulation.

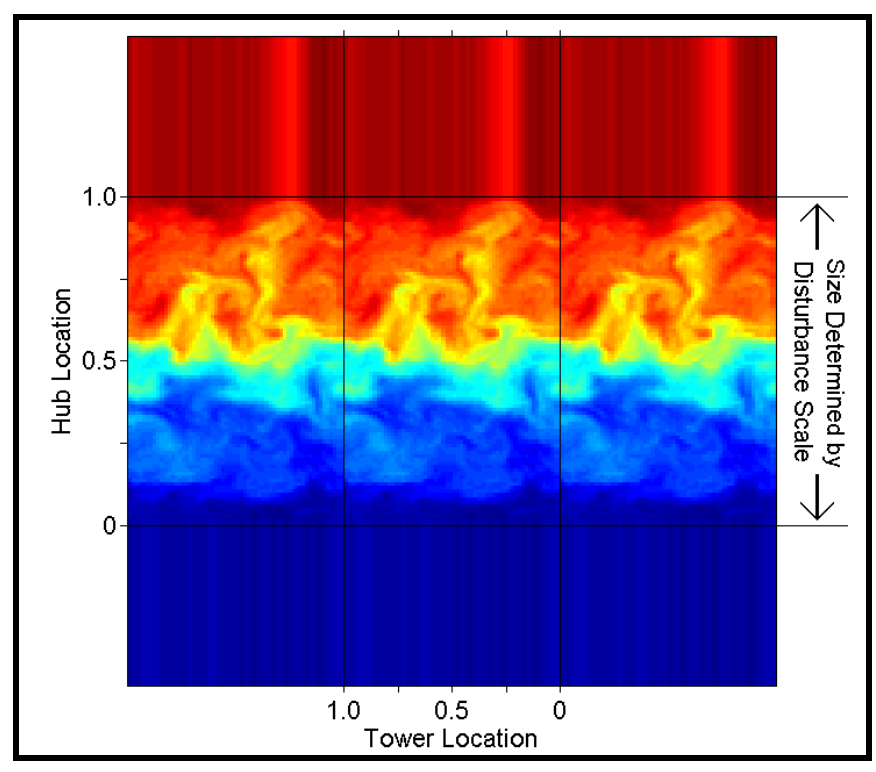

Figure 16. Coherent structure scaling (looking downwind): CTLy is the tower location, CTLz is the hub (height) location, and DistScl determines the size $(\mathrm{m})$ relative to the rotor disk 


\section{Output Files}

TurbSim can generate several different sets of output files. They have the root name of the TurbSim input file, and their extensions indicate what type of files they are. The Runtime Options section (above) describes how to tell TurbSim which sets to output.

\section{Summary Files}

TurbSim generates a summary file for all runs. This summary file is a text file with a ".sum" extension. The first part of the file tells you what was specified in the input file. After that, TurbSim prints out many statistics for the run. These statistics are calculated using the entire AnalysisTime so if a shorter UsableTime was requested, the statistics of the output time series could be different than what is displayed in the summary file. Also keep in mind that the turbulence statistics are for the background turbulence only; they do not include effects of any coherent structures generated in coherent turbulence time-step files. If a coherent turbulence time-step file is generated, TurbSim prints the number of events and the total length of those events in the summary file. If Bladed-style FF files or separate tower output are requested, TurbSim adds another section that tells AeroDyn how to convert the normalized data to floatingpoint form.

\section{Hub-Height Binary Files}

The hub-height binary files are in a machine-readable form designed to be read by GenPro, a postprocessor from the National Center for Atmospheric Research (NCAR). TurbSim gives these files a ".bin" extension. At each time step, TurbSim writes the values of a series of parameters in the binary file. The parameters are listed in Table 9 in the order in which they appear in the file. Each value is stored as a 4-byte floating-point (real) number. A MATLAB ${ }^{\circledR}$ script for reading these files is included in the TurbSim archive; it is named "TestlreadHHbin.m."

\section{Hub-Height Formatted Files}

The hub-height formatted files contain essentially the same information as the hub-height binary files, but the parameters are written in columns in human-readable form. See Table 9 for the list of parameters. These files have a ".dat" extension.

\section{Hub-Height AeroDyn Formatted Files}

These human-readable files are in a format compatible with AeroDyn. They have the ".hh" extension. See Table 10 for the file format; the AeroDyn User's Guide [1] contains a detailed description of the parameters. The horizontal wind speed and wind direction are equivalent to the vector sum of the instantaneous $U$ - and $V$-component time series from the hub-point, and the vertical wind speed is the corresponding $W$-component time series. TurbSim always sets the horizontal wind-shear, vertical linear wind-shear, and gust-speed parameters to zero in the AeroDyn hub-height files. The vertical power-law wind-shear exponent is constant for the entire time series. If the input wind-profile type (WindProfileType) is PL or IEC, the value in the AeroDyn HH file is the PLExp parameter; if WindProfileType is JET or LOG, the power law exponent is calculated based on the mean wind speeds at the top and bottom of the rotor disk:

$$
P L E x p=\ln \left(\frac{\bar{u}\left(z_{\text {top }}\right)}{\bar{u}\left(z_{\text {bottom }}\right)}\right) / \ln \left(\frac{z_{\text {top }}}{z_{\text {bottom }}}\right) .
$$


The column of plots on the right side of Figure 17 shows how AeroDyn uses the information in these $\mathrm{HH}$ files to produce wind speeds at any part of the volume surrounding the turbine.

\section{Full-Field TurbSim Binary Files}

The FF TurbSim binary files are designed to be read by AeroDyn. They have a ".bts" extension. (The column of plots on the left side of Figure 17 shows how AeroDyn uses FF data.) TurbSim normalizes the time-series data (in the inertial reference frame coordinate system) and encodes them in 2-byte integers stored in these files. The first part of each file is a header that provides information about the grid and tells AeroDyn how to convert the integers to floating-point values. The wind speeds for the NumGrid_Y $Y \times$ NumGrid_Z grids and the tower points (if specified) follow that. See Appendix D in this document for the file format. A MATLAB script for reading these files is included in the TurbSim archive; it is named "Test $\backslash$ readTSgrid.m."

This binary format has been designed so that AeroDyn does not need to read any other file to properly convert the data to floating-point form. (In contrast, the FF Bladed-style binary files store scaling information in the summary file.) This format also provides the maximum resolution possible in two-byte integers. Please note that, at the time of this writing, AeroDyn had not yet been updated to read these files. Continue to use the FF Bladed-style binary ".wnd" files until the release of AeroDyn 12.60 (or a later version), which will be able to read these types of files.

\section{Full-Field Bladed-Style Binary Files}

The FF Bladed-style binary files are designed to be read by both AeroDyn and GH Bladed. They have a ".wnd" extension. TurbSim normalizes the data (in the inertial reference frame coordinate system) and encodes them in 2-byte integers. The first part of the file is a header that provides Table 9. Parameters in Hub-Height Binary and
Formatted Files

\begin{tabular}{ll}
\hline Column & Description \\
\hline Time & Time from start of the simulation \\
$U$ & $U$-component wind speed \\
$u_{h}$ & Horizontal wind speed |vectorial $U+V \mid$ \\
\hline$u_{t}$ & Total wind speed |vectorial $U+V+W$ \\
$V$ & $V$-component wind speed \\
$W$ & $W$-component wind speed \\
\hline$u^{\prime}$ & Fluctuating $u$-component wind speed \\
& (the mean is removed) \\
$v^{\prime}$ & Fluctuating $v$-component wind speed \\
$w^{\prime}$ & Fluctuating $w$-component wind speed \\
\hline$u^{\prime} w^{\prime}$ & $u^{\prime} w^{\prime}$ Reynolds stress component \\
$u^{\prime} v^{\prime}$ & $u^{\prime} v^{\prime}$ Reynolds stress component \\
$v^{\prime} w^{\prime}$ & $v^{\prime} w^{\prime}$ Reynolds stress component \\
\hline TKE & Turbulent kinetic energy \\
CTKE & Coherent turbulent kinetic energy \\
\hline
\end{tabular}

Table 10. Format of Hub-Height AeroDyn Files

\begin{tabular}{ll}
\hline Column & Description \\
\hline Time & Time \\
HorSpd & $\begin{array}{l}\text { Horizontal wind speed } \\
\mid \text { vectorial } U+V \mid\end{array}$ \\
WndDir & Wind direction \\
\hline VerSpd & $\begin{array}{l}\text { Vertical wind speed }(W \\
\text { component) }\end{array}$ \\
HorShr & $\begin{array}{l}\text { Horizontal linear wind- } \\
\text { shear parameter }\end{array}$ \\
VerShr & $\begin{array}{l}\text { Vertical power-law wind- } \\
\text { shear exponent }\end{array}$ \\
\hline LnVShr & $\begin{array}{l}\text { Vertical linear wind- } \\
\text { shear parameter } \\
\text { Gust speed (not sheared } \\
\text { bstSpd }\end{array}$ \\
\hline
\end{tabular}


information about the grid; the normalized wind speeds for the NumGrid_Y $\times$ NumGrid_Z grid points follow that. See Appendix E in this guide for the file format. (The column of plots on the left side of Figure 17 shows how AeroDyn uses FF data.)

When generating these files, TurbSim adds a section to the end of the summary file that tells AeroDyn how to convert the data to floating-point form. To decode the data, AeroDyn must read both the summary file (with the ".sum" extension) and the binary FF file. TurbSim uses a newer file format than the format SNwind used. In general, this updated format retains more resolution in the normalized 2-byte integers than the previous encoding method did. A MATLAB script that reads these files is included in the TurbSim archive; it is named "TestlreadBLgrid.m."

\section{Tower Data Binary Files}

The tower data binary files are similar to the FF Bladed-style binary files, except they contain data for points in a single line at the grid center - going from the bottom of the grid to the ground - using the same vertical resolution as the rest of the grid (see Figure 4). These files have a ".twr" extension. TurbSim normalizes the data (in the inertial reference frame coordinate system) and encodes them in 2-byte integers. The first part of the file is a header that provides information about the location of the tower points and size of the file; this header is followed by the wind speeds. When generating these files, TurbSim adds a section to the end of the summary file that indicates how to convert the data to floating-point form (this is the same section that is generated for the FF Bladed-style ".wnd" binary files). See Appendix F in this guide for a more complete description of this binary format. Please note that, at the time of this writing, AeroDyn had not yet been updated to read or use these files; AeroDyn v12.60 (or later versions) will be able to read these files.

If a user requests FF binary files in TurbSim format ( $W r A D F F=$ "true"), the tower points are normalized and stored as 2-byte integers along with the full-field grid data in the file with a ".bts" extension. In that case, a separate file with the ".twr" extension is not generated.

\section{Full-Field Formatted Files}

The FF formatted files are the traditional SNLWIND-3D FF output. These three files are human readable (text), but use five times more storage than the binary files. Early versions of AeroDyn could read these files, but AeroDyn no longer supports this format. There is one file for each component, with “.u," ".v," and ".w" file extensions, respectively.

Each of the files begins with a header containing with some basic information about the simulation, and blocks of data follow. The first line in each block includes the time and the hubheight wind speed. Following that line is a table with the number of rows and columns being the number of grid points specified in the input file. The tables contain the wind speeds for the different grid points. Their orientation is as if you are looking upwind (i.e., $Y$ increases from left to right, and $Z$ increases from bottom to top), and all of the velocities are in the inertial reference frame coordinate system. A MATLAB script for reading these files is included in the TurbSim archive; it is named "TestlloadFFtxt.m." 


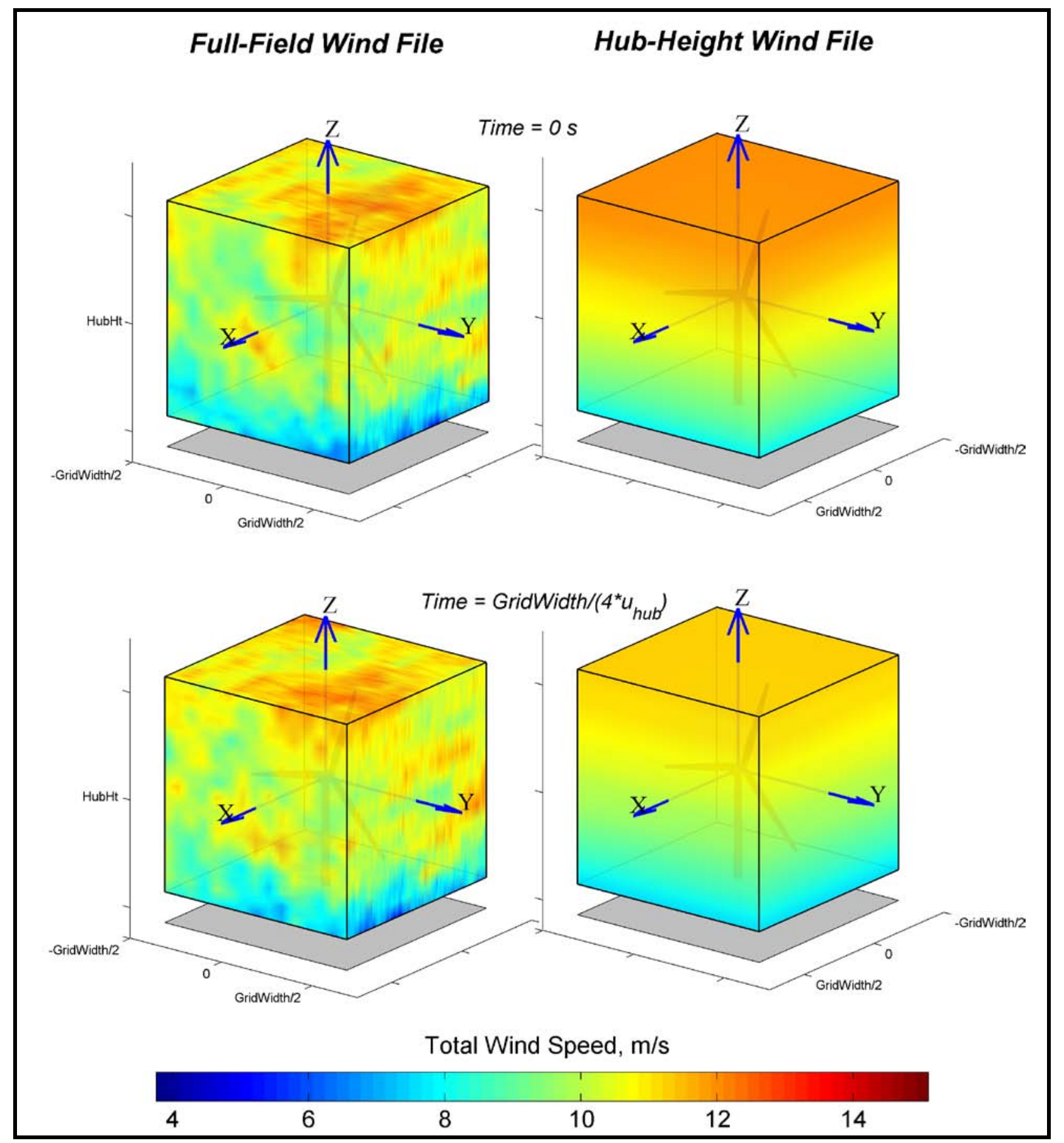

Figure 17. Example TurbSim FF and $\mathrm{HH}$ wind files as implemented in AeroDyn.

The bottom left plot shows the FF grids after marching GridWidth/4 meters (along positive $X$ ) from the position in the upper left plot; $\mathrm{HH}$ wind files (right column) do not march through the turbine. At each time step, the FF wind velocity at $X=$ GridWidth/2, $Y=0, Z=H u b H t$ is identical to the $\mathrm{HH}$ wind velocity at $X=0, Y=0, Z=H u b H t$. Because TurbSim sets the horizontal shear to 0 in the $\mathrm{HH}$ files, the velocity does not change with either $X$ or $Y$. Thus, the wind velocities in the FF and $\mathrm{HH}$ files are identical at $X=$ GridWidth/2, $Y=0, Z=H u b H t$ (where the $X$ axis on the plots emerges from the wind volume). 


\section{Coherent Turbulence Time-Step Files}

One of the unique features of TurbSim is its ability to add coherent turbulence events based on data obtained from numerical simulations of a Kelvin-Helmholtz billow. The data comes from two sources: a large-eddy simulation from NCAR and a direct numerical simulation from Colorado Research Associates (CoRA), both of Boulder, Colorado. Because the grid size of the coherent events is very large (roughly $92 \times 92$ points), these events are not added directly to the background turbulence in TurbSim. Instead, we create coherent turbulence time-step files, which have a ".cts" extension. These text files contain a header indicating how to scale the nondimensional coherent structures; the header is followed by the times and file numbers of the subset of LES or DNS data that define the coherent events. AeroDyn reads this file along with the background wind file and adds the two wind fields together. This feature can be used only in programs that use AeroDyn v12.57 or later. See the Using Coherent Turbulence Time-Step Files with AeroDyn section of this document for more information.

\section{Spectral Models}

TurbSim uses a modified version of the Sandia method [5] to generate time series based on spectral representation. Several different spectral models are available, including two IEC models, the Risø smooth-terrain model, and several NREL site-specific models (NWTCUP, GP_LLJ, WF_UPW, WF_07D, and WF_14D). This section describes the velocity spectra used in each of the models and discusses the measurements used to develop scaling for the sitespecific models. Standard deviations, $\sigma$, have been calculated by integrating the velocity spectra, $S$ :

$$
\sigma^{2}=\int_{0}^{\infty} S(f) d f .
$$

Plots comparing the velocity spectra of the different models are presented in Appendix G.

\section{IECKAI: The IEC Kaimal Model}

The IEC Kaimal model is defined in IEC 61400-1 $2^{\text {nd }}$ ed. [24], and $3^{\text {rd }}$ ed. [21] and assumes neutral atmospheric stability $\left(R_{I C H} N O=0\right) .{ }^{1}$ The spectra for the three wind components, $K=u, v, w$, are given by

$$
S_{K}(f)=\frac{4 \sigma_{K}^{2} L_{K} / \bar{u}_{h u b}}{\left(1+6 f L_{K} / \bar{u}_{h u b}\right)^{5 / 3}},
$$

where $f$ is the cyclic frequency and $L_{K}$ is an integral scale parameter. The IEC 61400-1 standard defines the integral scale parameter to be

\footnotetext{
${ }^{1}$ This model differs slightly from the original neutral spectra defined by Kaimal.
} 


$$
L_{K}= \begin{cases}8.10 \Lambda_{U}, & K=u \\ 2.70 \Lambda_{U}, & K=v, \\ 0.66 \Lambda_{U}, & K=w\end{cases}
$$

where the turbulence scale parameter, $\Lambda_{U}$, is

$$
\Lambda_{U}= \begin{cases}0.7 \cdot \min (30 \mathrm{~m}, H u b H t), & \text { Edition } 2 \\ 0.7 \cdot \min (60 \mathrm{~m}, H u b H t), & \text { Edition } 3 .\end{cases}
$$

(Note that the function $\min \left(x_{1}, x_{2}\right)$ in Eq. (23) indicates the minimum of $x_{1}$ and $x_{2}$.) The relationships between the standard deviations are defined to be

$$
\begin{aligned}
& \sigma_{v}=0.8 \sigma_{u} \\
& \sigma_{w}=0.5 \sigma_{u} .
\end{aligned}
$$

The velocity spectra (and standard deviations) of the IECKAI model are assumed to be invariant across the grid. In practice, a small amount of variation in the $u$-component standard deviation occurs due to the spatial coherence model.

\section{IECVKM: The IEC Von Karman Isotropic Model}

This IEC model is defined in IEC 61400-1 $2^{\text {nd }}$ ed. [24] for isotropic turbulence and neutral atmospheric stability. The velocity spectra for the wind components are given by

$$
S_{u}(f)=\frac{4 \sigma_{u}^{2} L / \bar{u}_{h u b}}{\left(1+71\left(f L / \bar{u}_{h u b}\right)^{2}\right)^{5 / 6}}
$$

and

$$
S_{K}(f)=\frac{2 \sigma_{K}^{2} L / \bar{u}_{h u b}}{\left(1+71\left(f L / \bar{u}_{h u b}\right)^{2}\right)^{11 / 6}}\left(1+189\left(f L / \bar{u}_{h u b}\right)^{2}\right)
$$

for $K=v, w$. In these equations, $f$ is the cyclic frequency and $L$ is an integral scale parameter. $L$ is defined using the turbulence scale parameter, $\Lambda_{U}$, from Eq. (23):

$$
L=3.5 \Lambda_{U}
$$

The IEC standard defines the relationship between the standard deviations of the components to be

$$
\sigma_{v}=\sigma_{w}=\sigma_{u} .
$$


The velocity spectra (and standard deviations) of the IECVKM model are invariant across the grid. In practice, a small amount of variation in the $u$-component standard deviation occurs due to the spatial coherence model.

\section{SMOOTH: The Risø Smooth-Terrain Model}

TurbSim also offers the Risø smooth-terrain model (SMOOTH), based on work by Højstrup et al. [28] and Olesen et al. [29]. This spectral model has separate equations for stable/neutral and for unstable flows. The SMOOTH model (as well as the site-specific models) defines the velocity spectra using local height and wind speed; this contrasts with the IEC models which use the wind speed and height of the hub to define the spectra at all points. The spectra from the SMOOTH model also form the basis for the spectra for all the site-specific models.

For stable and neutral conditions ( $\mathrm{RICH}_{-} \mathrm{NO} \geq 0$ ), the SMOOTH-model velocity spectra for the three wind components, $K$, are given by

$$
S_{K}(f)=U \operatorname{Star}^{2} \frac{S_{1, K}\left(\frac{z}{\bar{u} \phi_{M}}\right)\left(\frac{\phi_{E}}{\phi_{M}}\right)^{2 / 3}}{1.0+s_{2, K}\left(\frac{f z}{\bar{u} \phi_{M}}\right)^{5 / 3}},
$$

where $f$ is the cyclic frequency, UStar is the friction velocity input parameter, $\bar{u}$ is the mean wind speed at height $z$, and $\phi_{E}$ and $\phi_{M}$ are functions of the stability parameter, $R I C H \_N O$. The two scales, $s_{1}$ and $s_{2}$, are defined as follows for each component:

$$
\left\langle s_{1, K}, s_{2, K}\right\rangle=\left\{\begin{aligned}
\langle 79.0,263.0\rangle & K=u \\
\langle 13.0,32.0\rangle & K=v . \\
\langle 3.5, \quad 8.6\rangle & K=w
\end{aligned}\right.
$$

The theoretical standard deviations of the wind components in stable and neutral conditions are plotted in Figure 18. These values are calculated assuming infinite, continuous spectra with no spatial coherence or time-domain cross-component correlation (i.e., the input mean hub Reynolds stresses, $P C_{-} U W, P C_{-} U V$, and $P C_{-} V W$, are "none"). The standard deviations theoretically are constant across the rotor disk (using the same assumptions); in practice, however, they can appear to vary with height (depending on the input values used). This variance should decrease with increased record length. The relationships between the components' standard deviations are

$$
\begin{aligned}
& \sigma_{v}=0.76 \sigma_{u} \\
& \sigma_{w}=0.59 \sigma_{u}
\end{aligned} .
$$

For unstable flows, $\left(\mathrm{RICH}_{-} \mathrm{NO}<0\right)$, the SMOOTH spectra are modeled as the sum of lowand high-frequency spectral peaks:

$$
S(f)=S_{\text {low }}(f)+S_{\text {high }}(f) .
$$




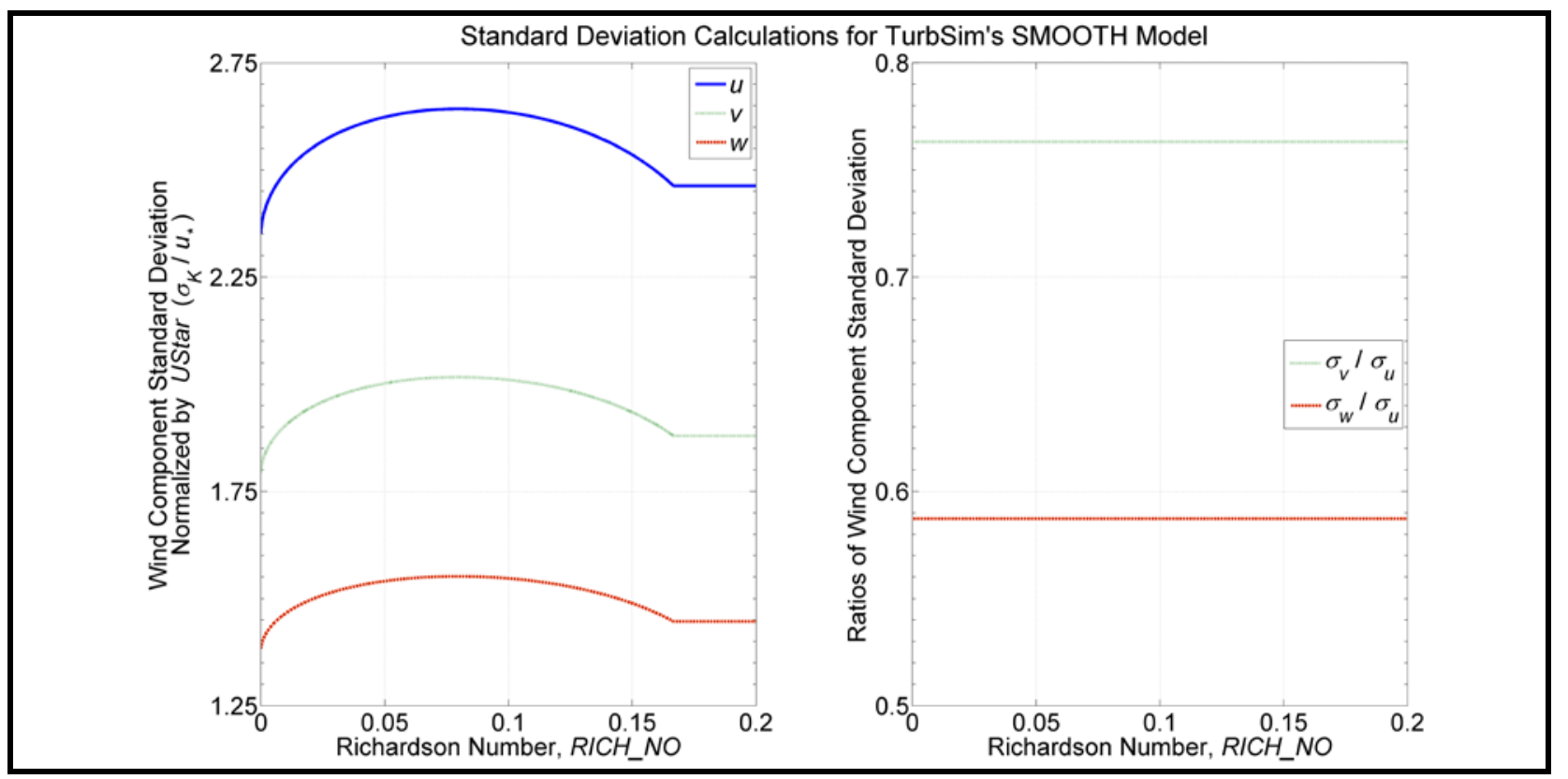

Figure 18. SMOOTH-model stable/neutral turbulence as a function of RICH_NO: left: standard deviation normalized by UStar, right: Relationships between components' standard deviations

These two peaks are defined for the three wind components as follows:

$$
\begin{gathered}
S_{u}(f)=U \operatorname{Star}^{2}\left(\frac{0.5 \frac{Z I}{\bar{u}}\left(\frac{Z I}{-L}\right)^{2 / 3}}{1+2.2\left(\frac{f Z I}{\bar{u}}\right)^{5 / 3}}+\frac{105 \frac{z}{\bar{u}\left(1+15 \frac{z}{I I}\right)}\left(1-\frac{z}{Z I}\right)^{2}}{\left(1+33 \frac{f z}{\bar{u}\left(1+15 \frac{z}{Z I}\right)}\right)^{5 / 3}\left(1+15 \frac{z}{Z I}\right)^{2 / 3}}\right), \\
S_{v}(f)=U \operatorname{Star}^{2}\left(\frac{0.95 \frac{Z I}{\bar{u}}\left(\frac{Z I}{-L}\right)^{2 / 3}}{\left(1+2 \frac{f Z I}{\bar{u}}\right)^{5 / 3}}+\frac{17 \frac{z}{\bar{u}\left(1+2.8 \frac{z}{Z I}\right)}\left(1-\frac{z}{Z I}\right)^{2}}{\left(1+9.5 \frac{f z}{\bar{u}\left(1+2.8 \frac{z}{Z I}\right)}\right)^{5 / 3}\left(1+2.8 \frac{z}{Z I}\right)^{2 / 3}}\right),
\end{gathered}
$$

and

$$
S_{w}(f)=U \operatorname{Star}^{2}\left(\frac{0.95 \frac{Z I}{\bar{u}}\left(\frac{Z I}{-L}\right)^{2 / 3}}{\left(1+2 \frac{f Z I}{\bar{u}}\right)^{5 / 3}}\left(\frac{\left(\frac{f z}{\bar{u}}\right)^{2}+\left(0.3 \frac{z}{Z I}\right)^{2}}{\left(\frac{f z}{\bar{u}}\right)^{2}+(0.15)^{2}}\right)^{1 / 2}+\frac{2 \frac{z}{\bar{u}}\left(1-\frac{z}{Z I}\right)^{2}}{1+5.3\left(\frac{f z}{\bar{u}}\right)^{5 / 3}}\right),
$$

where $f$ is the cyclic frequency, UStar and ZI are input parameters, and $\bar{u}$ is the mean wind speed at height $z$. $L$ is the Monin-Obukhov length parameter, which is a function of RICH_NO and HubHt.

The standard deviations of the wind components in unstable atmospheric conditions vary with height, the mixing layer depth $(Z I)$, and $L$. Their approximate values are determined from the following equations: 


$$
\begin{gathered}
\sigma_{u}^{2} \approx \operatorname{UStar}^{2}\left(0.62\left(\frac{Z I}{-L}\right)^{2 / 3}+4.77\left(1-\frac{z}{Z I}\right)^{2}\left(1+15 \frac{z}{Z I}\right)^{-2 / 3}\right) \\
\sigma_{v}^{2} \approx \operatorname{UStar}^{2}\left(0.71\left(\frac{Z I}{-L}\right)^{2 / 3}+2.68\left(1-\frac{z}{Z I}\right)^{2}\left(1+2.8 \frac{z}{Z I}\right)^{-2 / 3}\right) \\
\sigma_{w}^{2} \approx \operatorname{UStar}^{2}\left(0.71\left(\frac{Z I}{-L}\right)^{2 / 3}+1.46\left(1-\frac{z}{Z I}\right)^{2}\right)
\end{gathered}
$$

\section{NWTCUP: The NREL National Wind Technology Center Model}

The NWTCUP model, based on measurements from the NWTC/LIST project, represents turbulent inflow characteristics at the NWTC, downwind of a major mountain range. In this project, three towers were installed 1.5 rotor diameters upwind of the $600-\mathrm{kW}$ NWTC Advanced Research Turbine (ART). The central tower contained three-axis sonic anemometers at $15 \mathrm{~m}$, $37 \mathrm{~m}$, and $58 \mathrm{~m}$ above ground level; cup anemometers and wind vanes were located at $3 \mathrm{~m}$, $37 \mathrm{~m}$, and $58 \mathrm{~m}$; and temperature measurements were obtained at $3 \mathrm{~m}, 15 \mathrm{~m}, 37 \mathrm{~m}$, and $58 \mathrm{~m}$. Two additional towers, which were located $21 \mathrm{~m}$ north and south of the central tower, contained three-axis sonic anemometers at $37 \mathrm{~m}$. Neil Kelley et al. discuss this project and the instrumentation further [30].

The spectra for this model are based on the $40-\mathrm{Hz}$ time series data collected by the five sonic anemometers. The default spatial coherence parameters generated for this model are based on vertical coherence measured by the sonic anemometers on the central tower.

For neutral and stable flows, the NWTCUP spectra are defined by adding scaled versions of the SMOOTH-model spectra:

$$
S_{K}(f)=\sum_{i=1}^{\text {NumPeaks }_{K}} p_{i, K} S_{K, \text { SМоотH }}\left(F_{i, K} f\right),
$$

where $\mathrm{NumPeaks}_{K}=2$ for all wind components $K=u, v, w$ and the function $S_{K, \text { Sмоотн }}$ is defined in Eq. (29). All of the $p_{i, K}$ and $F_{i, K}$ scaling factors are functions of $R I C H \_N O$. Figure 19 shows the standard deviations for the three wind components and the ratios between the components' standard deviations.

For unstable flows, the NWTCUP model modifies the SMOOTH-model low- and highfrequency peaks from Eq. (33) through Eq. (35):

$$
S_{K}(f)=p_{1, K} S_{K, \text { low }, \text { Sмоотн }}\left(F_{1, K} f\right)+p_{2, K} S_{K, \text { high, Sмоотн }}\left(F_{2, K} f\right) \text {. }
$$

The scaling factors $p_{1, K}, p_{2, K}, F_{1, K}$, and $F_{2, K}$, which are empirically derived from spectra calculated using NWTC/LIST velocity measurements, are functions of the RICH_NO and UStar parameters. The standard deviations are similar to those of the unstable SMOOTH- model, scaled by the $p_{1, K}$ and $p_{2, K}$ terms. 


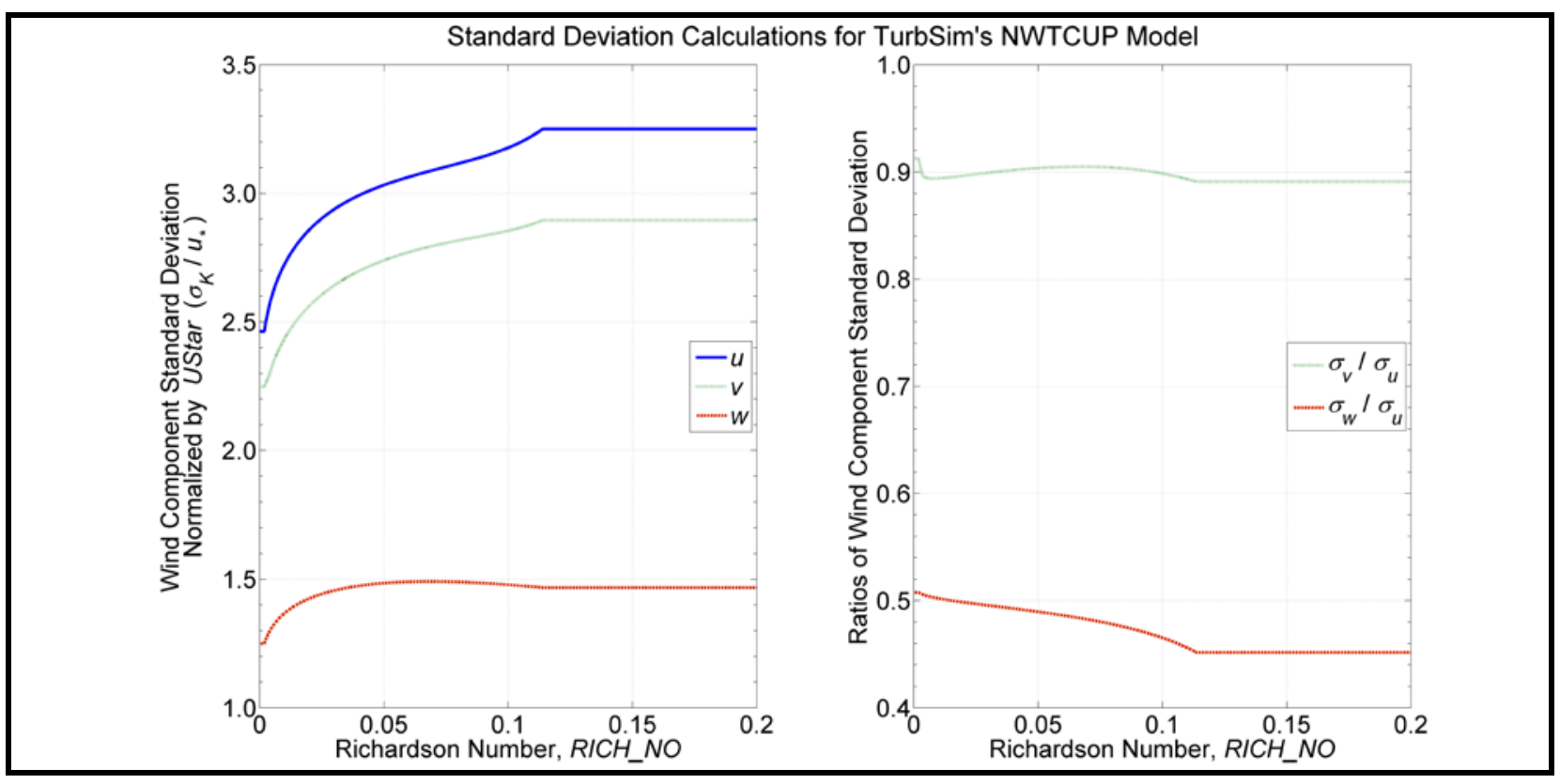

Figure 19. NWTCUP-model stable/neutral turbulence as a function of RICH_NO: left: standard deviation normalized by UStar, right: relationships between components' standard deviations

\section{GP_LLJ: The NREL Great Plains Low-Level Jet Model}

The Great Plains model (GP_LLJ) is based on measurements from a 120-m tower and from an acoustic wind profiler (SODAR [sonic detection and ranging]) obtained during the Lamar LowLevel Jet Project in southeastern Colorado. The tower included three-axis sonic anemometers at $54 \mathrm{~m}, 67 \mathrm{~m}, 85 \mathrm{~m}$, and $116 \mathrm{~m}$ above the ground; cup anemometers and direction vanes located at 3,52 , and $113 \mathrm{~m}$; and temperature measurements obtained at $3 \mathrm{~m}, 52 \mathrm{~m}, 83 \mathrm{~m}$, and $113 \mathrm{~m}$. The SODAR provided measurements of wind speed and direction at $10-\mathrm{m}$ vertical increments from $20 \mathrm{~m}$ to $500 \mathrm{~m}$. The spectra and spatial coherence parameters defined in this model are based on $20-\mathrm{Hz}$ time-series data collected at the sonic anemometers. Please refer to Neil Kelley et al. [31] for details of that project.

The GP_LLJ model defines vertical profiles of stability and of shear velocity (i.e., stability and shear velocity are functions of height). The stability profile - related to $\mathrm{RICH}_{-} \mathrm{NO}$ - is a local Monin-Obukhov stability parameter, $z L L$, and the shear velocity profile is a local $u_{*}$ value. The values used for these profiles are placed in the TurbSim summary file. Both of these profiles are calculated based on height, wind speed, and $R I C H \_N O$. The shear velocity profile also relies on UStar and $u_{*_{0}}$, which is defined in Eq. (14).

The $z / L$ and $u_{*}$ profiles are used to scale the GP_LLJ velocity spectra (in contrast, the other models use the UStar and $\mathrm{RICH}_{-} N O$ parameters, which are averaged values). For stable and neutral flows, the spectra are defined by adding peaks from the form of the SMOOTH-model spectra:

$$
S_{K}(f)=\frac{u_{*}^{2}}{U_{\text {Star }}^{2}} \sum_{i=1}^{\text {NumPeaks }_{K}} p_{i, K} S_{\text {К, Ммоотн }}\left(F_{i, K} f\right),
$$




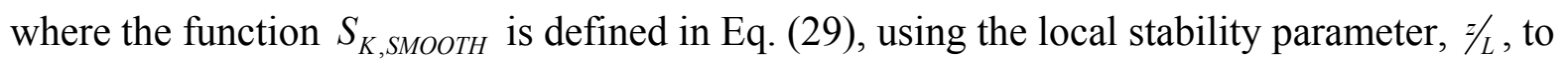
determine the values of functions $\phi_{E}$ and $\phi_{M}$ (instead of using RICH_NO as the SMOOTH model does). The $u$ and $v$ components have two peaks (NumPeaks $\left.{ }_{K}=2, K=u, v\right)$, and the $w$ component is modeled with only one peak $\left(N u m P e a k s_{w}=1\right)$. The scaling factors $p_{i, K}$ and $F_{i, K}$ are functions of both $z / L$ and $u_{*}$. The standard deviations for the three wind components are plotted in Figure 20. The ratios between the components satisfy the following inequalities:

$$
0.70 \leq \frac{\sigma_{v}}{\sigma_{u}} \leq 0.98,
$$

and

$$
0.52 \leq \frac{\sigma_{w}}{\sigma_{u}} \leq 0.71
$$

By design, most of the LLLJP data was collected in the stable atmosphere. As a result, there was not enough data to create a model of the spectra in unstable flows. Instead, the GP_LLJ spectra for unstable atmospheric conditions use the same equations as the SMOOTH model spectra in Eq. (33) through Eq. (35). The one difference is that the GP_LLJ scales the spectra using the local $u_{*}$ values instead of the UStar input parameter. The GP_LLJ spectra for unstable flows are thus defined as

$$
S_{K}(f)=\frac{u_{*}^{2}}{U S t a r^{2}} S_{K, \text { Ммоотн }}(f) \text {. }
$$

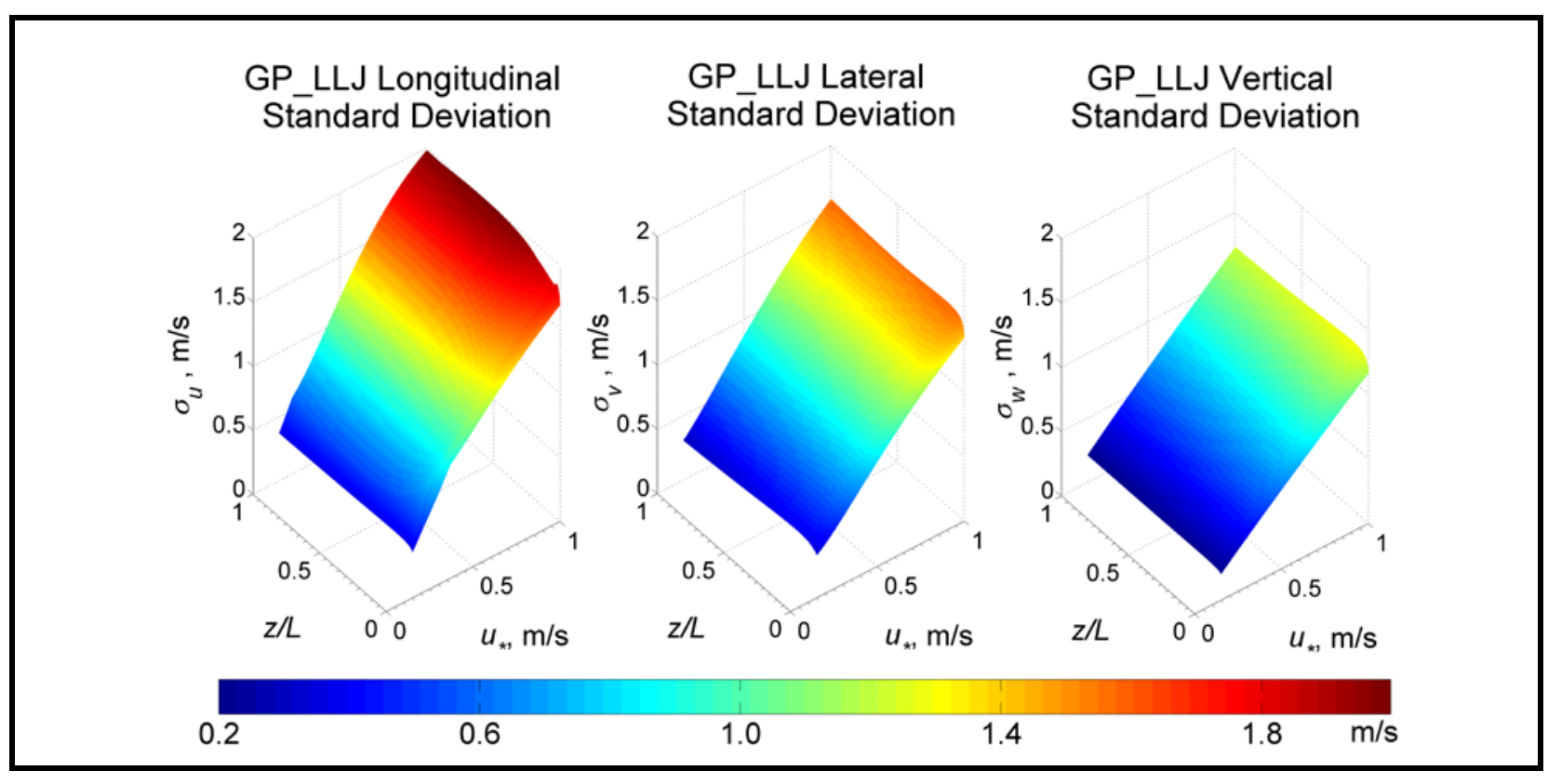

Figure 20. GPLLJ-model stable/neutral turbulence as a function of local stability and shear velocities 


\section{WF_UPW: The NREL Wind Farm, Upwind Model}

The WF_UPW wind-farm model is based on measurements taken from a 50-m tower upwind of a large wind plant in San Gorgonio Pass, California. The spectra were calculated using $50-\mathrm{Hz}$ wind-speed measurements from a three-axis sonic anemometer located $23 \mathrm{~m}$ above the ground. The parameters for spatial coherence were calculated using measurements from 5-Hz cup anemometers and direction vanes located at $5 \mathrm{~m}, 10 \mathrm{~m}, 20 \mathrm{~m}$ and $50 \mathrm{~m}$ above ground level. Please refer to Kelley [6] for details of the model development and Kelley and Wright [32] for further details on the measurements.

For neutral and stable flows, the WF_UPW spectra are defined by adding scaled versions of the SMOOTH-model spectra, using Eq. (39). All of the wind components use two spectral peaks $\left(N u m P e a k s_{K}=2, K=u, v, w\right)$ and each of the scaling factors $p_{i, K}$ and $F_{i, K}$ are functions of RICH_NO. Figure 21 shows the standard deviations for the three wind components and the ratios between the components' standard deviations.

For unstable flows, the WF_UPW model modifies the SMOOTH-model low- and highfrequency peaks, using Eq. (40). The scaling factors $p_{1, K}, p_{2, K}, F_{1, K}$, and $F_{2, K}$ are functions of the $R I C H \_N O$ parameter. The resulting standard deviations are similar to those of the unstable SMOOTH model, but scaled by the $p_{1, K}$ and $p_{2, K}$ terms.

\section{WF_14D: The NREL Wind Farm, Downwind Model (14 Rotor Diameters)}

The WF_14D wind-farm model is based on measurements taken on a 50-m tower downwind of a 41-row wind plant in San Gorgonio Pass, California. The tower was approximately 14-rotordiameters downwind of the plant, which consisted of 23-m hub-height Micon 65/13 machines with 16-m rotor diameters.

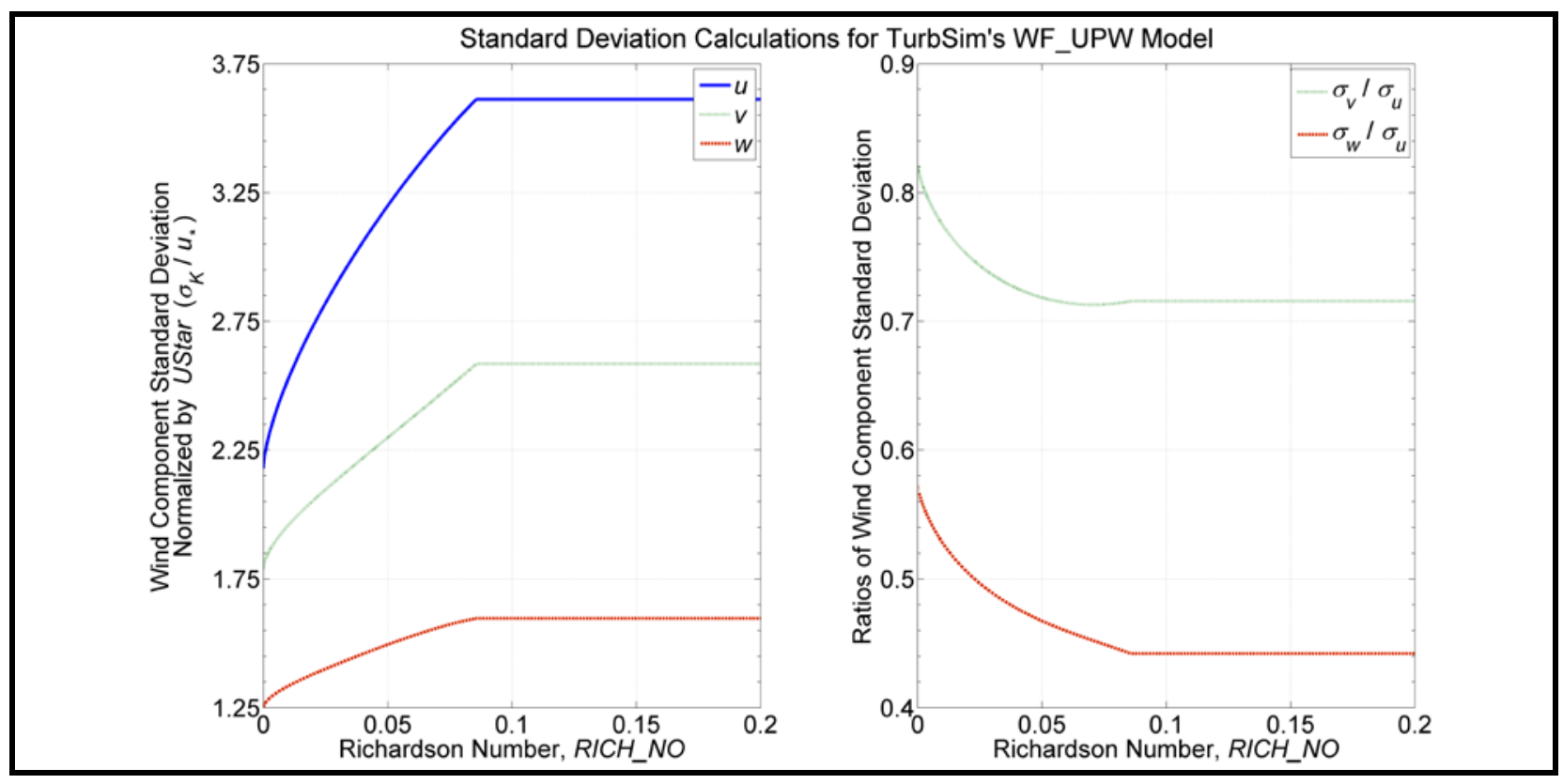

Figure 21. WF_UPW-model stable/neutral turbulence as a function of $\mathrm{RICH} N \mathrm{NO}$ : left: standard deviation normalized by UStar, right: relationships between components' standard deviations 
The spectra were calculated using $50-\mathrm{Hz}$ wind-speed measurements from a three-axis sonic anemometer located $23 \mathrm{~m}$ above the ground. The parameters for spatial coherence were calculated using measurements from $5-\mathrm{Hz}$ cup anemometers and direction vanes located at $5 \mathrm{~m}$, $10 \mathrm{~m}, 20 \mathrm{~m}$, and $50 \mathrm{~m}$ above ground. The development of this model is described by Kelley [6], and the measurements are discussed further in Kelley and Wright [32].

For neutral and stable flows, the WF_14D spectra are defined by adding scaled versions of the SMOOTH-model spectra, also using Eq. (39). All wind components use two spectral peaks $\left(\right.$ NumPeaks $\left._{K}=2, K=u, v, w\right)$ and each of the scaling factors $p_{i, K}$ and $F_{i, K}$ are functions of RICH_NO. Figure 22 shows the standard deviations for the three wind components and the ratios between the components' standard deviations.

For unstable flows, the WF_14D model modifies the SMOOTH-model low- and high-frequency peaks listed in Eq. (33) through Eq. (35):

$$
S_{K}(f)=p_{1, K} S_{K, \text { low,SМоотн }}\left(F_{1, K} f\right)+\sum_{i=2}^{\text {NumPeaks }_{K}} p_{i, K} S_{K, \text { high, Sмоотн }}\left(F_{i, K} f\right) \text {. }
$$

The $u$ - and $w$-component spectra have two peaks $\left(\right.$ NumPeaks $\left._{K}=2, K=u, w\right)$. For the $v$ component spectra, Kelley found a third peak $\left(\right.$ NumPeaks $\left._{v}=3\right)$, which he attributed to wakes from the wind turbines upstream. The scaling factors $p_{i, K}$ and $F_{i, K}, i=1,2, \ldots$, NumPeaks $_{K}$, are functions of the $R I C H \_N O$ parameter. The resulting standard deviations are similar to those of the unstable SMOOTH- -model, scaled by the $p_{i, K}$ terms.

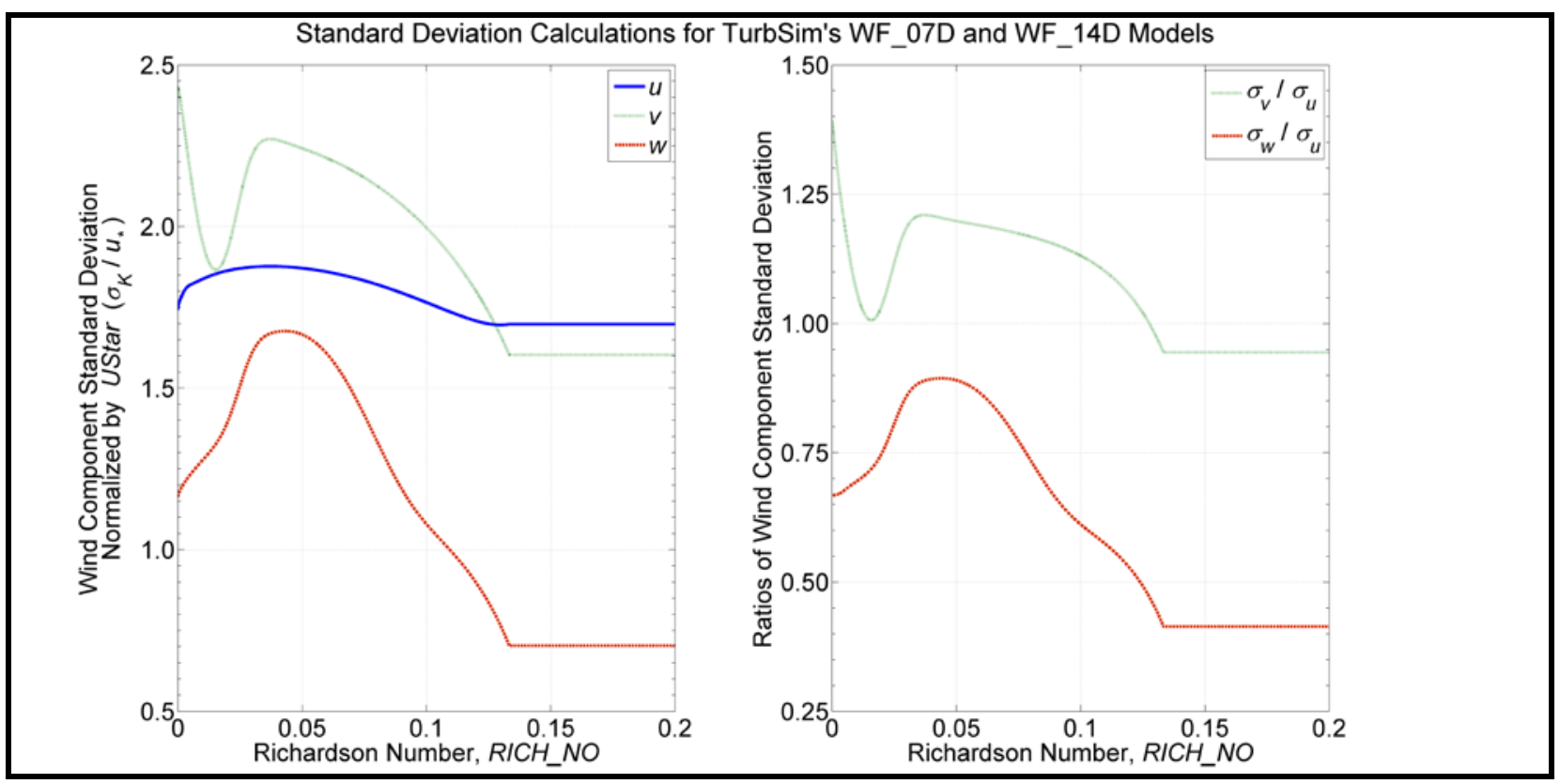

Figure 22. WF_07D- and WF_14D-model stable/neutral turbulence as a function of RICH_NO: left: standard deviation normalized by UStar, right: ratios of standard deviations 


\section{WF_07D: The NREL Wind Farm, Downwind Model (7 Rotor Diameters)}

The scaling for the WF_07D wind-farm model is based on measurements taken at row 37 of a 41-row wind plant in San Gorgonio Pass, California for the SERI Thin-Airfoil Blade Atmospheric Performance Test [33]. The 16-Hz measurements were obtained from a three-axis sonic anemometer 23-m above the ground, on a tower approximately 7-rotor-diameters downwind of a row of operating Micon 65/13 wind turbines.

These measurements were used to calculate the scaling for coherent structures and default input parameters. The measurements used to form the scaling for the WF_07D model, however, were not sufficient to develop spectral scaling or spatial coherence. As a result, the WF_07D model uses the same equations for the velocity spectra and spatial coherence as the WF $\overline{1} 4 \mathrm{D}$ model.

\section{Spatial Coherence Models}

In general, the spatial coherence between points $i$ and $j$ is defined as

$$
\operatorname{Coh}_{i, j}(f)=\frac{\left|S_{i j}(f)\right|}{\sqrt{S_{i i}(f) S_{j j}(f)}},
$$

where $f$ is the frequency, $S_{i i}$ is the power spectral density as defined in the Spectral Models section, and $S_{i j}$ is the cross-spectral density. This coherence adds correlation between the same wind components at two spatially separated points (e.g., $u_{i}-u_{j}$ correlation, not $u-v$ correlation).

The coherence functions that are implemented in TurbSim are described below.

\section{Coherence for IEC Spectral Models}

The coherence function for the $u$-component of the IEC spectral models is defined as

$$
\operatorname{Coh}_{i, j}=\exp \left(-a \sqrt{\left(\frac{f r}{\bar{u}_{h u b}}\right)^{2}+\left(0.12 \frac{r}{L_{c}}\right)^{2}}\right),
$$

where $f$ is the frequency, $r$ is the distance between points $i$ and $j$ on the grid, $a$ is the coherence decrement, $\bar{u}_{h u b}$ is the mean hub-height wind speed, and $L_{c}$ is a coherence scale parameter. For IEC 61400-1 $2^{\text {nd }}$ ed. [24], the parameters $a$ and $L_{c}$ are

$$
\begin{aligned}
a & =8.8 \\
L_{c} & =2.45 \min (30 \mathrm{~m}, H u b H t),
\end{aligned}
$$

where the function $\min (\quad)$ is the minimum of $30 \mathrm{~m}$ and HubHt. For IEC 61400-1 $3^{\text {rd }}$ ed. [21], the parameters are

$$
\begin{aligned}
a & =12 \\
L_{c} & =5.67 \min (60 \mathrm{~m}, H u b H t) .
\end{aligned}
$$


The IEC 61400-1 standard does not specify coherence for the $v$ or $w$ wind-speed components. In TurbSim, the coherence for the $v$ and $w$ components of the IEC spectral models is implemented using the identity

$$
\operatorname{Coh}_{i, j}=\left\{\begin{array}{ll}
1 & i=j \\
0 & i \neq j
\end{array} .\right.
$$

\section{Coherence for Non-IEC Spectral Models}

The coherence function for all three of the wind components, $K=u, v, w$, for the non-IEC spectral models is defined as

$$
\operatorname{Coh}_{i, j_{K}}=\exp \left(-a_{K}\left(\frac{r}{z_{m}}\right)^{\operatorname{CohExp}} \sqrt{\left(\frac{f r}{\bar{u}_{m}}\right)^{2}+\left(b_{K} r\right)^{2}}\right),
$$

where $r$ is the distance between points $i$ and $j, z_{m}$ is the mean height of the two points, and $\bar{u}_{m}$ is the mean of the wind speeds of the two points (over the entire simulation). The variables $a$ and $b$ are the input coherence decrement and offset parameter, respectively, which are defined by the values of the IncDec1, IncDec2, and IncDec3 input parameters (for each of the components). Their default values are discussed in the Input File section of this document and are plotted in Figure 13 through Figure 15.

This coherence model is based on the form suggested by Thresher et al. [34] and implemented in the IEC coherence model. The $\left(\frac{r}{z_{m}}\right)^{\text {CohExp }}$ term has been added to allow users to implement Solari's coherence definition [35]. Note that if $b=0$ and CohExp $=0$, this equation also becomes the Davenport coherence model [36].

\section{Wind Profiles}

TurbSim offers users a choice of mean wind profiles. The wind-speed profiles determine the mean $u$-component wind speeds at each height for the length of the simulation. By definition, the mean $v$ - and $w$-component wind speeds are zero. Wind-direction profiles determine the mean horizontal wind direction at each height. A wind-direction profile is calculated with the low-level jet wind-speed profile, but direction profiles are not calculated with the other speed profiles.

When computing the mean speed wind profile, TurbSim uses the inputs URef and RefHt as the reference point to calculate the mean wind speed at $H u b H t, \bar{u}_{h u b}$. The wind speeds at other heights then are calculated using $\bar{u}_{h u b}$ and $H u b H t$ as the reference point. Figure 23 shows an example of four different types of mean wind-speed profiles that were generated using default boundary conditions and $R I C H \_N O=0.05$ with the GP_LLJ model. For each of the wind-speed profiles plotted in the figure, $U R \bar{R} e f=12 \mathrm{~m} / \mathrm{s}$ and $\mathrm{RefHt}=\mathrm{HubHt}=90 \mathrm{~m}$. 


\section{Power-Law Wind Profile}

The power-law wind profile uses the PLExp input parameter to calculate the average wind speed at height $z$ using the equation

$$
\bar{u}(z)=\bar{u}\left(z_{\text {ref }}\right)\left(\frac{z}{z_{\text {ref }}}\right)^{\text {PLExp }},
$$

where $\bar{u}(z)$ is the mean wind speed at $z$ and $z_{\text {ref }}$ is a reference height above ground where the mean wind speed $\bar{u}\left(z_{\text {ref }}\right)$ is known.

\section{Logarithmic Wind Profile}

The diabatic (logarithmic) wind profile calculates the average wind speed at height $z$ using the equation

$$
\bar{u}(z)=\bar{u}\left(z_{r e f}\right) \frac{\ln (z / z 0)-\psi_{m}}{\ln \left(z_{r e f} / z 0\right)-\psi_{m}}
$$

where $\bar{u}(z)$ is the mean wind speed at $z, z_{r e f}$ is a reference height above ground where the mean wind speed is known, and $Z 0$ is the input surface roughness. The function $\psi_{m}$ varies with the $R I C H \_N O$ stability parameter. When $R I C H \_N O=0$ (as is the case with the IEC spectral models), $\psi_{m}=0$.

\section{IEC Wind Profile}

The IEC wind profile was the only wind-speed profile available in SNwind and SNLWIND-3D. This profile uses the power-law wind profile for the wind speeds at heights on the rotor disk and the logarithmic profile for heights not on the rotor disk. For example, if URef is specified at a RefHt below the rotor disk, the logarithmic profile is used to calculate the HubHt mean wind speed. Then the power-law profile would be used with the HubHt wind speed to calculate winds across the rotor disk. This profile could cause a discontinuity in the wind profile at the bottom of the rotor disk (this discontinuity would be noticed with tower points and with grids where GridWidth < GridHeight).

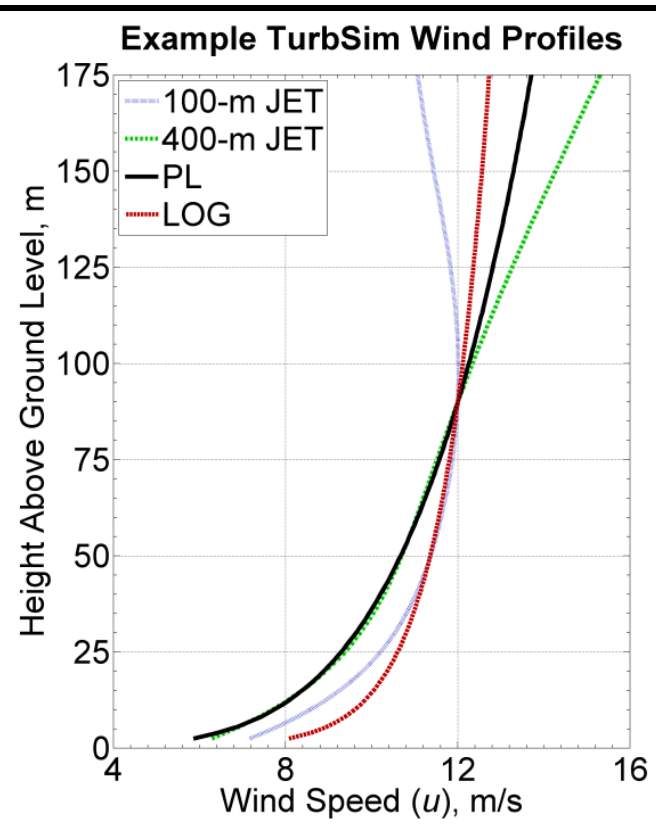

Figure 23. Example wind-speed profiles generated in TurbSim for the GP_LLJ model using a 90-m hubheight wind speed of $12 \mathrm{~m} / \mathrm{s}$, and $R / C H \_N O=0.05$ 


\section{Low-Level Jet Wind Profile}

The low-level jet wind profile is derived from LLLJP 10-minute SODAR measurements and is available with only the GP_LLJ spectral model. This profile type is unique because it generates both wind-speed and wind-direction profiles. All other wind-profile models use a constant wind direction.

The low-level jet wind-speed profile is defined using Chebyshev polynomials,

$$
\bar{u}(z)=\sum_{n=0}^{10} c_{n} \cdot T_{n}(z)
$$

where $z$ is the height above ground, $\bar{u}(z)$ is the mean wind speed at height $z, T_{n}(z)$ is the $n^{\text {th }}$ order Chebyshev polynomial, and $c_{n}$ is a Chebyshev coefficient. The Chebyshev coefficients are derived from LLLJP data and are a linear combination of the jet wind speed, $\bar{u}_{\text {ZJetMax }}$, and input parameters RICH_NO and UStar:

$$
c_{n}=C_{1, n} \bar{u}_{\text {ZJetMax }}+C_{2, n} \text { RICH_NO+C } C_{3, n} \text { UStar }+C_{4, n} .
$$

The coefficients, $C_{i, n}, i=1,2,3,4$, are determined by the input parameter ZJetMax.

The low-level jet wind-direction profile, like the wind-speed profile, is a Chebyshev polynomial with coefficients derived from the same parameters in the LLLJP data. The wind-direction profile is a relative horizontal direction and is always zero at the hub height. The HFlowAng rotation is added to the relative direction provided from this profile.

Figure 24 plots example jet wind-speed and wind-direction profiles for three different jet heights. The profiles have been generated with $\mathrm{RICH}_{-} \mathrm{NO}=0.05$, and an $80-\mathrm{m}$ (hub-height) wind speed of $12 \mathrm{~m} / \mathrm{s}$. The UStar parameter is $0.411 \mathrm{~m} / \mathrm{s}$, which is the default for these GP_LLJ conditions.

\section{Coherent Structures}

For analysis purposes, coherent structures have been defined in terms of CTKE (see Eq. (7) for the CTKE definition). A coherent structure is an event where the 3$\mathrm{s}$ mean CTKE meets a specified threshold value, determined by the mean background levels of a particular site. The event lasts from the time the threshold is first met until the 3-s mean CTKE falls below the threshold value. For the LLLJP data, the

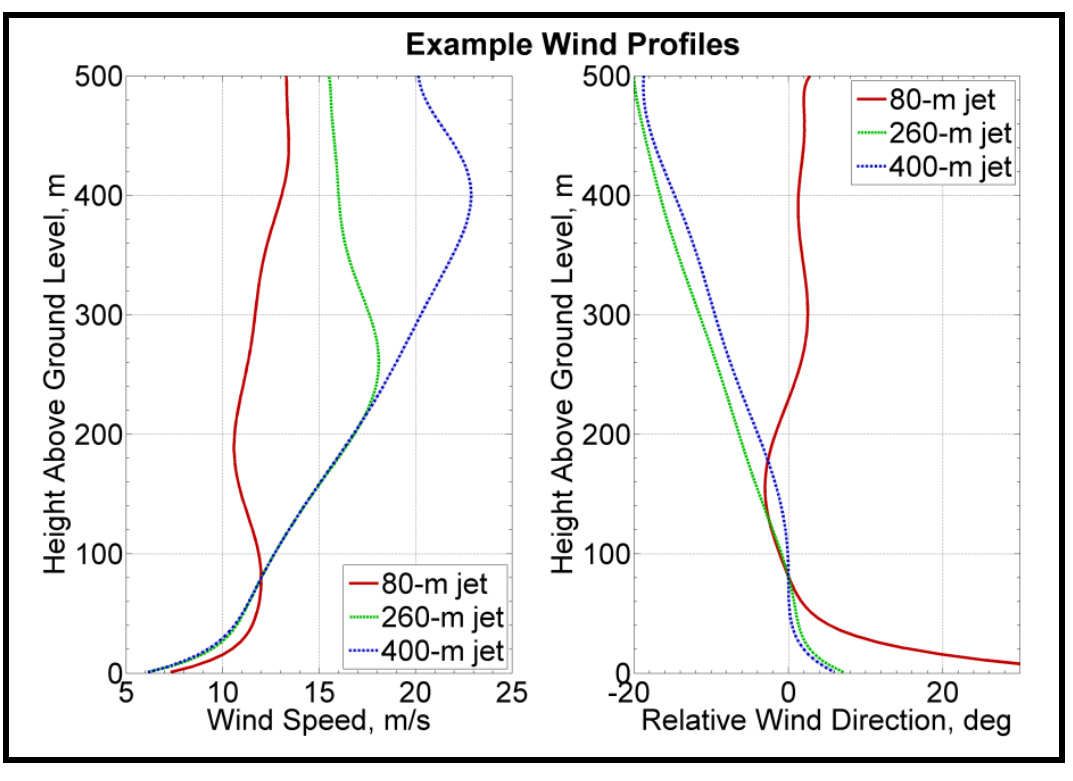

Figure 24. Example jet wind profiles with a $12 \mathrm{~m} / \mathrm{s}$ wind speed at $80 \mathrm{~m}$ and $\mathrm{RICH} \_\mathrm{NO}=0.05$ 
threshold chosen was $2 \mathrm{~m}^{2} / \mathrm{s}^{2}$, and for the LIST and wind-farm data, the threshold chosen was $5 \mathrm{~m}^{2} / \mathrm{s}^{2}$. Figure 25 gives an example of CTKE measured in the NWTC LIST experiment and shows the detected coherent structures.

The background flow that is produced in TurbSim (i.e., the wind speed data contained in the FF and $\mathrm{HH}$ output files) does contain coherent structures, using the definition above. These wind files, however, do not always generate as many coherent structures as observed in the atmosphere. To obtain more events with realistic spatial-temporal characteristics, sections (in time) of numerical simulations of a Kelvin-Helmholtz billow are added randomly to the background flow when the input parameter WrACT is "true." TurbSim generates a coherent turbulence time-step file (".cts") with the information describing how to scale the billow and where the events should be added. These events then are superimposed on the background flow in AeroDyn.

An example of the superimposed structures is shown in Figure 26. The black line in the plot shows the 3-s mean CTKE of the background flow at one point on the grid; the green line shows the 3-s mean CTKE of the background with the addition of events in the ".cts" file at the same grid point. It should be noted that the ".cts" files can decrease the CTKE of the background as well as increase it.

\section{Adding and Scaling the Coherent Structures}

The Kelvin-Helmholtz billow has been broken up into several different pieces, which are a fixed non-dimensional size with non-dimensional velocities. Before adding these pieces to the background flow, they must be scaled in space (through the DistScl input parameter) and in time to determine the dimensional velocities. TurbSim randomly chooses the start times of the billow pieces from an exponential distribution; the choice of which piece of the billow is inserted at those places is determined from a uniform random distribution.

The coherent structure scaling for the site-specific spectral models has been determined from analysis of sonic anemometer measurements at each of the respective sites, which are described in the Spectral Models section of this guide. The SMOOTH model uses the same scaling as the GP_LLJ model. Coherent structures are not added to the IEC spectral models.

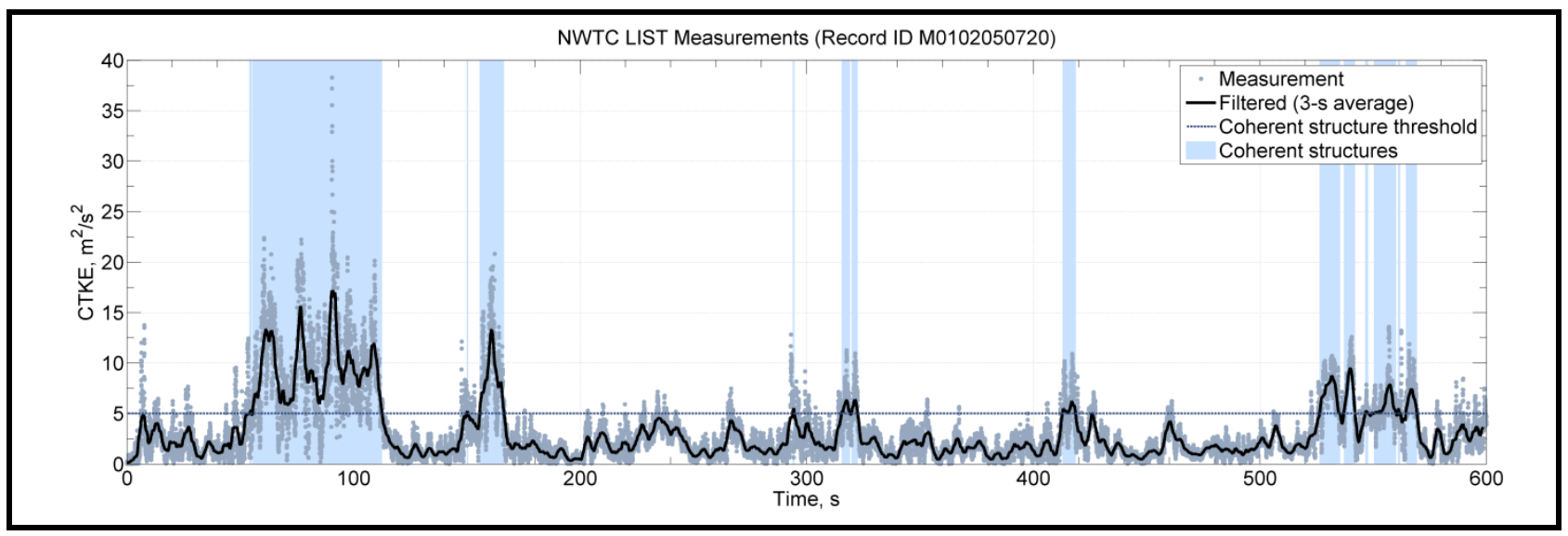

Figure 25. Coherent structures in a 10-minute period from the NWTC LIST dataset: the time series shows the 3-s mean CTKE (solid black line) crossing the dashed threshold line, which indicates the location of the coherent structures (indicated in blue) 


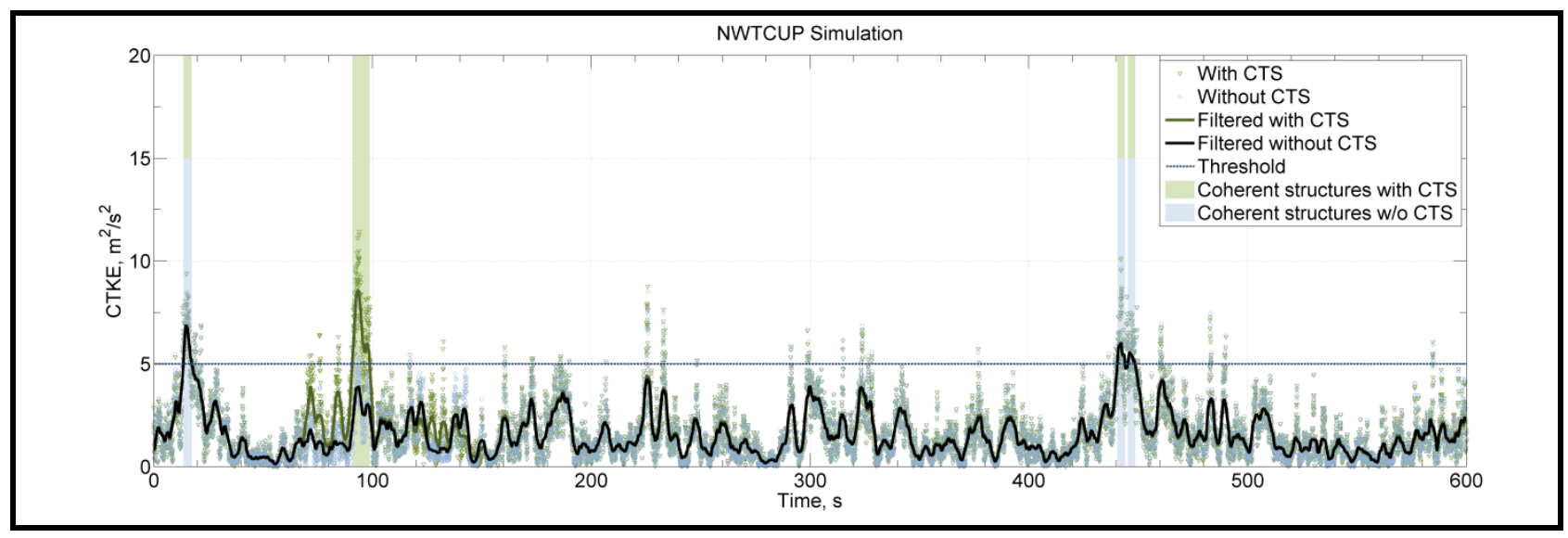

Figure 26. Example time series from the NWTCUP model: the black line indicates the background wind file; the green shows the addition of events in a coherent time-step file (".cts")

The three non-input parameters for scaling the non-dimensional pieces of the billow and adding them to the background time series are discussed below. A flow chart with these parameters is included in Appendix C.

\section{Interarrival Times}

The interarrival time is the time from the start of one event to the start of the next event. These times are exponentially distributed random variables with rate parameters determined from the analyzed datasets. For the GP_LLJ and SMOOTH models, the random distribution is influenced by the height and wind speed, $\bar{u}_{\text {center }}$, at the center of the billow. For the NWTCUP and the windfarm models, the random distribution is influenced by $\bar{u}_{\text {center }}$ and $\mathrm{RICH} N O$.

\section{Expected Length of Coherent Structures}

The length of coherent structures is the total amount of time that contains coherent structures in a given record. The expected lengths for each of the non-IEC spectral models are selected from a random distribution whose probability density function matches the data from their respective datasets.

TurbSim concatenates extra pieces of the billow to pieces that already have been added to the coherent structure file until the total length of the events is at least the expected length of the coherent structures from the datasets.

\section{Peak Coherent Turbulent Kinetic Energy}

The velocities for the coherent events are scaled to achieve a specific peak value in CTKE in the set of events added to the background. The peak CTKE is a function of several different parameters, depending on the spectral model. These parameters include height, $z$; mean wind speed of the background flow at the center of the billow, $\bar{u}_{\text {center }}$; shear across the billow (difference in mean wind speed between the top and bottom of the billow), $\Delta \bar{u}$; standard deviation of vertical wind speed at the center of the billow, $\sigma_{w_{\text {center }}}$; and input parameters RICH_NO and UStar. Some models also include a random component. Table 11 shows which particular parameters are used for each of the non-IEC spectral models. 


\section{Using Coherent Turbulence Time-Step Files with AeroDyn}

To use the coherent time-step files that TurbSim generates (files with the ".cts" extension), a coherent turbulence parameter input file must be created for AeroDyn. This file must have a ".ctp" extension, and the name of this ".ctp" file must be entered on the WindFile parameter line in the AeroDyn input file (using v12.57 or later).

See Appendix H in this document for an example of the ".ctp" input file. Do not add or delete lines from the file because AeroDyn assumes parameters are on specific lines. The parameters in the file are discussed below.

\section{CTSpath: Name of path to coherent turbulence binary data files [-]}

The CTSpath parameter is the name of the path that contains the binary data files for the coherent structures, which you must get from the coherent structure archive on the TurbSim Web site (in folder x90_i16). This directory must contain files called "Scales.les" and "Scales.dns," which contain scaling parameters for the two event types, and are used to read and convert normalized 16-bit integer binary data to real numbers. There should also be three folders in this directory, named "u," "v," and "w" respectively, containing data for the three wind components. Each of these three directories contains files named something like "u_i16_xxxxx.les."

\section{CTTSfile: Name of TurbSim CTS file [-]}

The parameter CTTSfile is the name of the coherent time-step file generated by TurbSim. It has a ".cts" extension. This file name must be specified relative to the directory from which AeroDyn will be run.

\section{CTbackgr: Name of TurbSim background FF file [-]}

The parameter CTbackgr is the name of the background turbulence file. This should be the FF wind file with the ".wnd" or ".bts" extension that was generated at the same time the ".cts" file was created. This file name also must be specified relative to the directory from which AeroDyn will be run. AeroDyn automatically looks for the ".sum" summary file that goes with a binary “.wnd" file.

Table 11. Coherent Structure Peak CTKE Scaling

\begin{tabular}{lll}
\hline TurbModel & Predictors of Peak CTKE & $\begin{array}{l}\text { Random } \\
\text { Component? }\end{array}$ \\
\cline { 2 - 3 } GP_LLJ/SMOOTH & RICH_NO, $\Delta \bar{u}, U S t a r, z$ & Yes \\
NWTCUP & $R I C H \_N O, \Delta \bar{u}, \sigma_{w_{\text {center }}}, \bar{u}_{\text {center }}$ & Yes \\
WF_UPW & $R I C H \_N O, \Delta \bar{u}, \sigma_{w_{\text {center }}}, \bar{u}_{\text {center }}$ & No \\
WF_07D & RICH_NO, $\Delta \bar{u}, \sigma_{w_{\text {center }}}$, UStar & Yes \\
WF_14D & $R I C H \_N O, \Delta \bar{u}, \sigma_{w_{\text {center }}}, \bar{u}_{\text {center }}$ & No \\
\hline
\end{tabular}




\section{$C T \_D F \_Y$ : Decimation factor in the $Y$ direction [-]}

The $C T-D F Y$ parameter is used for decimating the binary coherent turbulence data in the horizontal, $\bar{Y}$, direction. Enter the horizontal decimation factor: A value of 1 uses every point in the $Y$ direction, 2 uses every other point, etc. It is recommended that you always use the entire grid (i.e., $C T_{-} D F_{-} Y=1$ ).

\section{$C T \_D F \_Z:$ Decimation factor in the $Z$ direction [-]}

The $C T \_D F Z Z$ parameter is used for decimating the binary coherent turbulence data in the vertical, $Z$, direction. Enter the vertical decimation factor: A value of 1 uses every point in the $Z$ direction, 2 uses every other point, etc. It is recommended that you always use the entire grid (i.e., $C T \_D F \_Z=1$ ).

\section{Suggestions for Generating Coherent Turbulent Structures}

Effort has been made in TurbSim to randomize the occurrence and scaling of coherent event structures that occur in natural, nocturnal boundary layer flows. Simulations that generate coherent turbulence time-step files have up to 10 degrees of stochastic freedom-in addition to the random phases associated with each frequency at each grid point and wind component-and are designed to give some feel of the expected variability in the atmosphere. Because of the degree of variability, using more than 30 different random seeds ${ }^{2}$ for a specific set of boundary conditions is recommended.

To test the effects of a coherent structure (KH billow), we recommend using the "KHTEST" option in the IECturbc input parameter with the NWTCUP spectral model. This test function superimposes one intense coherent event in the middle of the output time series, reducing the number of stochastic degrees of freedom to no more than two (plus the random phases). The gradient Richardson number (RICH_NO) and wind shear (PLExp) of the background flow are overwritten, and TurbSim uses fixed values to scale the LES-type event. This test function is designed to generate intense turbulence, and does not necessarily reflect the variability for given boundary conditions.

The choice of the gradient Richardson number and hub wind speed largely control the impact of coherent structures on turbine response. It is recommended that at least one series of runs be made at rated wind speed and a Richardson number between 0.02 and 0.05 . Further discussion on the impact of coherent turbulent structures on wind turbines is found in [37].

\section{Warnings}

- AeroDyn v12.57 or a later version is required to read TurbSim files correctly.

- If you compile AeroDyn, you must use the compiler option "/assume:byterecl" to read the TurbSim coherent structures binary files correctly. If you use ADAMS2AD [38], be sure to use v12.17 or later so that this compiler option is set.

- Hub-height time series from HH wind files and FF wind files do not have events happening at the same time because AeroDyn shifts the FF files (see Figure 17).

\footnotetext{
${ }^{2}$ As a general rule of thumb, the number 30 is the dividing line between large and small sample statistics.
} 
- Because of the way the FFT routine works, extra time must be added to the analysis time to get the FFT to run efficiently. Due to this plus the fact that the output time could be shorter than the analysis time, the mean wind speed for the portion of the run actually used could be different from what was specified in the input file.

- The statistics calculated in the summary file are based on the complete time series generated (the analysis time plus any extra time added for the FFT calculations). Because the output time can be less than the analysis time, these statistics might differ from what can be calculated from the output files.

- Be cautious when using mean flow angle inputs with full-field grids for AeroDyn. AeroDyn marches FF grids through the turbine along the $X$-axis at the mean hub-height wind speed, regardless of the flow angles. This can give strange results if the mean flow angles are not small.

\section{Limitations}

- The GP_LLJ spectral model is estimated to be applicable up to a height of $230 \mathrm{~m}$.

- The SMOOTH spectral model and the coherent turbulence time-step files are both currently estimated to be applicable up to a height of $120 \mathrm{~m}$.

- The NWTCUP spectral model is estimated to be applicable up to heights of $120 \mathrm{~m}$.

- The wind farm spectral models (WF_UPW, WF_07D, and WF_14D) are valid only up to heights of about $50 \mathrm{~m}$.

\section{Possible Future Enhancements}

- Add options for user-defined spectra and/or user-defined coherence.

- Add the Mann model.

- Add other site-specific models if data becomes available.

\section{Caveats}

NREL makes no guarantees about the usability or accuracy of TurbSim, which is essentially a beta code. NREL does not have the resources to provide full support for this program.

\section{Disclaimer}

To view the software disclaimer, please visit the NREL Web site: http://wind.nrel.gov/designcodes/disclaimer.html. 


\section{Feedback}

If you have problems with TurbSim, please contact Bonnie Jonkman or Neil Kelley. We will respond to your needs if time and resources permit, but please do not expect an immediate response. Send comments or bug reports to:

Bonnie J. Jonkman

NWTC/3811

National Renewable Energy Laboratory

1617 Cole Blvd

Golden, CO 80401-3393

United States of America

E-mail: $\quad$ bonnie.jonkman@nrel.gov

Phone: $\quad+1$ (303) 384-6907

Fax: $\quad+1$ (303) 384-6901

Web site: http://wind.nrel.gov/designcodes 


\section{References}

[1] Laino, D. J.; Hansen, A.C. User's Guide to the Wind Turbine Dynamics Aerodynamics Computer Software AeroDyn. Salt Lake City, UT: Windward Engineering, LC, December 2002. http://wind.nrel.gov/designcodes/simulators/aerodyn/. Accessed August 25, 2009.

[2] Jonkman, J. M.; Buhl Jr., M. L. FAST User's Guide. NREL/EL-500-29798. Golden, CO: National Renewable Energy Laboratory, August 2005. http://wind.nrel.gov/designcodes/simulators/fast/. Accessed August 25, 2009.

[3] Laino, D. J.; Hansen, A.C. User's Guide to the Wind Turbine Dynamics Computer Program YawDyn. Salt Lake City, UT: Windward Engineering, LC, January 2003. http://wind.nrel.gov/designcodes/simulators/yawdyn/. Accessed August 25, 2009.

[4] MSC Software. 2005. MSC.ADAMS®. MSC.Software Corporation, Santa Ana, CA. http://www.mscsoftware.com/. Accessed August 25, 2009.

[5] Veers, P.S. Three-Dimensional Wind Simulation. SAND88-0152. Albuquerque, NM: Sandia National Laboratories, March 1988.

[6] Kelley, N.D. Full Vector (3-D) Inflow Simulation in Natural and Wind Farm Environments Using an Expanded Version of the SNLWIND (VEERS) Turbulence Code. NREL/TP-4425225. Golden, CO: National Renewable Energy Laboratory, November 1992.

[7] Bossanyi, E.A. GH Bladed Version 3.6 User Manual. Document 282/BR/010 Issue 12. Garrad Hassan and Partners Limited, 2003.

[8] Buhl, M.L. Jr. SNwind User's Guide. NREL/EL-500-30121. Golden, CO: National Renewable Energy Laboratory, October 2001.

[9] Swarztrauber, P.N. FFTPACK Version 4.1. Boulder, CO: National Center for Atmospheric Research, November 1988. http://www.scd.ucar.edu/softlib/FFTPACK.html. Accessed August 25, 2009.

[10] Kelley, N.D.; Jonkman, B.J. Overview of the TurbSim Stochastic Inflow Turbulence Simulator Version 1.21. NREL/TP-500-41137. National Renewable Energy Laboratory, Golden, CO, March 2007.

[11] Buhl, Jr. M.L. Installing NWTC Design Codes on PCs Running Windows NT®. NREL/EL500-29384. Golden, CO: National Renewable Energy Laboratory, December 2000.

[12] The MathWorks. 2008. MATLAB®. Natick, MA. http://www.mathworks.com/. Accessed August 25, 2009.

[13] Anderson, E.; Bai, Z.; Bischof, C.; Blackford, S.; Demmel, J.; Dongarra, J.; Du Croz, J.; Greenbaum, A.; Hammarling, S.; McKenney, A.; Sorensen, D. LAPACK User's Guide, $3^{\text {rd }}$ edition. Philadelphia, PA: Society for Industrial and Applied Mathematics, August 1999. http://www.netlib.org/lapack. Accessed August 25, 2009. 
[14] Lawson, C.L.; Hanson, R.J.; Kincaid, D.; Krogh, F.T. "Basic Linear Algebra Subprograms for FORTRAN usage." ACM Trans. Math. Soft., Vol. 5, September 1979; pp. 308-323.

[15] Dongarra, J.J.; Du Croz, J.; Hammarling, S.; and Hanson, R.J. “An extended set of FORTRAN Basic Linear Algebra Subprograms.” ACM Trans. Math. Soft., Vol. 14, March 1988; pp. 1-17.

[16] Meissner, Loren P. (August 1995) "ranlux.f90." http://flash.uchicago.edu/ tomek/htmls/refs/ranlux.f90.html. Accessed October 19, 2005. Copy of document on file with the author.

[17] Buhl, M.L., Jr. "NWTC Subroutine Library” NWTC Design Codes, http://wind.nrel.gov/designcodes/miscellaneous/nwtc_subs. Last modified 18-Jan-2008; Accessed August 25, 2009.

[18] L'ecuyer, P. "Efficient and Portable Combined Random Number Generators." Communications of the ACM, Vol. 31, June 1988; pp. 742-751.

[19] Lüscher, M. "A portable high-quality random number generator for lattice field theory simulations.” Computer Physics Communications, Vol. 79, February 1994; pp. 100-110.

[20] James, F. "RANLUX: A Fortran implementation of the high-quality pseudorandom number generator of Lüscher." Computer Physics Communications, Vol. 79, February 1994; pp. 111-114.

[21] IEC 61400-1 "Wind turbines-Part 1: Design requirements." $3{ }^{\text {rd }}$ edition. Geneva, Switzerland: International Electrotechnical Commission, August 2005.

[22] IEC 61400-2 "Wind turbines-Part 2: Design requirements for small wind turbines." $2^{\text {nd }}$ edition. Geneva, Switzerland: International Electrotechnical Commission, March 2006.

[23] IEC 61400-3 "Wind turbines-Part 3: Design requirements for offshore wind turbines." Draft $1^{\text {st }}$ edition. Geneva, Switzerland: International Electrotechnical Commission, January 2006.

[24] IEC 61400-1 "Wind turbine generator systems-Part 1: Safety requirements." $2^{\text {nd }}$ edition. Geneva, Switzerland: International Electrotechnical Commission, 1999.

[25] Panofsky, H.A.; Dutton, J.A. Atmospheric Turbulence: Models and Methods for Engineering Applications. New York: Wiley-Interscience, 1984. 397 pp.

[26] Dutton, J.A.; Panofsky, H.A.; Larko, D.; Shirer, H.N.; Stone, G.; Vilardo, M. Statistics of wind fluctuations over complex terrain. Report No. DOE/ET/20560-1. University Park, PA: Pennsylvania State University, Department of Meteorology, October 1979.

[27] ESDU. Report No. 85020. Characteristics of atmospheric turbulence near the ground, Part II: Single point data for strong winds (neutral atmosphere). London: Engineering Sciences Data Unit, April 1993. 
[28] Højstrup, J. "Velocity Spectra in the Unstable Planetary Boundary Layer." Journal of the Atmospheric Sciences, Vol. 39, October 1982; pp. 2239-2248.

[29] Olesen, H.R.; Larsen, S.E.; Højstrup, J. "Modeling Velocity Spectra in the Lower Part of the Planetary Boundary Layer.” Boundary-Layer Meteorology, Vol. 29, July 1984; pp. 285-312.

[30] Kelley, N.; Hand, M.; Larwood, S.; and McKenna, E. The NREL Large-Scale Turbine Inflow and Response Experiment - Preliminary Results. NREL/CP-500-30917. Golden, CO: National Renewable Energy Laboratory, January 2002.

[31] Kelley, N.D.; Shirazi, M.; Jager, D.; Wilde, S.; Adams, J.; Buhl, M.; Sullivan, P.; Patton, E. Lamar Low-Level Jet Project Interim Report. NREL/TP-500-34593. Golden, CO: National Renewable Energy Laboratory,. January 2004.

[32] Kelley, N.D.; Wright, A.D. A Comparison of Predicted and Ovserved Turbulence Wind Fields Present in Natural and Internal Wind Park Environments. NREL/TP-257-4508.

Golden, CO: National Renewable Energy Laboratory,. October 1991.

[33] Tangler, J.; Smith, B.; Jager, D.; Olsen, T. SERI Thin-Airfoil Blade Atmospheric Performance Test: Final Results NREL/TP-257-4076, Golden, CO: National Renewable Energy Laboratory, September 1990.

[34] Thresher, R.W.; Holley, W.E.; Smith, C.E.; Jafarey, N.; and Lin, S.-R. Modeling the Response of Wind Turbines to Atmospheric Turbulence. Report No. RL0/2227-81/2. Corvallis, OR: Oregon State University, Department of Mechanical Engineering, August 1981.

[35] Solari, G. "Turbulence Modeling for Gust Loading," ASCE Journal of Structural Engineering, Vol 113 (7), July 1987; pp. 1550-1569.

[36] Davenport, A.G. “The Spectrum of Horizontal Gustiness Near the Ground in High Winds,". Quarterly Journal of the Royal Meteorological Society, Vol. 87, 1961; pp. 194211.

[37] Kelley, N.D.; Jonkman, B.J.; Scott, G.N.; Bialasiewicz, J.T.; Redmond, L.S. The Impact of Coherent Turbulence on Wind Turbine Aeroelastic Response and Its Simulation.

NREL/CP-500-38074. Golden, CO: National Renewable Energy Laboratory, August 2005.

[38] Laino, D.J.; Hansen, A.C. User's Guide to the Computer Software Routines AeroDyn Interface for $A D A M S \circledR$. Salt Lake City, UT: Windward Engineering, LC, September 2001. http://wind.nrel.gov/designcodes/simulators/adams2ad/. Accessed August 25, 2009. 


\section{Appendix A: Sample TurbSim Input File}

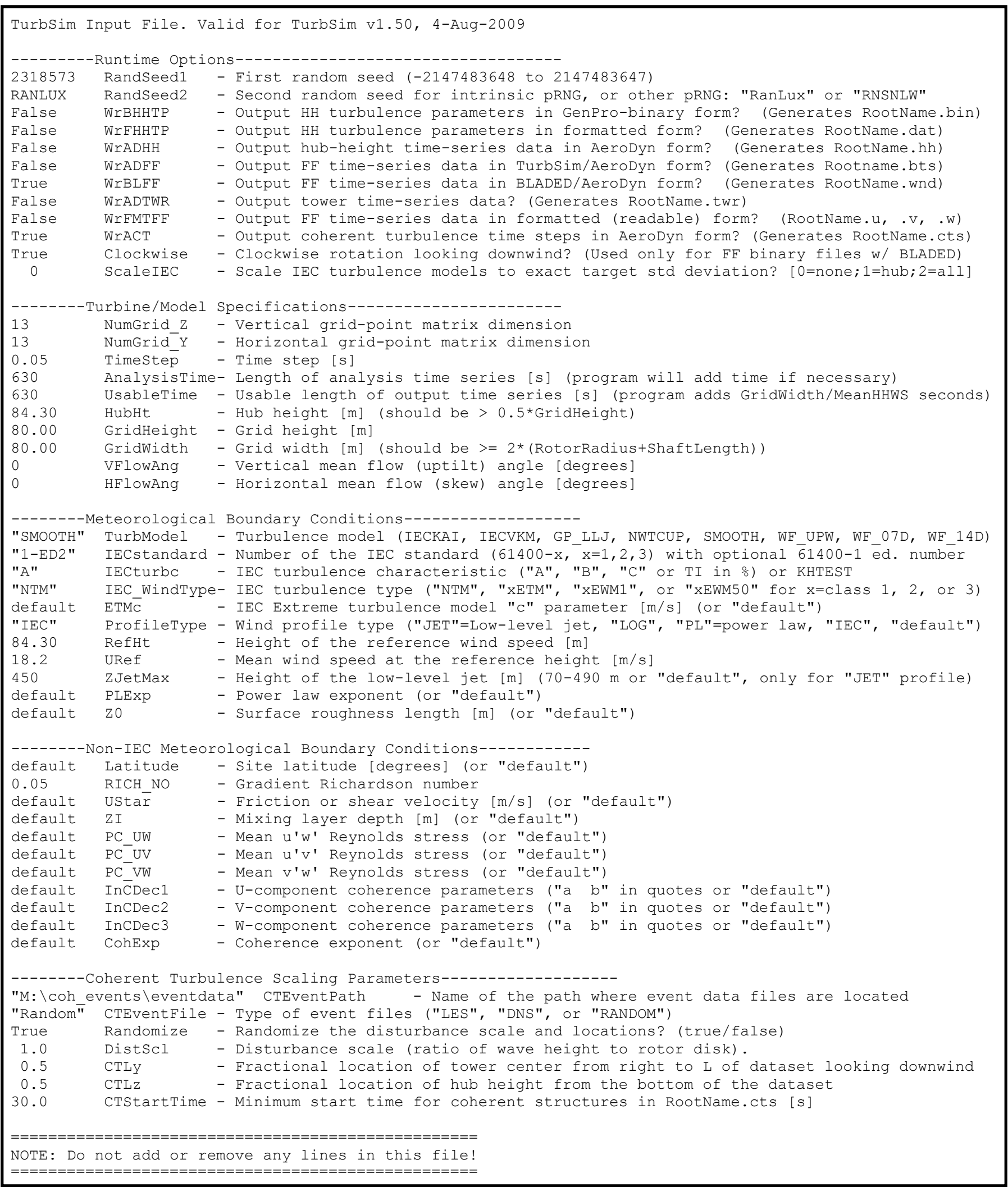

Figure A-1. Sample TurbSim input file 


\section{Appendix B: TurbSim Quick-Start Guidelines for IEC Turbulence}

To generate IEC-type turbulence, many of the parameters in the TurbSim input file can be ignored. Figure B-1 shows a TurbSim input file set up to generate IEC 61400-1 $3^{\text {rd }}$ ed., category "B" turbulence for the NTM using the Kaimal model. It creates a FF Bladed-style ".wnd" file containing 630 seconds of usable data, using a time step of $0.05 \mathrm{~s}$.

All of the unused parameters have been crossed out in Figure B-1. The parameters in black typically do not need to be changed. The input parameters that typically might have to be changed are mentioned below, along with suggestions for typical values. The Input File section of this guide describes the parameters in more detail. The parameters in blue italics in Figure B-1 should be changed based on the particular turbine for which the wind field is being generated:

- ScaleIEC: Change this parameter to the type of scaling desired. If you are unsure, use 0.

- NumGrid_Z: The number of vertical grid points should be set so there is sufficient vertical grid resolution. A typical value is an odd integer that is close to the GridHeight divided by the mean chord of the turbine's blades.

- NumGrid_Y: The number of lateral grid points should be set so there is sufficient lateral grid resolution. A typical value is an odd integer that is close to the GridWidth divided by the mean chord of the turbine's blades.

- HubHt: This is the hub height in meters of the turbine for which the turbulence is being generated.

- GridHeight: The grid height (in meters) typically is $10 \%$ larger than the turbine rotor diameter. It must be larger for turbines that have significant displacements.

- GridWidth: The grid width (in meters) typically is the same as GridHeight.

- IECturbc: The turbulence category should be "A," "B," or "C," depending on the desired 61400-1 category. Category " $\mathrm{A}$ " is the most turbulent.

- RefHt: The reference height is the height (in meters) where the input wind speed is defined. It is typically the same as HubHt.

The parameters in bold red in Figure B-1 typically are changed for each case when running design load cases:

- RandSeed 1: The random seed, which initializes the pseudo-random number generator, should be a different number for each simulation. For each case, several different seeds should be used, keeping all other input parameters constant.

- IEC_WindType: This is the wind condition for the (turbulent) IEC load cases. It often is NTM. For other conditions, see Table 5 of this guide.

- URef: This is the reference wind speed (in meters per second) at the RefHt. It typically ranges from cut-in to cut-out in $2 \mathrm{~m} / \mathrm{s}$ increments. 
Turbsim Input File. Valid for TurbSim v1.50, 4-Aug-2009

---------Runtime Options---------------------------------

1234567 RandSeed1 - First random seed (-2147483648 to 2147483647)

RANLUX RandSeed2 - Second random seed for intrinsic pRNG, or other pRNG: "RanLux" or "RNSNLW"

False WrBHHTP - Output HH turbulence parameters in GenPro-binary form? (Generates RootName.bin)

False WrFHHTP - Output HH turbulence parameters in formatted form? (Generates RootName.dat)

False WrADHH - Output hub-height time-series data in AeroDyn form? (Generates RootName.hh)

False WrADFF - Output FF time-series data in TurbSim/AeroDyn form? (Generates Rootname.bts)

True WrBLFF - Output FF time-series data in BLADED/AeroDyn form? (Generates RootName.wnd)

False WrADTWR - Output tower time-series data? (Generates RootName.twr)

False WrFMTFF - Output FF time-series data in formatted (readable) form? (RootName.u, .V, .w)

False WrACT - Output coherent turbulence time steps in AeroDyn form? (Generates RootName.cts)

True Clockwise - Clockwise rotation looking downwind? (Used only for FF binary files w/ BLADED)

o ScaleIEC - Scale IEC turbulence models to exact target std deviation? [0=none; I=hub;2=all]

--------Turbine/Model Specifications----------------------

13 Numgrid $z$ - Vertical grid-point matrix dimension

13 Numgrid_Y - Horizontal grid-point matrix dimension

0.05 Timestep - Time step [s]

630

Analysistime- Length of analysis time series [s] (program will add time if necessary)

630

84.30

UsableTime - Usable length of output time series [s] (program adds GridWidth/MeanHHWS seconds)

80.00 GridHeight

$80.00 \quad$ GridWidth

VFlowAng - Vertical mean flow (uptilt) angle [degrees]

- Hub height [m] (should be > 0.5*GridHeight)

- Grid height [m]

- Grid width [m] (should be >= 2* (RotorRadiustShaftLength))

0 HFlowAng - Horizontal mean flow (skew) angle [degrees]

-------Meteorological Boundary Conditions-----------------

"IECKAI" TurbModel - Turbulence model (IECKAI, IECVKM, GP_LLJ, NWTCUP, SMOOTH, WF_UPW, WF_07D, WF_14D)

"1-ED3" IECstandard - Number of the IEC standard (61400-x, $x=1,2,3)$ with optional $\overline{6} 1400-1$ ed. \#

"B" IECturbC - IEC turbulence characteristic ("A", "B", "C" or TI in o) or KHTEST

"NTM" IEC_WindType- IEC turbulence type ("NTM", "xETM", "xEWM1", or "xEWM50" for x=class 1, 2, or 3)

default ETMC - IEC Extreme turbulence model "C"parameter [m/s] (or "default")

"PL" ProfileType - Wind profile type ("JET"=Low-level jet, "LOG", "PL"=power law, "IEC", "default")

84.30 RefHt - Height of the reference wind speed [m]

18.2 URef - Mean wind speed at the reference height [m/s]

default ZJetMax - Height of the low-level jet [m] (70-490 m or "default", only for "JeT" profile)

default PLExp - Power law exponent (or "default")

default $\mathrm{ZO} \quad$ - Surface roughness length [m] (or "default")

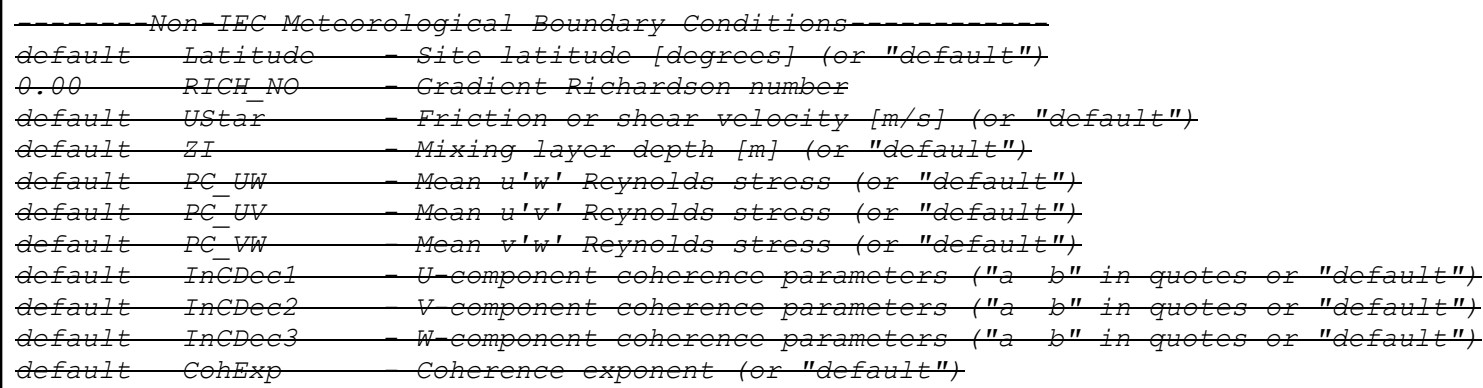

default CohExp - Coherence exponent (or "default")

"M: leventdata" CTEventPath - Name of the path where event data files are located

"Random" CTEutrile- Type of event files ("IES", "DNS", OX "RANDOM")

True Randomize - Randomize the disturbance scale and locations? (true/false)

1.0 Distsel Distubanee seale (ratio of we heighto totor disk).

0.5 CTIy - Fractional location of tower center from $R$ to $I$ of dataset looking downind

0.5 CTIz - Fractional location of hub height from the bottom of the dataset.

30.0 CTStartTime - Minimum start time for coherent structures in RootName. Cts [s]

NOTE: Do not add or remove any lines in this file!

Figure B-1. Sample TurbSim input file for IEC turbulence: parameters shown in blue should be changed based on the turbine configuration; parameters shown in red should be changed for each load case and simulation. 


\section{Appendix C: Flow Charts}

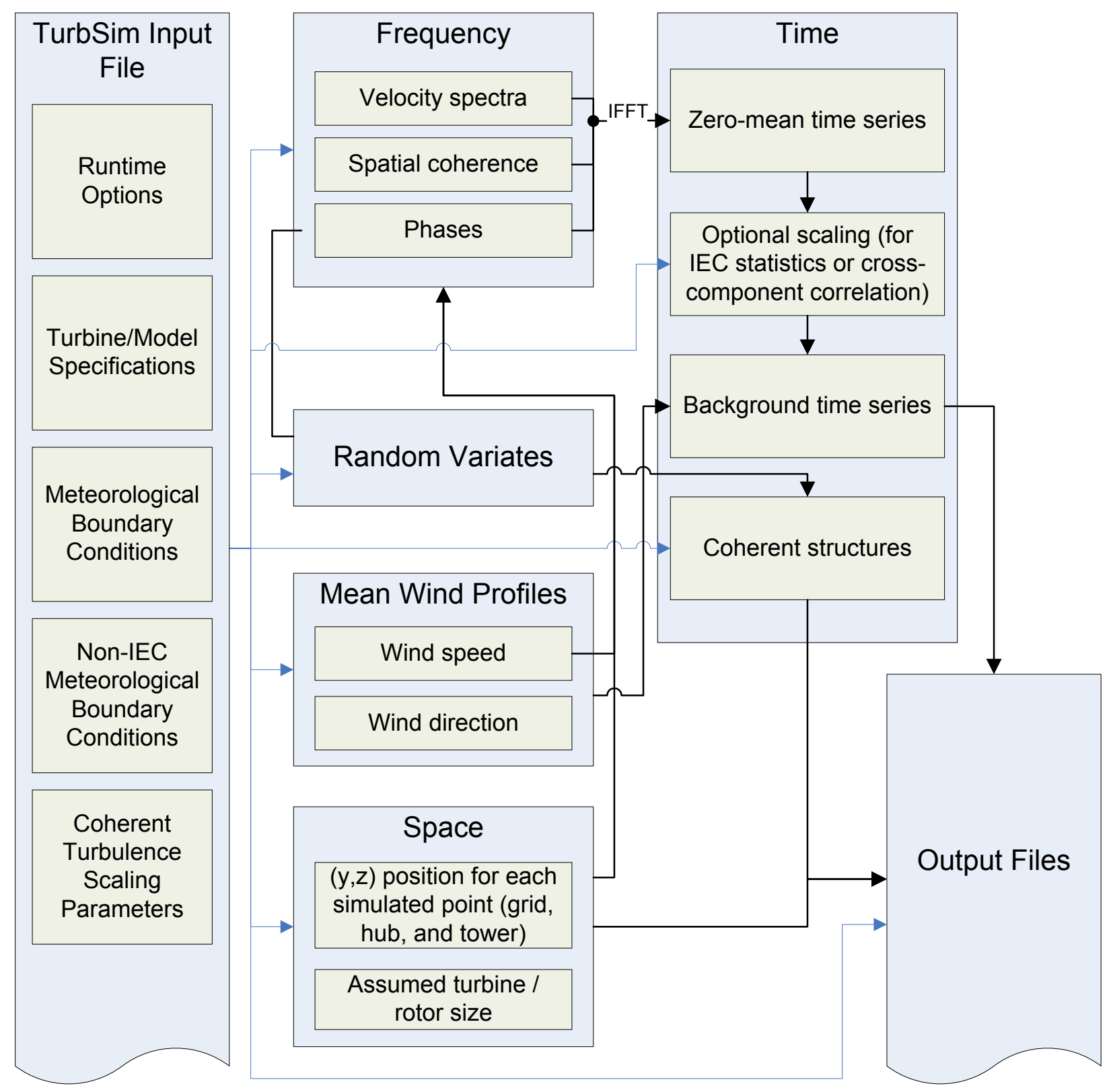

Figure C-1. Overview of the TurbSim simulation method; blue lines indicate processes influenced by input-file parameters; black lines indicate internal variables and processes 


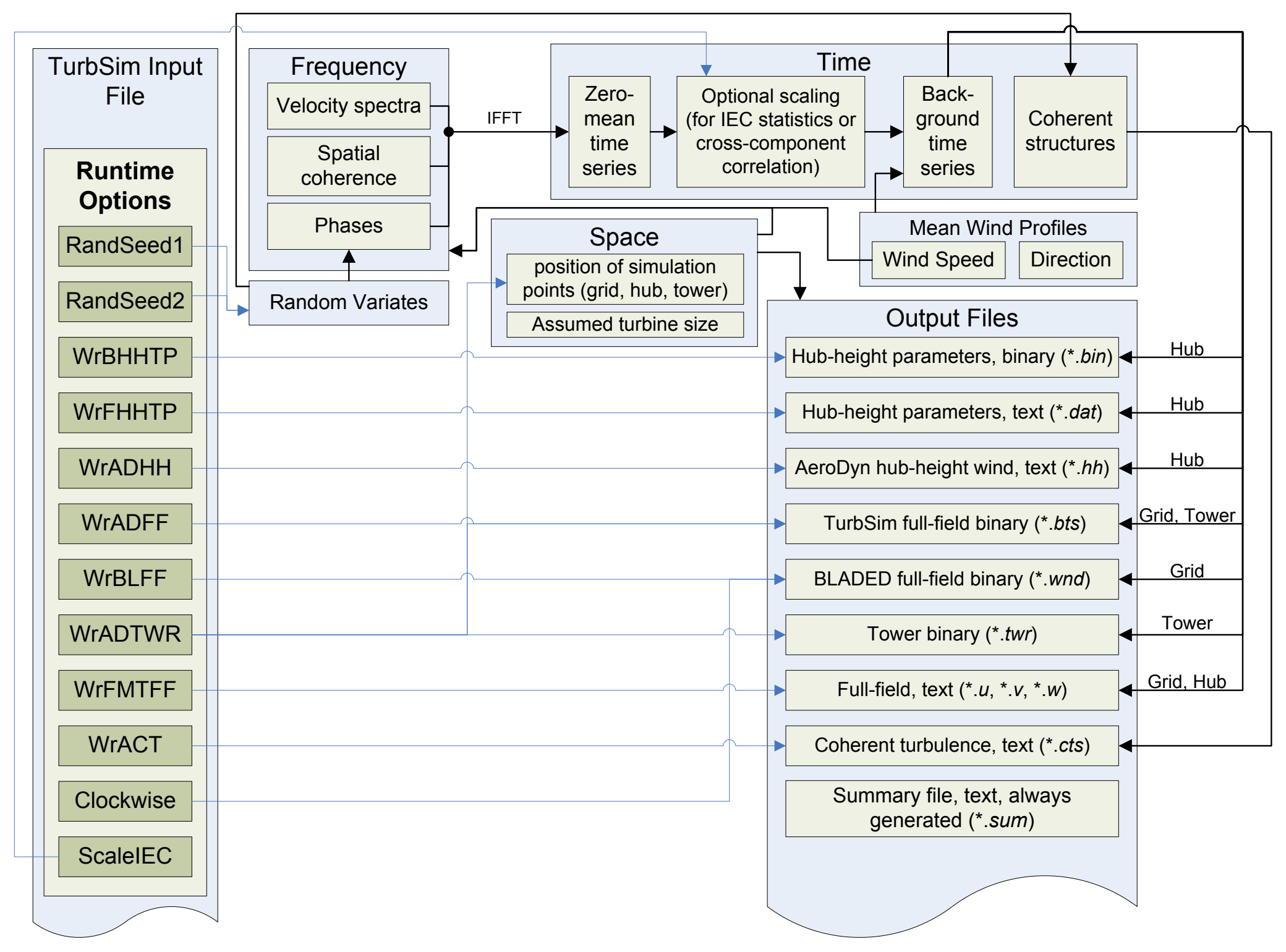

Figure C-2. Parameters in the Runtime Options section of the input file 


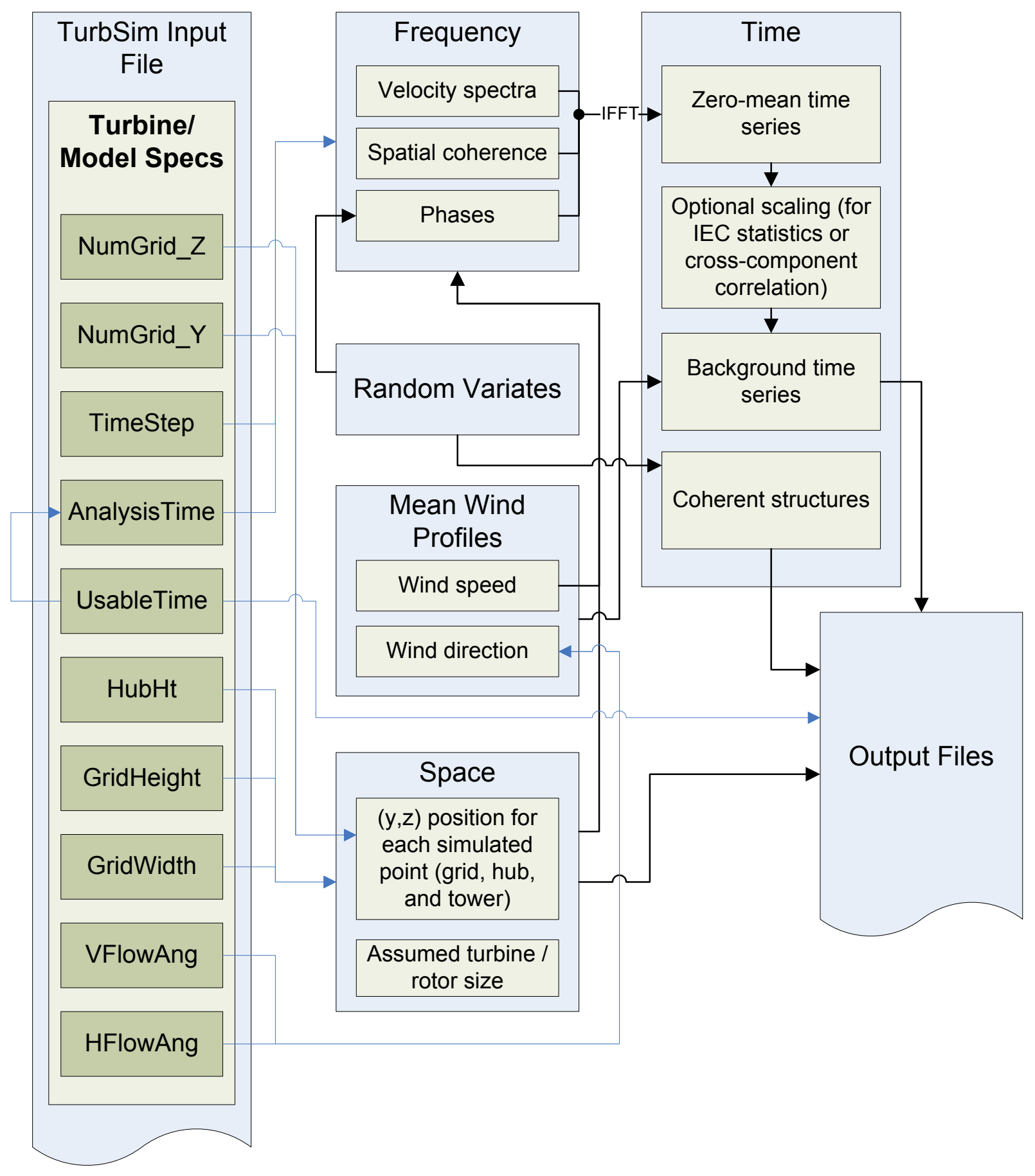

Figure C-3. Parameters in the Turbine/Model Specifications section of the TurbSim input file 


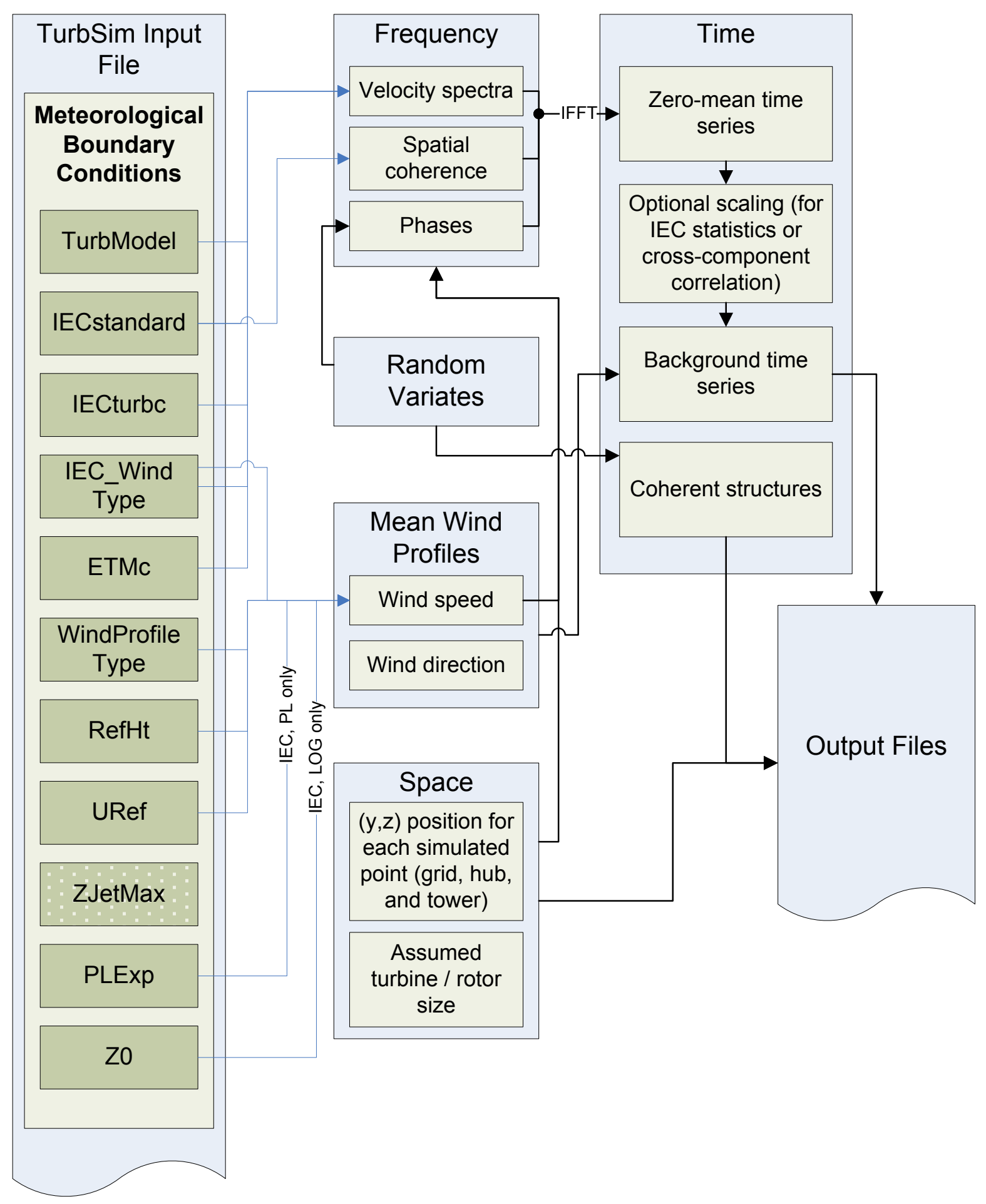

Figure C-4. Parameters in the Meteorological Boundary Conditions section of the TurbSim input file (for IECKAI and IECVKM models only) 


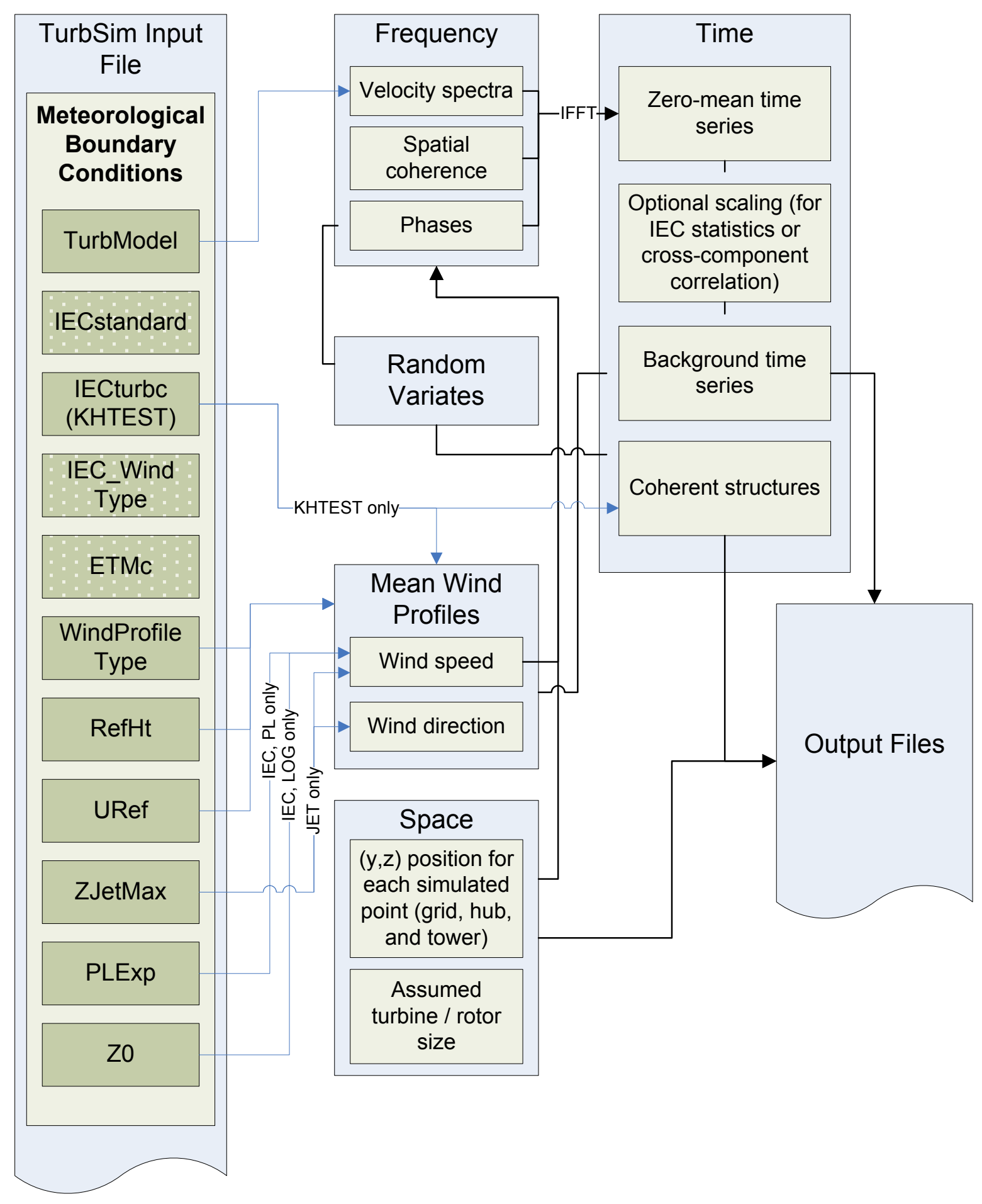

Figure C-5. Parameters in the Meteorological Boundary Conditions section of the TurbSim input file (for models other than IECKAI and IECVKM) 


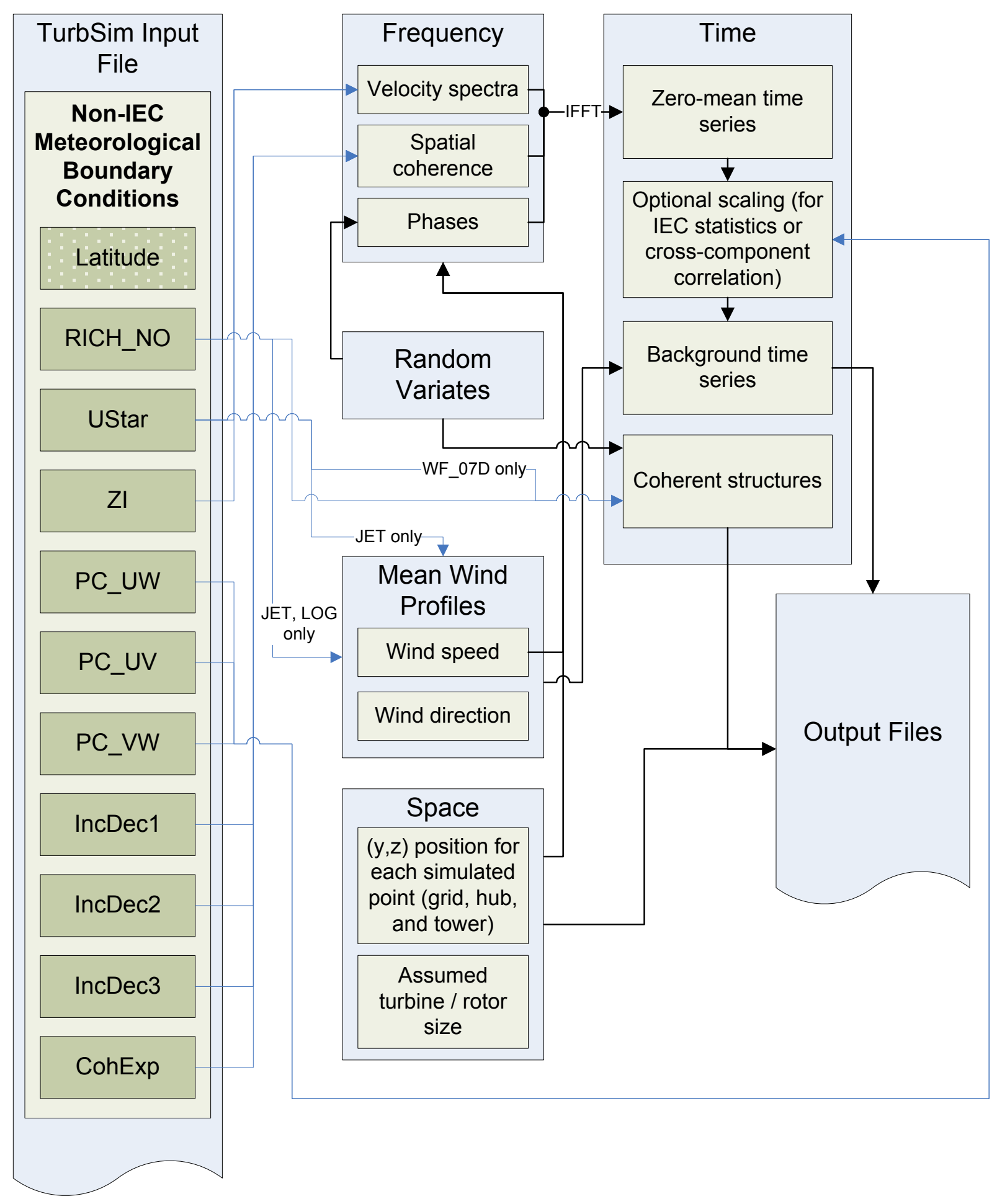

Figure C-6. Parameters in the Non-IEC Meteorological Boundary Conditions section of the TurbSim input file 


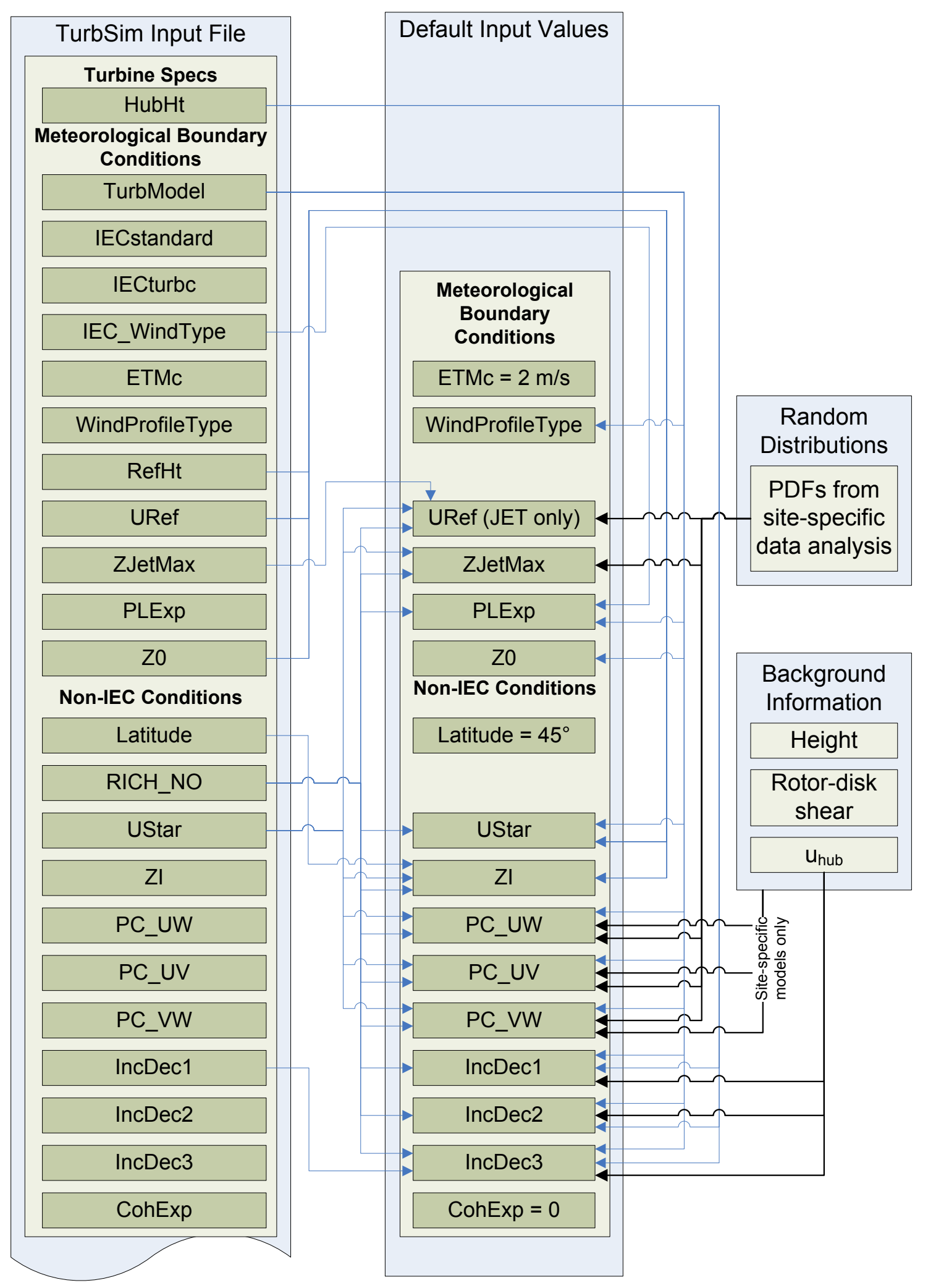

Figure C-7. Default input values for the for the Meteorological Boundary Conditions and Non-IEC Meteorological Boundary Conditions sections of the TurbSim input file 


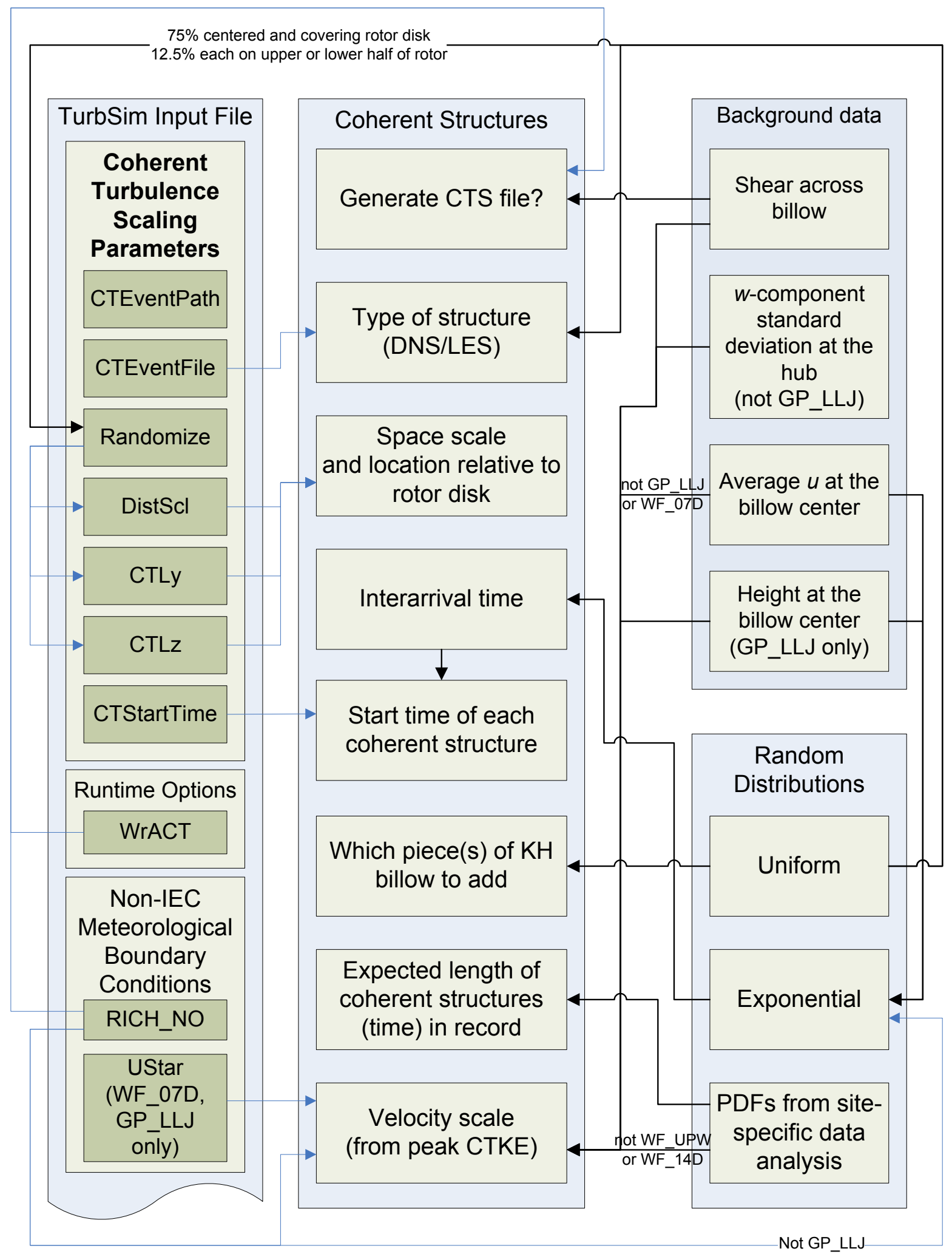

Figure C-8. Parameters for coherent structures and the Coherent Turbulence Scaling Parameters section of the TurbSim input file; the SMOOTH model uses the GP_LLJ scaling 


\section{Appendix D: Full-Field TurbSim Binary File Format}

Table D-1. Full-Field TurbSim Binary File Header Format

\begin{tabular}{|c|c|c|}
\hline Type (Bytes) & Parameter & Description \\
\hline Integer (2) & $I D$ & $\begin{array}{l}\text { Identifies the file as a TurbSim binary file. ID should } \\
\text { have the value } 7 .\end{array}$ \\
\hline Integer (4) & NumGrid_Z & The number of grid points in the vertical direction. \\
\hline Integer (4) & NumGrid_Y & The number of grid points in the horizontal direction. \\
\hline Integer (4) & $n_{\text {tower }}$ & The number of tower points below the grid. \\
\hline Integer (4) & $n_{t}$ & The number of time steps. \\
\hline Real (4) & $d z$ & $\begin{array}{l}\text { The distance in meters between two adjacent points } \\
\text { in the vertical direction. }\end{array}$ \\
\hline Real (4) & $d y$ & $\begin{array}{l}\text { The distance in meters between two adjacent points } \\
\text { in the horizontal direction. }\end{array}$ \\
\hline Real (4) & TimeStep & The time in seconds between consecutive grids. \\
\hline Real (4) & $u_{\text {hub }}$ & The mean wind speed in $\mathrm{m} / \mathrm{s}$ at hub height. \\
\hline Real (4) & HubHt & The height in meters of the hub. \\
\hline Real (4) & $Z_{\text {bottom }}$ & The height in meters of the bottom of the grid. \\
\hline \multicolumn{3}{|l|}{ for $i=1,2,3$} \\
\hline Real (4) & $V_{\text {slope }}(i)$ & $\begin{array}{l}\text { The slope used to scale the } i^{\text {th }} \text { velocity component }{ }^{3} \\
\text { from 4-byte reals into 2-byte integers. }\end{array}$ \\
\hline Real (4) & $V_{\text {intercept }}(i)$ & $\begin{array}{l}\text { The intercept used to scale the } i^{\text {th }} \text { velocity } \\
\text { component }^{3} \text { from 4-byte reals into 2-byte integers. }\end{array}$ \\
\hline \multicolumn{3}{|l|}{ end $i$} \\
\hline Integer (4) & $n_{\text {characters }}$ & $\begin{array}{l}\text { The number of characters in the ASCII string that } \\
\text { gives the TurbSim version number, date, and time } \\
\text { the file was generated. This number is no larger } \\
\text { than } 200 \text {. }\end{array}$ \\
\hline \multicolumn{3}{|c|}{ for $i=1,2, \ldots n_{\text {characters }}$} \\
\hline Integer (1) & Character $_{i}$ & $\begin{array}{l}\text { The ASCII integer representation of the } i^{\text {th }} \text { character } \\
\text { of the string that gives the TurbSim version number, } \\
\text { date, and time the file was generated. } \\
\left.\text { ACHAR(Character } r_{i}\right) \text { gives the character. }\end{array}$ \\
\hline end $i$ & & \\
\hline
\end{tabular}

${ }^{3}$ The three wind components are defined as $U=1, V=2$, and $W=3$. 
Table D-2. FF TurbSim Binary File Grid Format

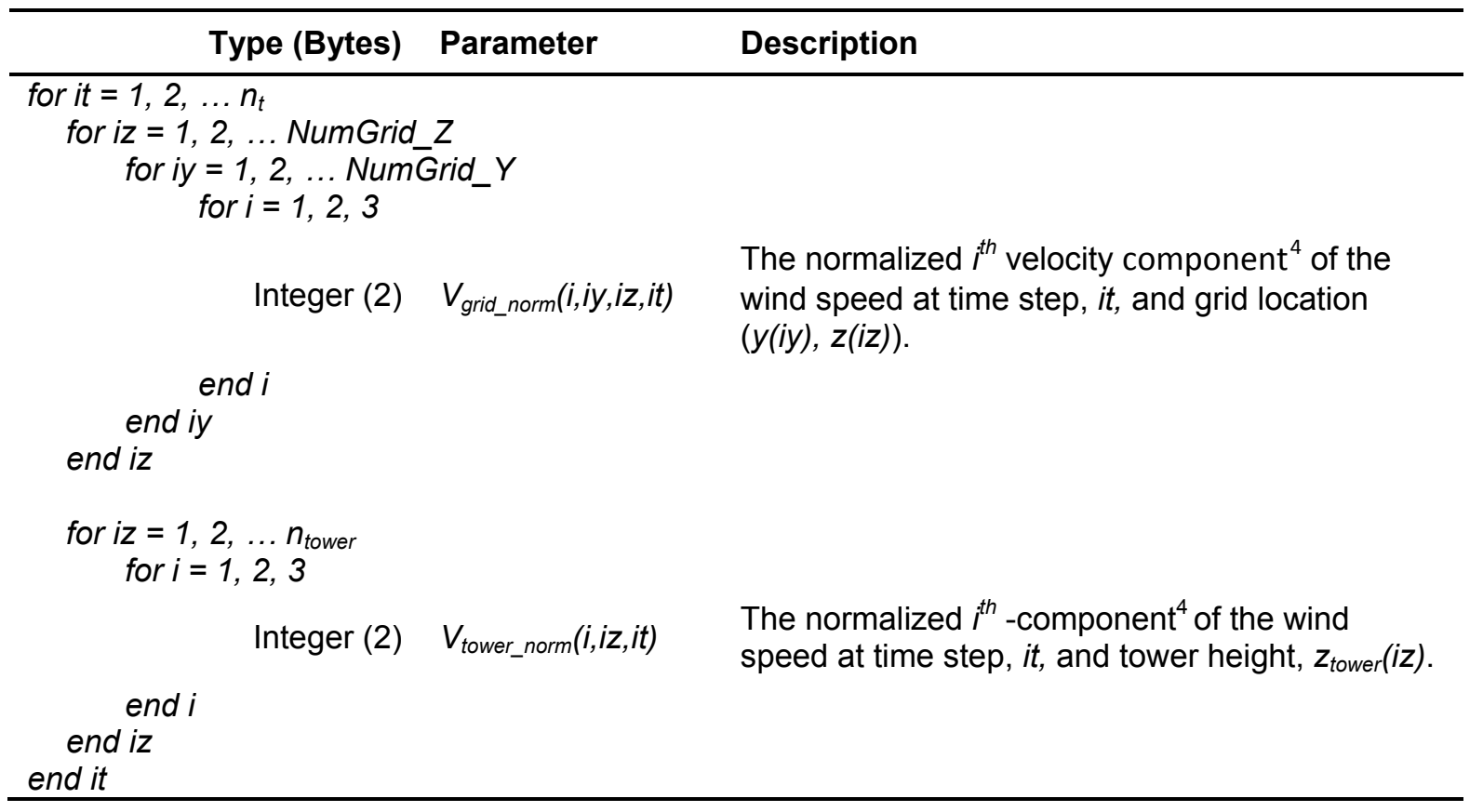

To convert the normalized wind in the FF TurbSim binary file to velocities in units of meters per second, use the following equations:

$$
\begin{gathered}
V_{\text {grid }}(i, i y, i z, i t)=\frac{V_{\text {grid_norm }}(i, i y, i z, i t)-V_{\text {intercept }}(i)}{V_{\text {slope }}(i)} \\
V_{\text {tower }}(i, i z, i t)=\frac{V_{\text {tower_norm }}(i, i z, i t)-V_{\text {intercept }}(i)}{V_{\text {slope }}(i)} .
\end{gathered}
$$

The corresponding lateral locations, $Y$, and vertical locations, $\mathrm{Z}$, of the grid and/or tower points are given in units of meters by

$$
\begin{aligned}
& Y_{\text {grid }}(\text { iy })=-0.5(\text { NumGrid_Y } Y-1) d y+(i y-1) d y \\
& Z_{\text {grid }}(i z)=Z_{\text {bottom }}+(i z-1) d z
\end{aligned}
$$

and

$$
\begin{aligned}
Y_{\text {tower }} & =0 \\
Z_{\text {tower }}(i z) & =Z_{\text {bottom }}-(i z-1) d z
\end{aligned} .
$$

${ }^{4}$ The three wind components are defined as $U=1, V=2$, and $W=3$. 


\section{Appendix E: Full-Field Bladed-Style Binary File Format}

Table E-1. Full-Field Bladed-Style Binary File Header Format

\begin{tabular}{|c|c|c|}
\hline Type (Bytes) & Parameter & Description \\
\hline Integer (2) & $I D$ & $\begin{array}{l}\text { Identifies the file as a Bladed-style binary file. ID should have } \\
\text { the value }-99 \text {. }\end{array}$ \\
\hline Integer (2) & ID2 & ID2 should have the value 4 to include the next 7 parameters. \\
\hline Integer (4) & $n c$ & The number of wind components. $n c$ should be 3 . \\
\hline Real (4) & Latitude & This value is not used in AeroDyn. \\
\hline Real (4) & $Z 0$ & The surface roughness. This value is not used in AeroDyn. \\
\hline Real (4) & Ztmp & The height at the center of the grid, in meters. \\
\hline Real (4) & $100 * T I(u)$ & The turbulence intensity of the $u$ component, in percent. \\
\hline Real (4) & $100 * \operatorname{TI}(v)$ & The turbulence intensity of the $v$ component, in percent. \\
\hline Real (4) & $100 * T I(w)$ & The turbulence intensity of the $w$ component, in percent. \\
\hline Real (4) & $d z$ & The grid spacing in the vertical direction, in meters. \\
\hline Real (4) & $d y$ & The grid spacing in the lateral direction, in meters. \\
\hline Real (4) & $u_{\text {hub }}{ }^{*}$ TimeStep & The longitudinal grid resolution, in meters. \\
\hline Integer (4) & $n t / 2$ & Half the number of points in the longitudinal direction. \\
\hline Real (4) & $u_{\text {hub }}$ & The mean wind speed (in meters per second) at hub height. \\
\hline Real (4) & Unused & The value 0 . This parameter is not used in AeroDyn. \\
\hline Real (4) & Unused & The value 0 . This parameter is not used in AeroDyn. \\
\hline Real (4) & Unused & The value 0 . This parameter is not used in AeroDyn. \\
\hline Integer (4) & Unused & The value 0 . This parameter is not used in AeroDyn. \\
\hline Integer (4) & RandSeed1 & This value is not used in AeroDyn. \\
\hline Integer (4) & NumGrid_Z & The number of grid points vertically. \\
\hline Integer (4) & NumGrid_Y & The number of grid points laterally. \\
\hline Integer (4) & Unused & The value 0 . This parameter is not used in AeroDyn. \\
\hline Integer (4) & Unused & The value 0 . This parameter is not used in AeroDyn. \\
\hline Integer (4) & Unused & The value 0 . This parameter is not used in AeroDyn. \\
\hline Integer (4) & Unused & The value 0 . This parameter is not used in AeroDyn. \\
\hline Integer (4) & Unused & The value 0 . This parameter is not used in AeroDyn. \\
\hline Integer (4) & Unused & The value 0 . This parameter is not used in AeroDyn. \\
\hline
\end{tabular}


Table E-2. Format of Grid Velocities in Full-Field Bladed-Style Binary File Format

\begin{tabular}{|c|c|c|}
\hline Type (Bytes) & Parameter & Description \\
\hline \multicolumn{3}{|c|}{$\begin{array}{l}\text { for it }=1,2, \ldots \text { nt } \\
\quad \text { for } i z=1,2, \ldots \text { NumGrid_Z } \\
\quad \text { for iy }=1,2, \ldots \text { NumGrid_Y }\end{array}$} \\
\hline Integer (2) & $U_{\text {grid_norm }}(i y, i z, i t)$ & \multirow{4}{*}{$\begin{array}{l}\text { The normalized } U \text { component of the wind speed } \\
\text { at time step, it, and grid location }(y(i y), z(i z)) \text {. } \\
\text { The normalized } V \text { component of the wind speed } \\
\text { at time step, it, and grid location }(y(i y), z(i z)) \text {. } \\
\text { The normalized } W \text { component of the wind speed } \\
\text { at time step, it, and grid location }(y(i y), z(i z)) \text {. }\end{array}$} \\
\hline Integer (2) & $V_{\text {grid_norm }}(i y, i z, i t)$ & \\
\hline Integer (2) & $W_{\text {grid_norm }}(i y, i z, i t)$ & \\
\hline $\begin{array}{l}\text { end iy } \\
\text { end iz } \\
\text { end it }\end{array}$ & & \\
\hline
\end{tabular}

To convert the normalized wind in the FF Bladed-style binary file to velocities in units of meters per second, use the following equations:

$$
\begin{gathered}
U_{\text {grid }}(i y, i z, i t)=u_{h u b}\left(\frac{T I(u)}{1000} U_{\text {grid_norm }}(i y, i z, i t)+1\right), \\
V_{\text {grid }}(i y, i z, i t)=u_{h u b}\left(\frac{T I(v)}{1000} V_{\text {grid_norm }}(i y, i z, i t)\right),
\end{gathered}
$$

and

$$
W_{\text {grid }}(i y, i z, i t)=u_{\text {hub }}\left(\frac{T I(w)}{1000} W_{\text {grid_norm }}(i y, i z, i t)\right) .
$$

Here $T I$ represents the turbulence intensity as a decimal, not a percentage.

The corresponding vertical locations, $Z$, of the grid points are given in units of meters by

$$
Z_{\text {grid }}(i z)=-0.5(\text { NumGrid_Z } Z-1) d z+\text { HubHt }- \text { HeightOffset }+(i z-1) d z
$$

using values of $\mathrm{HubHt}$ and HeightOffset from the summary file. The lateral locations, $Y$, of the grid points depend on the input value Clockwise (read from the summary file) and are given by

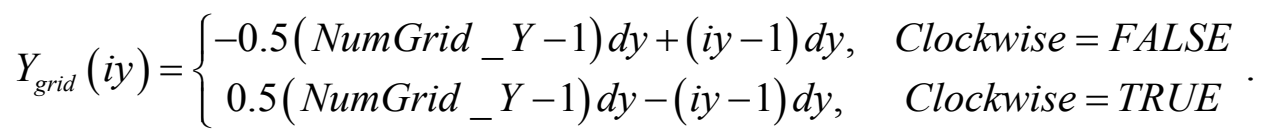




\section{Appendix F: Tower Data Binary File Format}

TurbSim tower files have a ".twr" extension. Each file contains a header of 4-byte real and integer values, followed by 2-byte integer time series of the three wind components at each point on the tower grid. The wind components at the tower points are normalized and stored in 2-byte binary integers, exactly the same way that Bladed-style full-field wind files are written. The tower files have the same vertical resolution as the full-field grid, with points going downward from the bottom of the full grid in a single line at the tower centerline.

Table F-1: Format of Header in TurbSim Binary Tower-Data File

\begin{tabular}{|c|c|c|}
\hline $\begin{array}{l}\text { Type } \\
\text { (Bytes) }\end{array}$ & Parameter & Description \\
\hline Real (4) & $d z$ & Vertical grid resolution, in meters. \\
\hline Real (4) & $u_{\text {hub }}{ }^{*}$ TimeStep & Longitudinal grid resolution, in meters. \\
\hline Real (4) & $Z \max$ & The height of the highest tower point, in meters. \\
\hline Real (4) & $n t$ & The number of points in the longitudinal direction. \\
\hline Real (4) & $n z$ & The number of vertical tower points. \\
\hline Real (4) & $u_{\text {hub }}$ & The mean wind speed, in meters per second. \\
\hline Real (4) & $100 * T I(u)$ & The turbulence intensity of the $u$ component, in percent. \\
\hline Real (4) & $100 * T I(v)$ & The turbulence intensity of the $v$ component, in percent. \\
\hline Real (4) & $100 * T I(w)$ & The turbulence intensity of the $w$ component, in percent. \\
\hline
\end{tabular}

Table F-2: Format of Grid Velocities in TurbSim Binary Tower-Data File

For each increasing time step ( $n t$ points),and starting at the top of the grid, going downward ( $n z$ points) the data are stored as:

\begin{tabular}{|c|c|c|}
\hline Type (Bytes) & Parameter & Description \\
\hline \multicolumn{3}{|l|}{$\begin{array}{l}\text { for it }=1,2, \ldots n t \\
\quad \text { for } i z=1,2, \ldots n z\end{array}$} \\
\hline Integer (2) & $U_{\text {tower_norm }}(i z, i t)$ & $\begin{array}{l}\text { Normalized } U \text { component of the wind speed at } \\
\text { time step, it, and height } z \text { (iz). }\end{array}$ \\
\hline Integer (2) & $V_{\text {tower_norm }}(i z, i t)$ & $\begin{array}{l}\text { Normalized } V \text { component of the wind speed at } \\
\text { time step, it, and height } z \text { (iz). }\end{array}$ \\
\hline Integer (2) & $W_{\text {tower_norm }}(i z, i t)$ & $\begin{array}{l}\text { Normalized } W \text { component of the wind speed } \\
\text { at time step, it, and height } z(i z) \text {. }\end{array}$ \\
\hline $\begin{array}{l}\text { end iz } \\
\text { end it }\end{array}$ & & \\
\hline
\end{tabular}


To convert the normalized wind in the tower data binary file to velocities in units of meters per second, use the following equations:

$$
\begin{gathered}
U_{\text {tower }}(i z, i t)=u_{\text {hub }}\left(\frac{T I(u)}{1000} U_{\text {tower_norm }}(i z, i t)+1\right) \\
V_{\text {tower }}(i z, i t)=u_{\text {hub }}\left(\frac{T I(v)}{1000} V_{\text {tower_norm }}(i z, i t)\right)
\end{gathered}
$$

and

$$
W_{\text {tower }}(i z, i t)=u_{\text {hub }}\left(\frac{T I(w)}{1000} W_{\text {tower_norm }_{\text {no }}}(i z, i t)\right) .
$$

Here $T I$ represents the turbulence intensity as a decimal, not a percentage.

The corresponding lateral locations, $Y$, and vertical locations, $Z$, of the tower points are given in units of meters using values of Zmax from the file header:

$$
\begin{aligned}
Y_{\text {tower }} & =0 \\
Z_{\text {tower }}(i z) & =Z_{\text {max }}-(i z-1) d z .
\end{aligned}
$$




\section{Appendix G: Velocity Spectra Comparison Plots}
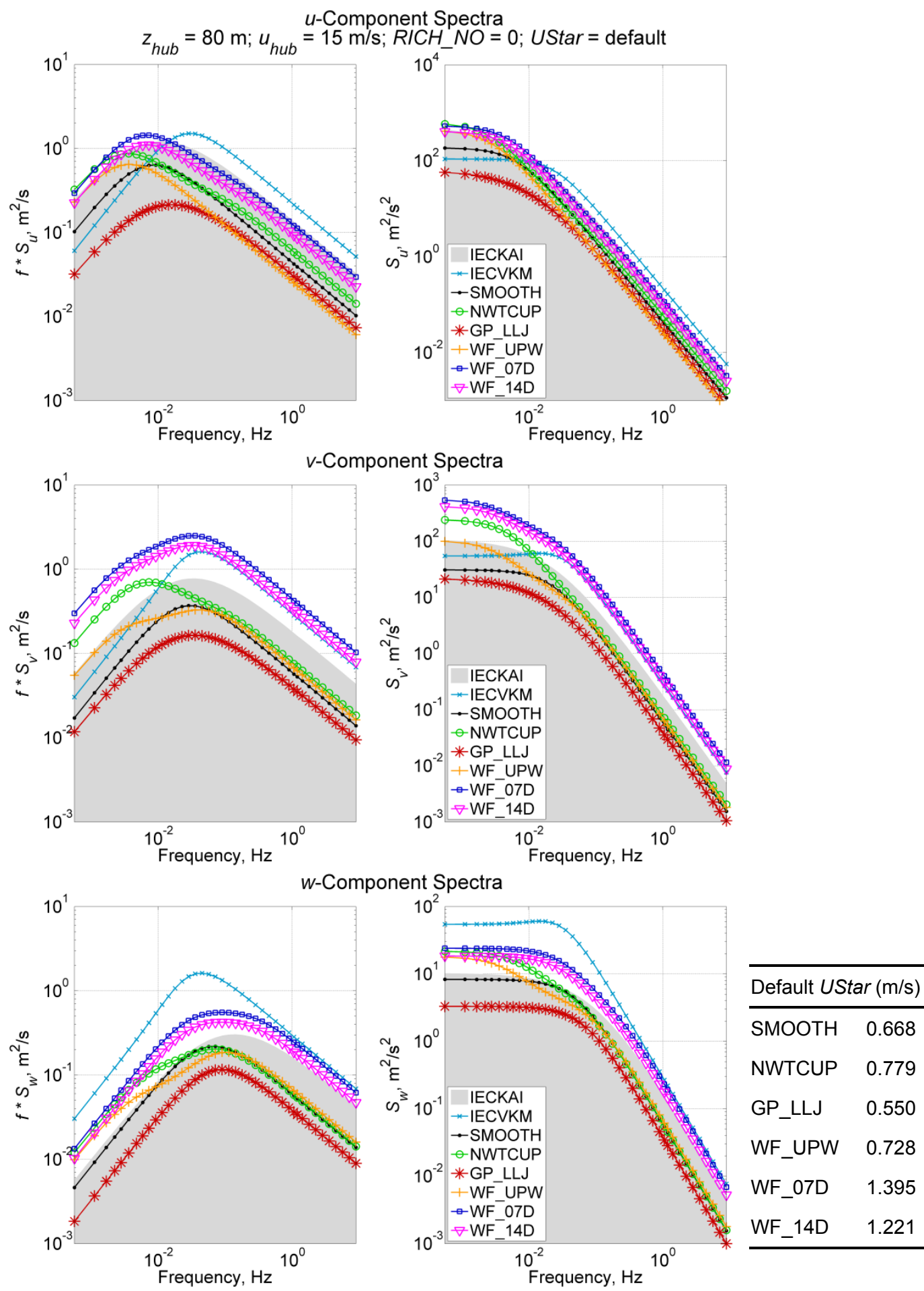

Figure G-1. Neutral velocity spectra for the 8 spectral models available in TurbSim, using a $15 \mathrm{~m} / \mathrm{s}$ wind speed at $80 \mathrm{~m}$; IECKAI and IECVKM use NTM category "B" and 61400-1 $3^{\text {rd }}$ ed. scaling; the non-IEC models use RICH_NO = 0 and UStar = "default" 

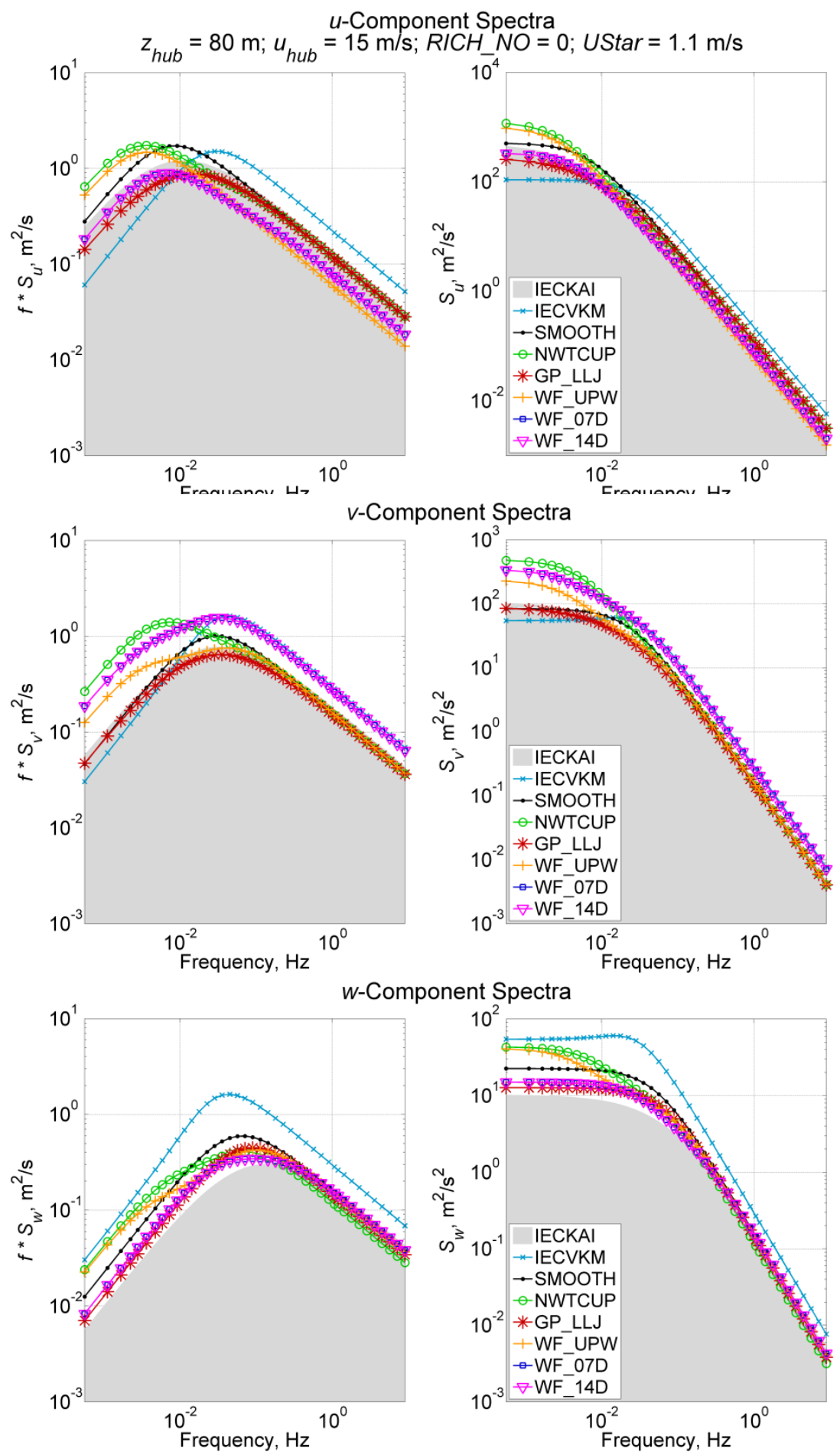

Figure G-2. Neutral velocity spectra for the 8 spectral models available in TurbSim, using a $15 \mathrm{~m} / \mathrm{s}$ wind speed at $80 \mathrm{~m}$; IECKAI and IECVKM use NTM category "B" and 61400-1 $3^{\text {rd }}$ ed. scaling; the non-IEC models use RICH_NO $=0$ and UStar $=1.1 \mathrm{~m} / \mathrm{s}$ 

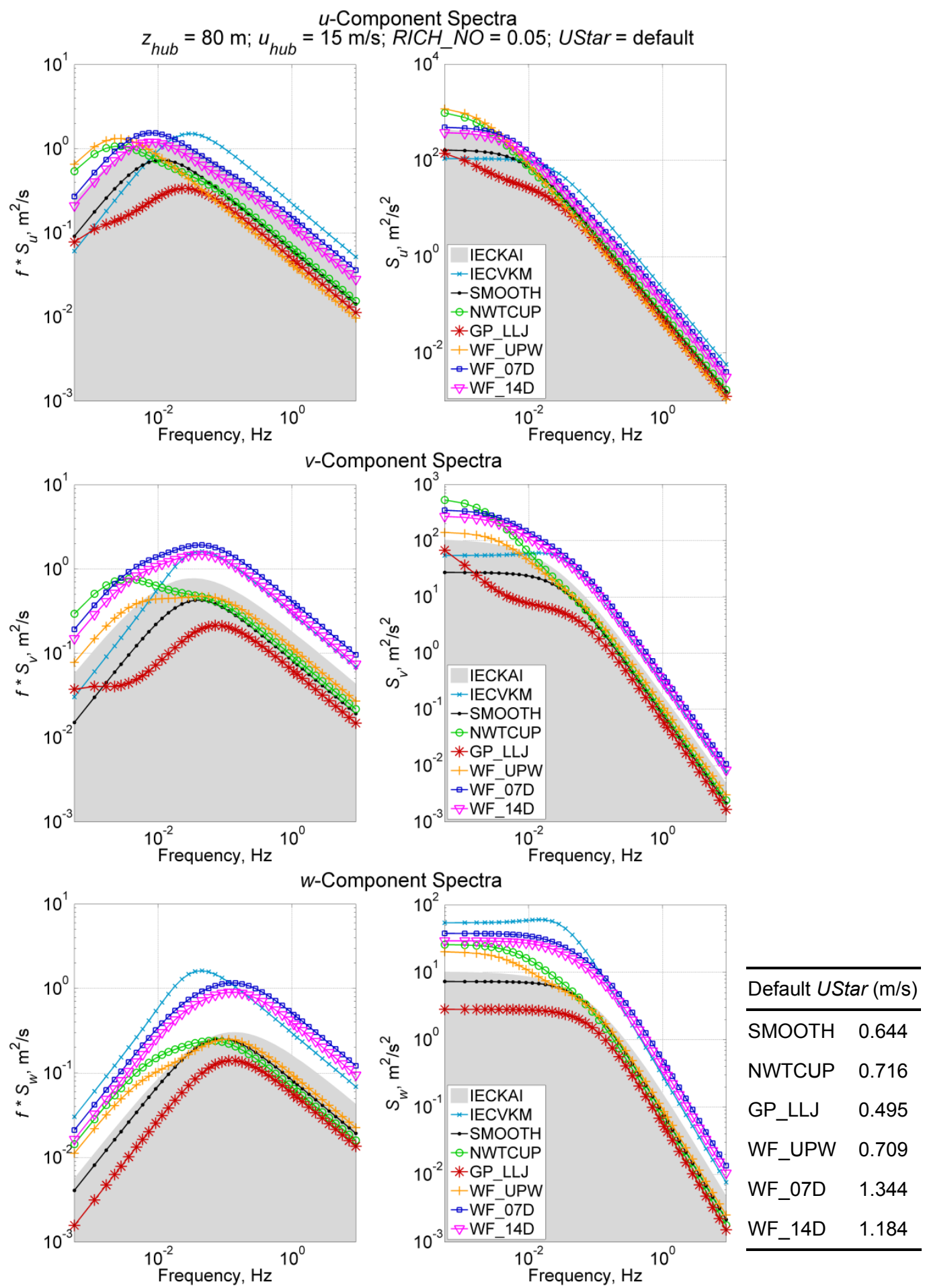

Figure G-3. Stable velocity spectra using a $15 \mathrm{~m} / \mathrm{s}$ wind speed at $80 \mathrm{~m}$; the non-IEC models use $R I C H \_N O=0.05$ and UStar = "default"; The IEC models, which are neutral $\left(R I C H \_N O=0\right)$, were added for reference; they use NTM category "B" and 61400-1 $3^{\text {rd }}$ ed. scaling 

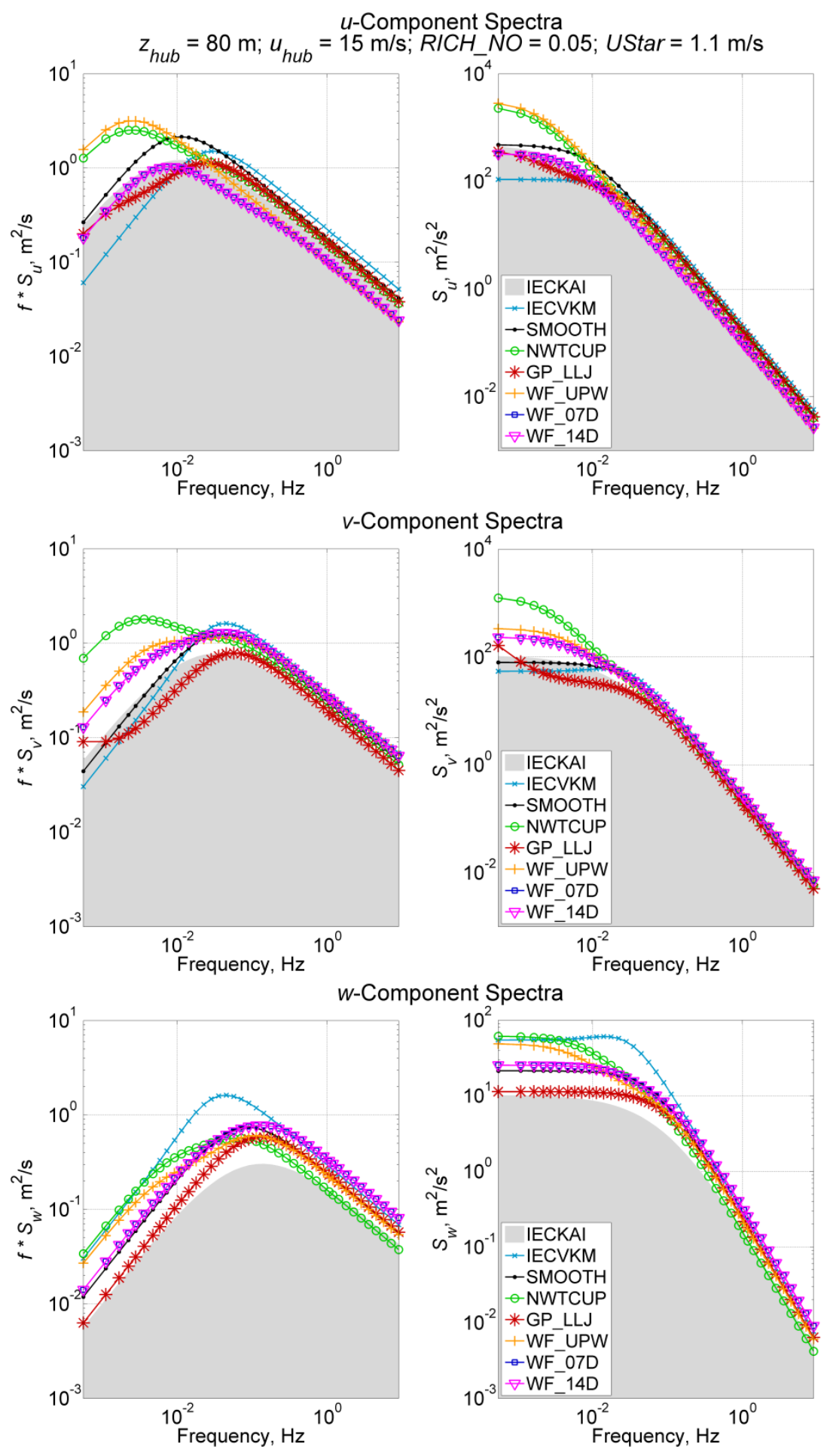

Figure G-4. Stable velocity spectra using a $15 \mathrm{~m} / \mathrm{s}$ wind speed at $80 \mathrm{~m}$; the non-IEC models use $\mathrm{RICH} \_\mathrm{NO}=0.05$ and UStar $=1.1 \mathrm{~m} / \mathrm{s}$; the IEC models, which are neutral $(\mathrm{RICH}$ _NO $=0$ ), were added for reference; they use NTM category "B" and 61400-1 $3^{\text {rd }}$ ed. scaling 

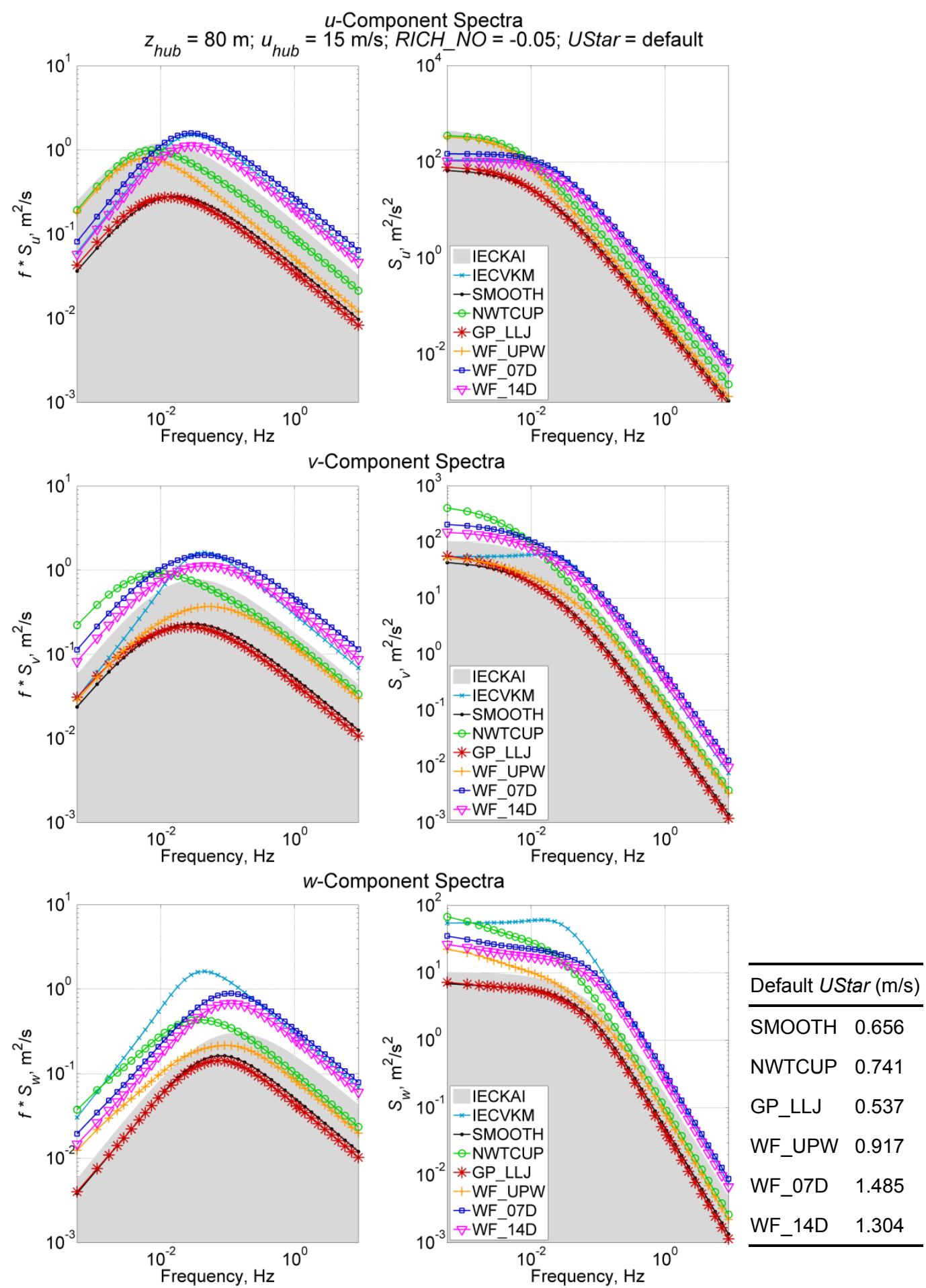

Figure G-5. Unstable velocity spectra using a $15 \mathrm{~m} / \mathrm{s}$ wind speed at $80 \mathrm{~m}$; the non-IEC models use $R I C H \_N O=-0.05$ and UStar = "default"; the IEC models, which are neutral $\left(R I C H \_N O=0\right)$, were added for reference; they use NTM category "B" and 61400-1 $3^{\text {rd }}$ ed. scaling 

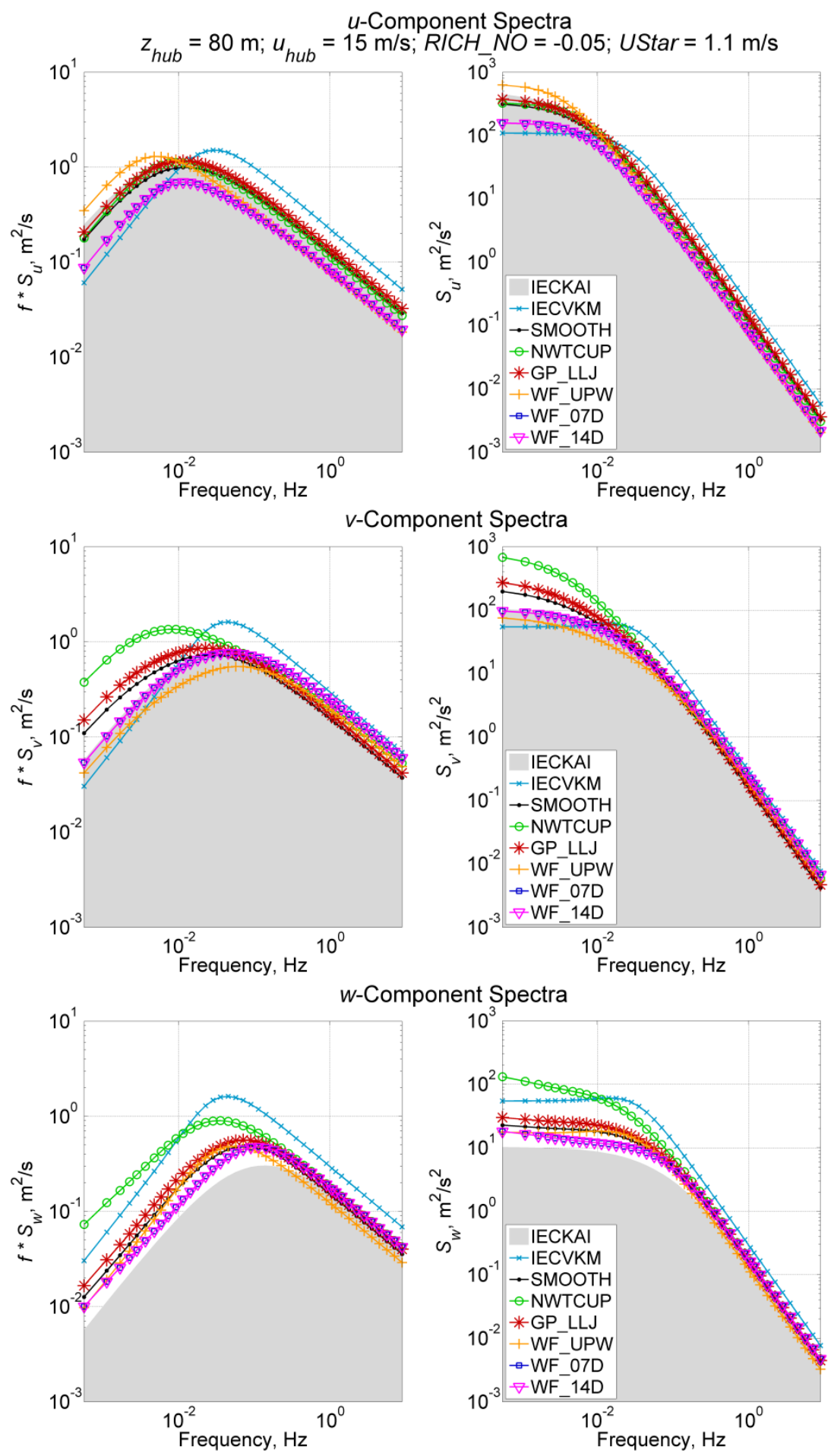

Figure G-6. Unstable velocity spectra using a $15 \mathrm{~m} / \mathrm{s}$ wind speed at $80 \mathrm{~m}$; the non-IEC models use $R I C H \_N O=-0.05$ and UStar $=1.1 \mathrm{~m} / \mathrm{s}$; the IEC models, which are neutral $\left(R I C H \_N O=0\right)$, were added for reference; they use NTM category "B" and 61400-1 $3^{\text {rd }}$ ed. scaling 


\section{Appendix H: Sample AeroDyn Coherent Turbulence Parameter Input} File

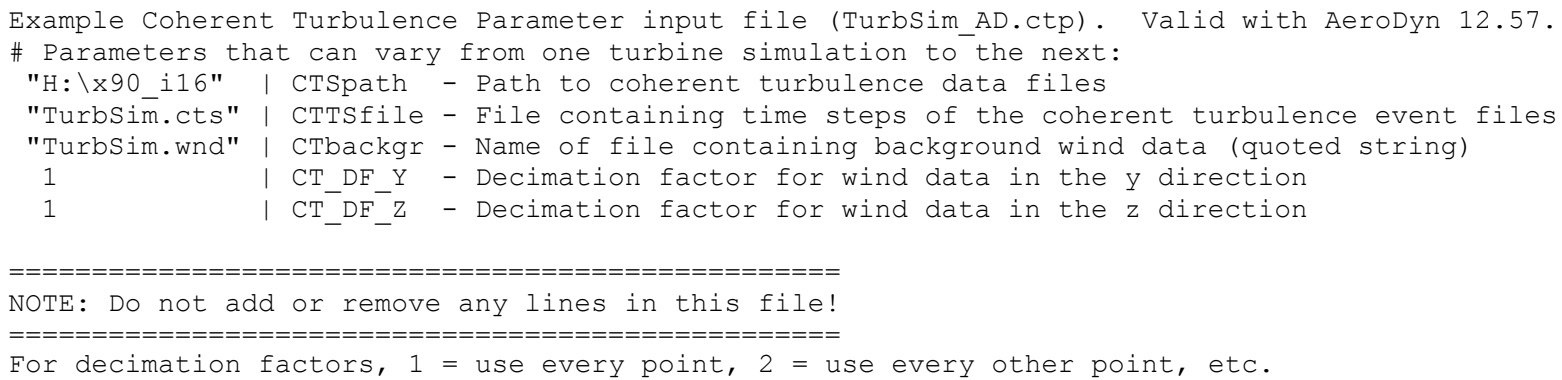

Figure H-1. Sample AeroDyn coherent turbulence parameter input file 


\section{REPORT DOCUMENTATION PAGE}

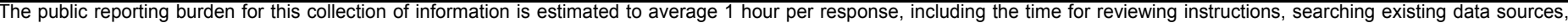

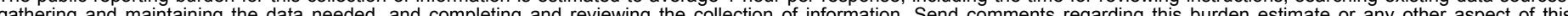

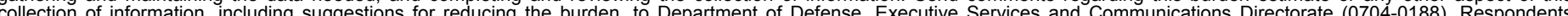

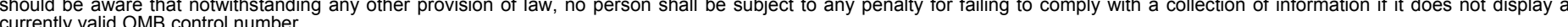

PLEASE DO NOT RETURN YOUR FORM TO THE ABOVE ORGANIZATION.
1. REPORT DATE (DD-MM-YYYY)
2. REPORT TYPE
3. DATES COVERED (From - To)

September 2009

Technical Report

4. TITLE AND SUBTITLE

TurbSim User's Guide: Version 1.50

5a. CONTRACT NUMBER

DE-AC36-08-GO28308

5b. GRANT NUMBER

5c. PROGRAM ELEMENT NUMBER

6. AUTHOR(S)

B.J. Jonkman

5d. PROJECT NUMBER

NREL/TP-500-46198

5e. TASK NUMBER

WER8.2102

5f. WORK UNIT NUMBER

7. PERFORMING ORGANIZATION NAME(S) AND ADDRESS(ES)

National Renewable Energy Laboratory

1617 Cole Blvd. REPORT NUMBER

Golden, CO 80401-3393

NREL/TP-500-46198

9. SPONSORING/MONITORING AGENCY NAME(S) AND ADDRESS(ES)

10. SPONSOR/MONITOR'S ACRONYM(S)

NREL

11. SPONSORING/MONITORING AGENCY REPORT NUMBER

12. DISTRIBUTION AVAILABILITY STATEMENT

National Technical Information Service

U.S. Department of Commerce

5285 Port Royal Road

Springfield, VA 22161

13. SUPPLEMENTARY NOTES

14. ABSTRACT (Maximum 200 Words)

The TurbSim stochastic inflow turbulence code was developed to provide a numerical simulation of a full-field flow that contains coherent turbulence structures that reflect the proper spatiotemporal turbulent velocity field relationships seen in instabilities associated with nocturnal boundary layer flows. This report provides a user's guide for the TurbSim code.

15. SUBJECT TERMS

wind turbine design code simulations; inflow turbulence code; TurbSim

\begin{tabular}{|c|c|c|}
\hline $\begin{array}{l}\text { a. REPORT } \\
\text { Unclassified }\end{array}$ & $\begin{array}{l}\text { b. ABSTRACT } \\
\text { Unclassified }\end{array}$ & $\begin{array}{l}\text { c. THIS PAGE } \\
\text { Unclassified }\end{array}$ \\
\hline
\end{tabular}

\begin{tabular}{l|l} 
17. LIMITATION & 18. \\
OF ABSTRACT & OF PAGES \\
UL &
\end{tabular}

19a. NAME OF RESPONSIBLE PERSON

19b. TELEPHONE NUMBER (Include area code) 